Article

\title{
Laser-Assisted (e, 2e) Collisions in the Symmetric/Asymmetric Coplanar Geometry
}

\author{
Abdelkader Makhoute ${ }^{1,2, *}$, Driss Khalil $^{1}$ and Imane Ajana ${ }^{1}$ (i) \\ 1 Physics of Radiation and Laser-Matter Interactions, Faculty of Sciences, Moulay Ismail University, Zitoune, \\ Meknes B.P. 11201, Morocco; drisskhalil@hotmail.com (D.K.); ajanaimane@gmail.com (I.A.) \\ 2 Faculty of Sciences, University Libre de Bruxelles (ULB), Boulevard du Triomphe, 1050 Bruxelles, Belgium \\ * Correspondence: makhoute@netcourrier.com
}

Received: 24 January 2019; Accepted: 9 March 2019; Published: 2 April 2019

check for updates

\begin{abstract}
In this review, we present a comprehensive survey of laser-assisted (e, 2e) reactions. The influence of a laser field on the dynamics of $(e, 2 e)$ collisions in atomic hydrogen is analyzed in the symmetric and asymmetric coplanar geometries. Particular attention is devoted to the construction of the dressed (laser-modified) target wave functions, in both the initial and final states. The calculation is performed in the framework of Coulomb-Volkov-Born approximation, where the initial and final electrons are described by Volkov wave functions, while the interaction of the incident electron with the target atom is treated in the first and the second Born approximation. The state of the ejected electron is described by a Volkov/Coulomb-Volkov wave function. A detailed account is also given of the techniques we have used to evaluate the scattering amplitudes. The influence of the laser parameters (frequency, intensity, and direction of polarization) on the angular distribution of the ejected electron is discussed, and a number of illustrative examples are given. The structure of the triple differential cross section in the vicinity of resonances is also analyzed.
\end{abstract}

Keywords: (e, 2e) collisions; symmetric and asymmetric coplanar geometries; Coulomb-Volkov-Born approximation; laser parameters; triple differential cross section

\section{Introduction}

The laser-assisted electron-impact scattering is currently drawing increasing attention from both experimental and theoretical researchers. From a practical point of view, the study of these processes finds potential importance in different applied areas, e.g., plasma confinement in fusion plasma, laser heating of plasmas [1,2], high-power gas lasers. Intense monochromatic laser fields have not only vastly increased spectroscopic resolution but also made possible the observation of multiphoton transitions that are essentially unobservable with conventional light sources. In view of the recent availability of increasingly more powerful and tunable lasers, laser-assisted elastic [3-8], and excitation processes [9-12], are nowadays being observed in laboratories.

The electron-impact single ionization, called (e, 2e) process, has become a powerful tool to investigate the dynamics of the ionization process. The study of such processes provides important information about target structure, target wave function, and collision dynamics. Since the pioneering (e, 2e) experiment carried out by Ehrhardt et al. [13], many (e, 2e) coincidence experiments were performed for various atomic targets and for a wide range of kinematic parameters [13-19]. Several studies were carried out to measure and calculate the triple differential cross sections (TDCS) for this process, which provide the most detailed information presently available about $(\mathrm{e}, 2 \mathrm{e})$ reactions. The rapid development in laser technology combined with the impressive advances in multiparticle detection techniques allowed the realization of the first kinematically complete experiment for the laser-assisted electron-impact ionization $[20,21]$. The experiment was performed using multiparticle 
imaging techniques "reaction microscopes" by overlapping a $1-k e V$ pulsed electron beam and a Nd:YAG laser beam at the position of a supersonic helium target beam. The experimental results are compared to the predictions of a first-order Born approximation calculation and clear deviations are observed.

The measurements of triply differential electron-impact ionization cross sections have revealed the presence of two different groups of electrons ejected during the ionization process. One of these, known as the "binary encounter" group, due to an encounter between the incoming and the atomic electron and is essentially independent of the nucleus. In this case the scattered incident electron and the ejected electron emerge on opposite sides of the incident electron direction. The second group has been called the "recoil" group and is thought to be due to electrons which have been initially scattered in the direction of the "binary encounter" group but which have subsequently suffered a reflection due to the attractive potential of the ion. They therefore emerge from the collision in the opposite direction to the binary encounter group. The physical picture for the generation of a slow electron in the angular range of the recoil peak is that several strong interactions take place in the collision complex or/and in the close neighborhood, namely (i) the electron-electron interaction of the fast incoming electron and one of the atomic electrons, (ii) the interaction of the slow ionization electron with the remaining ion, accompanied with relatively large momentum transfer to the ion, and (iii) final state interactions of the slow electron with the scattered electron and the ion.

It is useful when studying (e, 2e) collisions to distinguish between two kinematical arrangements, since these have important implications for the theoretical analysis of the collision. One is the asymmetric kinematics in which most of the (e, 2e) collisions events occur and the availability of most theoretical calculations and experimental measures for the corresponding field-free $(e, 2 e)$ reactions. The other one is symmetric kinematics which are defined by the requirement that the energies and angles of the scattered and ejected electrons are equal . However, for the last kinematic arrangement no laser-assisted $(\mathrm{e}, 2 \mathrm{e})$ experiment is yet available in the literature. These experiments are extremely difficult to perform, as the laser intensities that are required to produce a measurable effect are high, and so require a high power pulsed laser. Such laser systems operate at low repetition rate, and the laser pulse is only on for a short amount of time.

At an early stage of this research field, theoretical studies treated this process by neglecting the target dressing effects and described the unbound electrons either as non relativistic Volkov or Coulomb-Volkov states [22-28]. Later on, Joachain and coworkers investigated the case of hydrogen [29,30], and helium targets [31,32], while considering the dressing of the atomic states. They found that the dressing of the target by the laser field can significantly affect the TDCSs corresponding to ionizing processes. In these studies, the S-matrix elements were evaluated in the first Born approximation, which provides a useful tool for capturing the qualitative trends in the measured cross sections. It is usually assumed that the first Born approximation for electron-atom ionization becomes valid at sufficiently high impact energies [33].

A number of theoretical studies [24-26,29-32,34-36] were performed for hydrogen and helium atoms using different models in order to calculate TDCS at high initial energies. During the last few years, considerable progress has been achieved in the investigation of $(e, 2 e)$ phenomena at low and intermediate incident energy [37-39]. The Born approximation has been most widely used to calculate ionization cross sections. This model is flexible in analytical and numerical calculations and remains a preferable model to investigate the collision processes. At sufficiently high energies it is generally believed that the first Born approximation can be used to describe the direct ionization process [33]. It is therefore obviously necessary to extend the Born approximation by treating the projectile-target interaction up to second-order Born amplitude in the range of the low energy regime. In this review, we have treated the projectile-target interaction up to second-order Born amplitude. We have preformed second-order Born calculations of TDCS, in order to investigate the second-order effect in laser-assisted (e, 2e) reactions in the range of low incoming energies for the asymmetric coplanar geometry. This formalism is improved by the consideration of the exchange effect between the free outgoing electrons. Most work on the laser-assisted $(e, 2 e)$ reactions employed the first Born 
approximation due to the difficulty of the numerical calculation of the second Born term. In fact, the second Born approximation needs a difficult triple numerical integration [40] and often many authors find some controversial results. In the experiment of Höhr et al. [20], significant differences were observed (both in shape and magnitude) as compared to the field free situations which could not be explained in the first Born approximation. Thus the need for a more refined theory cannot be over emphasized, as was also pointed out by Hörr et al. [20]. This motivated us to attempt many works that study the laser-assisted single ionization of hydrogen and helium atoms in the Ehrhardt asymmetric geometry using a second-order Born calculation [41-46]. Our group have also studied the symmetric case by restricting to a first Born treatment $[47,48]$.

A number of theoretical works on laser-assisted (e, 2e) collisions in atomic systems have been published so far, and several approaches have been put forward. Most studies deal with the case of kinematics that involves small energy and momentum transfer $[49,50]$. Theoretical analyzes of laser-assisted electron-impact ionization at high impact energy and large momentum transfer have been delivered recently [51,52]. In the literature, it is often referred to as electron momentum spectroscopy (EMS). Experimentally, a new model describing the electron-impact ionization from laser-excited and aligned targets has yet been published [53]. Time-Resolved (e, 2e) Electron Momentum Spectroscopy has also been used for Molecular Orbital Imaging of the Acetone S2 by Yamaski Group [54]. Experimental results clearly demonstrate that EMS measurements of molecular excited states are feasible, opening the door to time-resolved orbital imaging in momentum space. Very recently, A new electron momentum spectroscopy apparatus, developed for time-resolved experiments in the nanosecond time scale, has been successfully constructed [55]. EMS measurements on argon in the pulsed electron beam mode have been reported, illustrating that the apparatus is ready to investigate the electronic structure of molecular excited states whose lifetime is nanosecond or longer.

In this review, we represent our results obtained of the triple differential cross sections in both the coplanar asymmetric and symmetric geometry. The calculation of the required radial amplitudes is performed by expanding the atomic wave functions onto a Sturmian basis, which allows us to exactly take into account the contribution of the continuous spectrum to the dressing of the atomic states. This method of computation constitutes an important advantage over the closure approximation used in the recent study of Zheng et al. [56]. The interaction of the laser field with the unbound electrons is treated in a nonperturbative way using Volkov wave functions [57]. We have used the first-order time-dependent perturbation theory to treat the influence of the laser field on the target states. The remainder of this paper is presented in seven sections. Section 2 presents a brief description of $(\mathrm{e}, 2 \mathrm{e})$ collision processes as well as the kinematics. Section 3 is devoted to present the wave functions and the techniques which we have used to evaluate the first and second-order Born TDCSs. In Sections 4 and 5, we present the theoretical treatment of laser-assisted (e, 2e) reactions in the coplanar asymmetric and symmetric geometries and discuss our numerical results, as well as their physical implications. Section 6 contains our conclusions. Unless otherwise stated, atomic units (a.u.) are used throughout.

\section{Description of (e, 2e) Collision Processes}

\subsection{Description}

Laser-assisted electron-atom collisions are interesting for several reasons. Firstly, from the experimental point of view, they allow the observation of multiphoton processes at relatively moderate laser intensities. Secondly, from the theoretical point of view, they require original methods for describing the influence of the laser field on the collisions. Indeed, the presence of the laser field introduces in the problem several new parameters (such as the laser frequency, intensity and polarization), the variation of which allows deeper insight into the fundamental processes to be obtained. Thirdly, laser-assisted electron-atom collisions are also important in several applied areas 
such as plasma heating by electromagnetic waves. Information on laser-assisted electron-atom collision processes is obtained by performing three-beam experiments, in which an atomic beam is crossed in coincidence by a laser beam and an electron beam, and the scattered electrons are detected. It is worth pointing out that the laser intensity should not be too high $\left(I<I_{a}\right)$, since otherwise the atom would be ionized. In the field of laser-assisted electron-atom interactions so far relatively little has been accomplished theoretically mainly due to the complexity of the problem, since, above all, there exists no exact solution for the problem of an electron moving simultaneously in a plane wave radiation field and in a Coulomb potential. A successful approach has been followed in the solution of the above problem; where the interaction of the scattered electron with the radiation field is treated exactly, while the interaction of the laser field with the atomic electrons is treated perturbatively. However, this approach requires that the local electric field strength $\mathcal{E}_{0}$ of the laser at the atomic site is much less than the atomic field strength $\mathcal{E}_{0} \ll e / a_{0}^{2}=5 \times 10^{9} \mathrm{~V} / \mathrm{cm}$ with $a_{0}$ being the Bohr-radius.

In this review, we shall concentrate on the most basic electron impact ionization process, namely the single step $(\mathrm{e}, 2 \mathrm{e})$ reaction in the presence of the laser field

$$
e^{-}+A(i)+\ell \omega \rightarrow A^{+}(f)+2 e^{-} .
$$

In order to keep calculational details to a minimum, our calculation will focus on the case of atomic hydrogen for which difficulties arising from the choice of the target wavefunctions and the motion of the ejected electron in the field of the ion are not present. Indeed, atomic hydrogen is ideal due to its analytically known wave functions, though this is a particularly difficult target for the experimentalists. As a consequence, no laser-assisted $(\mathrm{e}, 2 \mathrm{e})$ experiment is yet available in the literature for atomic hydrogen. Note, however, though simplified, the model contains all the ingredients needed for the discussion of the physics of such processes.

Let us consider a collision between a hydrogen target (in its ground state) and an electron moving along the $z$ axis in the presence of a laser field. As a result of the collision, the target atom becomes ionized and the projectile electron changes its momentum from $\mathbf{k}_{i}$ to $\mathbf{k}_{a}$. In the final state, two electrons emerge with momenta $\mathbf{k}_{a}$ and $\mathbf{k}_{b}$. This process can be described by the equation

$$
e^{-}\left(\mathbf{k}_{i}\right)+H(1 s)+\ell \omega \longrightarrow H^{+}+e^{-}\left(\mathbf{k}_{a}\right)+e^{-}\left(\mathbf{k}_{b}\right),
$$

The energy conservation equation corresponding to the laser-assisted (e, 2e) reaction of Equation (2) reads

$$
E_{k_{i}}+E_{0}+\ell \omega=E_{k_{a}}+E_{k_{b}},
$$

where $E_{0}=-0.5$ a.u. is the ground state energy of atomic hydrogen, while the atomic energy recoil is neglected. $E_{k_{i}}=k_{i}^{2} / 2, E_{k_{a}}=k_{a}^{2} / 2$ and $E_{k_{b}}=k_{b}^{2} / 2$ represent, respectively, the kinetic energy of the incident, scattered, and ejected electrons. The integer $\ell$ is the number of photons transferred between the projectile-target system and the laser field and $\omega$ is the laser frequency. Positive values of $\ell$ correspond to photon absorption (inverse bremsstrahlung), negative ones to photon emission (stimulated bremsstrahlung) and $\ell=0$ to a collision process in the laser field without net absorption or emission of photons.

\subsection{Kinematics}

It is useful when studying (e, 2e) coincidence experiments to distinguish between several kinematical arrangements, since these have important implications for the theoretical analysis of the collision, as we shall see later. A first distinction can be made between coplanar geometries such that the momenta $\mathbf{k}_{i}, \mathbf{k}_{a}$ and $\mathbf{k}_{b}$ are in the same plane as $\varphi_{a}=0, \varphi_{b}=0$ or $\pi$ and non-coplanar geometries such that the momentum $\mathbf{k}_{b}$ is out of the $\left(\mathbf{k}_{i}, \mathbf{k}_{a}\right)$ reference plane (see Figure 1). 


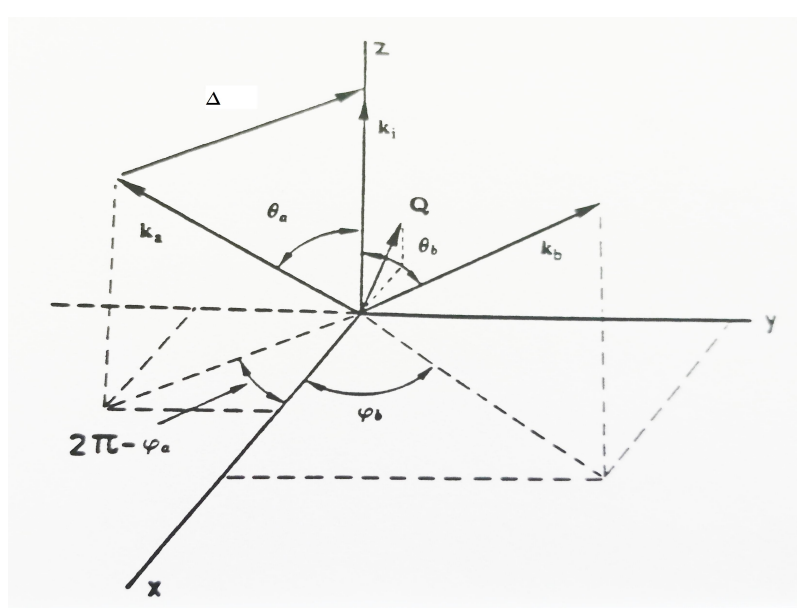

Figure 1. The kinematics of an $(e, 2 e)$ reaction. The incident electron is following the positive direction of the $z$ axis, with momenta $\mathbf{k}_{i}$. The momenta of the outgoing electrons are $\mathbf{k}_{a}$ and $\mathbf{k}_{b}$, respectively. Also shown are the angles $\theta_{a}$ and $\theta_{b}$ of the scattered and ejected electrons and $\Delta$ is the momentum transfer. When $\varphi_{a}-\varphi_{b}=0$ or $\varphi_{a}-\varphi_{b}=\pi$ the geometry is called coplanar.

Another useful distinction can be made between asymmetric and symmetric geometries. In asymmetric geometries, a fast electron of energy $E_{i}$ is incident on the target atom, and a fast ("scattered") electron $A$ is detected in coincidence with a slow ("ejected") electron B. Experiments of this kind were first performed by Ehrhardt et al. [13]. Since then a number of asymmetric (e, 2e) coincidence measurements have been carried out (see for example References [58-65]); they have been reviewed by Ehrhardt et al. [66]. In particular, the Kaiserslautern group of Ehrhardt and co-workers carried out extensive series of (e, 2e) asymmetric geometry experiments on atomic hydrogen, helium and other noble gases, in which they used intermediate energy electrons with typical energies of a few hundred $e V$. They selected a coplanar geometry and chose the scattering angle $\theta_{a}$ of the fast electron to be fixed and small, while the angle $\theta_{b}$ the slow electron was varied. It is worth noting that in this Ehrhardt-type asymmetric geometry the magnitude $\Delta$ of the momentum transfer $\Delta=\mathbf{k}_{i}-\mathbf{k}_{b}$ is small. In the asymmetric geometry, the energy of the electrons coming out of the reaction are well determined such that $E_{k_{a}} \gg E_{k_{b}}$ (see Figure 2a). The fast electron is scattered at an angle that is fixed and small $\left(\theta_{a}<10^{\circ}\right)$, while the ejection angle of the slow electron varies in the plane. Symmetric geometries are defined by the requirement that $\theta_{a} \simeq \theta_{b}$ and $E_{k_{a}} \simeq E_{k_{b}}$ (see Figure 2b). The first (e, 2e) symmetric coincidence experiments of Amaldi et al. [67] have been followed by a number of experiments of this type (see for example References [68-75]).

(a)

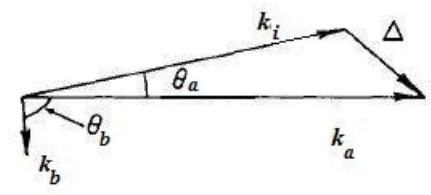

(b)

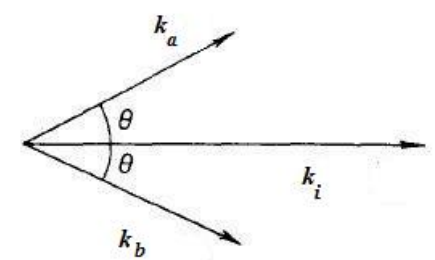

Figure 2. Schematic diagrams of the kinematics of (a) a coplanar asymmetric and (b) a coplanar symmetric (e, 2e) reaction. The vector $\Delta=\mathbf{k}_{i}-\mathbf{k}_{a}$ is the momentum transfer. In the symmetric case $\theta=\theta_{a}=\theta_{b}$

\subsection{Triple Differential Cross Sections}

The results of collision experiment are usually expressed in terms of characteristic quantities called cross sections. They are defined as follows: The cross section of a certain type of event in a given collision is the ratio of the number of events of this type per unit time and per unit scatterer, to the relative flux of the incident particles with respect to the target. 
Laser-assisted electron-impact ionization of atoms continues to attract considerable interest. In fact, the laser-assisted (e, 2e) collision of an atom enables experimental observations of different multiphoton processes at relatively moderate field intensities. It also allows the measurement of some electron-atom scattering parameters which otherwise (in the field free case) would not be accessible to experiments. Several studies were carried out to measure and calculate the triple differential cross sections (TDCS) for this process, which provide the most detailed information presently available about (e, 2e) reactions.

The most detailed information presently available about single ionization processes of the type Equation (1) has been obtained by analyzing triple differential cross sections (TDCS) measured in $(\mathrm{e}, 2 \mathrm{e})$ coincidence experiments. Indeed, the measurable quantity which is calculated by theory is so called triplet differential cross sections, i.e., the coincident differential cross section that depends on the energies and the emission solid angles of the continuum electrons. The TDCS is a measure of the probability that in an $(\mathrm{e}, 2 \mathrm{e})$ reaction an incident electron of momentum $\mathbf{k}_{i}$ and energy $E_{k_{i}}$ will produce on collision with the target two electrons having energies $E_{k_{a}}$ and $E_{k_{b}}$ and momenta $\mathbf{k}_{a}$ and $\mathbf{k}_{b}$, emitted respectively into the solid angles $d \Omega_{a}$ and $d \Omega_{b}$ centered about the directions $\left(\theta_{a}, \phi_{a}\right)$ and $\left(\theta_{b}, \phi_{b}\right)$.The TDCS is usually denoted by the symbol $d^{3} \sigma / d \Omega_{a} d \Omega_{b} d E_{b}$. By integrating the TDCS over $d \Omega_{a}, d \Omega_{b}$ or $d E_{b}$ one can form various double and single differential cross sections. Finally, the total ionization cross section is obtained by integrating over all outgoing electron scattering angles and energies, and depends only on $E_{0}$, the incident electron energy. It has been found that when few multiphoton processes are involved, the qualitative behavior of the cross sections as a function of the solid angles of the final state electrons and the electron energies are modified markedly by the presence of the laser field. The cross sections were also found to depend decisively on the dressing of the atomic target states.

\section{Theory of (e, 2e) Collisions in the Presence of a Laser Field}

\subsection{Description of the Laser Field}

We shall discuss the theory of the laser-atom interactions based on a semi-classical approach which provides the framework for studying the atomic multiphoton processes in the presence of a laser field. In particular, we shall introduce the dipole approximation, in which the laser field is described by a spatially homogeneous electric-field component, while its magnetic-field component vanishes. The dipole approximation is fully adequate to investigate atomic multiphoton processes over a wide range of laser frequencies and intensities. However, as the intensity increases beyond critical values that depend on the frequency, non-dipole effects due to the magnetic-field component of the laser field, and eventually relativistic effects, must be taken into account.

We assume that the wavelength $\lambda$ of the laser field is large compared with the size of the atomic system under consideration and that the laser field intensity is not too high. When these two conditions are fulfilled, the dipole approximation can be made, which consists in neglecting the spatial variation of the radiation field across the atom. In this approximation, for an atom whose nucleus is located at the position $r_{0}$, the vector potential $A\left(r_{0}, t\right)=A(t)$ is spatially homogeneous (i.e., it depends only on the time variable).

We have considered in our calculations that the collision dynamics is treated quantum mechanically, while the laser field is treated classically, and is chosen to be single mode, and spatially homogenous electric field. Working in the Coulomb gauge we have for the vector potential of a field propagating along the $\hat{z}$ axis and represented in the collision plane $(\hat{x}, \hat{y})$

$$
A(t)=A_{0}\left(\hat{x} \cos (\omega t+\varphi)+\hat{y} \sin (\omega t+\varphi) \tan \left(\frac{\eta}{2}\right)\right)
$$


The corresponding electric field is

$$
\mathcal{E}(t)(t)=\mathcal{E}_{0}\left(\hat{x} \sin (\omega t+\varphi)-\hat{y} \cos (\omega t+\varphi) \tan \left(\frac{\eta}{2}\right)\right),
$$

where $\eta$ measures the degree of ellipticity of the field such as $(\eta=0)$ corresponds to a linear polarization and $\left(\eta=\frac{\pi}{2}\right)$ to a circular polarization. $\mathcal{E}_{0}=\omega A_{0} / c, \mathcal{E}_{0}$ and $\omega$ are the peak electric field strength and the laser angular frequency, respectively. Here $\varphi$ denotes the initial phase of the laser field. We can recast the electric laser field in terms of its spherical components by

$$
\mathcal{E}(t)=\mathcal{E}_{0} \sum_{\nu= \pm 1} i v \hat{\varepsilon} \exp (-i v(\omega t+\varphi))
$$

where $\hat{\varepsilon}=\frac{1}{2}\left[\hat{\mathbf{x}}+i v \hat{\mathbf{y}} \tan \left(\frac{\eta}{2}\right)\right]$ is the unitary polarization vector.

The dynamics of the scattering processes can be strongly modified if it takes place in the presence of a laser field. In fact, the laser usually plays the role of a third body and introduces some new degrees of freedom (e.g., the intensity, the frequency, and the polarization). Thus, it might be possible to control the collision process by a suitable choice of laser parameters as well as the projectile energy. Further, the addition of a laser field leads to essential modifications (dressing) of the target states and it also influences decisively the state of the continuum electrons. The laser-assisted differential cross sections could differ strongly in shape and magnitude if the dressing of the atomic target by the laser field is taken into account.

\subsection{The Wave Function of the Free Electron in the Presence of a Laser Field}

We begin by obtaining the wavefunction of a free electron in the presence of a laser field. The non-relativistic motion of this electron is described by the Schrodinger equation

$$
i \frac{\partial}{\partial t} \psi(\boldsymbol{r}, t)=H_{L} \psi(\boldsymbol{r}, t)=\frac{1}{2}\left(\boldsymbol{p}+\frac{A(t)}{c}\right)^{2} \psi(\boldsymbol{r}, t),
$$

which is readily solved to give the Volkov wavefunction [57]

$$
\chi_{k}(\boldsymbol{r}, t)=(2 \pi)^{-\frac{3}{2}} \exp \left\{i\left(\boldsymbol{k} \cdot \boldsymbol{r}-E_{k} t-R_{k} \sin \left(\omega t-\gamma_{k}\right)\right)\right\},
$$

where

$$
R_{k}=\alpha_{0}\left((k \cdot \hat{x})^{2}+(k \cdot \hat{y})^{2} \tan ^{2}(\eta / 2)\right)
$$

and

$$
\tan \left(\gamma_{k}\right)=\frac{k \cdot \hat{y}}{k \cdot \hat{x}} \tan \left(\frac{\eta}{2}\right),
$$

where $\alpha_{0}=\frac{\mathcal{E}_{0}}{\omega^{2}}$ being the amplitude associated to the classical quiver motion of the electron in the laser field, $k$ denotes the electron wavevector and $E_{k}=\frac{k^{2}}{2}$ is its kinetic energy. In writing the Volkov solution Equation (8) we have omitted the $A^{2}$ term which can be removed from the Schrodinger Equation (7) by a unitary transformation. The Volkov wave function of Equation (8) is normalized to a $\delta$ function.

\subsection{The Dressed Target Wave Function in the Initial State}

The dressed states of the target atom embedded in the laser field are obtained by solving the Schrödinger equation

$$
i \hbar \frac{\partial}{\partial t} \Phi\left(q_{1}, q_{2}, \ldots, q_{Z}, t\right)=H_{T} \Phi\left(q_{1}, q_{2}, \ldots, q_{Z}, t\right),
$$


where $Z$ is the atomic number of the atom, $q_{i} \equiv\left(\mathbf{r}_{i}, \sigma_{i}\right)$ denotes the ensemble of space and spin coordinates of the $i$ th target electron and $H_{T}$ is the hamiltonian of the target atom in the presence of the laser field. In the following, we shall restrict our attention to laser-assisted collision from the ground state of light atoms such as hydrogen and helium where spin effects can be neglected. Moreover, we shall consider laser fields which, although strong by laboratory standards, are nevertheless less than the atomic unit of field strength such that $\mathcal{E}_{0} \ll e / a_{0}^{2}$, where $e / a_{0}^{2} \simeq 5 \times 10^{9} \mathrm{~V} \mathrm{~cm}^{-1}$ is the atomic unit of field strength (corresponding to an intensity $I_{0}=3.5 \times 10^{16} \mathrm{~W} \mathrm{~cm}^{-2}$ ). The method also applies to all laser frequencies, except of course in those case in which the laser photon energy is close to the energy of an atomic transition (vicinity of a bohr frequency), further limitations are imposed on the laser intensity, to first-order in the electric field corresponding to the laser field and the velocity gauge, the dressed wave functions corresponding to the initial bound state are given for an elliptically polarized field by [76]

$$
\phi_{0}(\boldsymbol{r}, t)=e^{-i \boldsymbol{a} \cdot \boldsymbol{r}} e^{-i E_{0} t}\left\{\psi_{0}(\boldsymbol{r})+\frac{i}{2} \sum_{n}\left(\frac{e^{i \omega t} M_{n 0}^{-}}{\omega_{n 0}+\omega}-\frac{e^{-i \omega t} M_{n 0}^{+}}{\omega_{n 0}-\omega}\right) \psi_{n^{\prime}}(\boldsymbol{r})\right\},
$$

where $I=A / c$, where $e^{-i a . r}$ is a gauge factor. $\psi_{n}$ is a target state of energy $E_{n}$ in the absence of the laser field, $\omega_{n 0}=E_{n}-E_{0}$ is the Bohr frequency, and $M_{n 0}^{ \pm}=\mathcal{E}_{0}\left\langle\psi_{n}\left|\hat{\varepsilon}_{ \pm} . r\right| \psi_{0}\right\rangle$ is the dipole coupling matrix elements.

\subsection{The Dressed Target Wave Function in the Final State}

Finding an adequate expression of the dressed continuum wave function $\phi_{k_{b}}\left(\boldsymbol{r}_{1}, t\right)$ is, in general, a more complicated task. In the case of sufficiently low laser intensities or high laser frequencies, one can expect that the coupling of the slow electron with the external field will be much weaker than the Coulomb interaction, so that a perturbative treatment of the laser field can still be used. In this case, the positive-energy solutions of the Schrodinger equation

$$
i \frac{\partial}{\partial t} \phi_{k_{b}}(\boldsymbol{r}, t)=\left[H_{0}+c^{-1} \boldsymbol{A}(t) \cdot \boldsymbol{p}\right] \phi_{k_{b}}(\boldsymbol{r}, t)
$$

where $\boldsymbol{p}=-i \nabla_{r}$ and $H_{0}=p^{2} / 2-1 / r$ is the hydrogen atom Hamiltonian and can be obtained to first order in the external field in a form analogous to Equation (12). That is,

$$
\begin{aligned}
\phi_{k_{b}}(\boldsymbol{r}, t) & =e^{-i \boldsymbol{a} \cdot \boldsymbol{r}} e^{-i E_{k_{b}} t}\left\{\psi_{c, k_{b}}^{(-)}(\boldsymbol{i})\right. \\
& \left.+\frac{i}{2} \sum_{n}\left(\frac{e^{i \omega t}}{E_{n}-E_{k_{b}}+\omega}-\frac{e^{-i \omega t}}{E_{n}-E_{k_{b}}-\omega}\right) M_{n k_{b}} \psi_{n}(\boldsymbol{r})\right\}
\end{aligned}
$$

where $\psi_{c, k_{b}}^{(-)}$is a Coulomb wave function with incoming wave behavior, corresponding to momentum $\boldsymbol{k}_{b}$ and normalized to a $\delta$ function in momentum space

$$
\psi_{c, k_{b}}^{(-)}(\boldsymbol{r})=(2 \pi)^{-3 / 2} e^{\pi / 2 k_{b}} e^{i k_{b} \cdot r} \Gamma\left(1+i / k_{b}\right){ }_{1} F_{1}\left[-i / k_{b}, 1,-i\left(k_{b} r_{1}+k_{b} \cdot r\right)\right] .
$$

In Equation (14) we have also introduced the dipole-coupling matrix elements $M_{n k_{b}}=\left\langle\psi_{n}\right|$ $\mathcal{E}_{0} \cdot \boldsymbol{k}\left|\psi_{c, k_{b}}^{(-)}\right\rangle$.

Let us now turn to the case of high laboratory intensities (with still $\mathcal{E} \ll e / a_{0}^{2}$ ) and low laser frequencies. In this instance, the perturbative result given by Equation (14) should be modified in order to account for the strong coupling which is characteristic of the interaction between an unbound particle and an intense external field in the infrared limit. For this purpose, let us substitute in Equation (13) the ansatz proposed by Banerji and Mittleman [24] 


$$
\phi_{k_{b}}(\boldsymbol{r}, t)=\exp \left(-i \boldsymbol{k}_{b} \cdot \boldsymbol{\alpha}_{0} \sin (\omega t)\right) \vartheta_{k_{b}}^{(-)}(\boldsymbol{r}, t) .
$$

We then obtain for the unknown function $\vartheta_{k_{b}}^{(-)}\left(\boldsymbol{r}_{1}, t\right)$ the equation

$$
i\left[\frac{\partial}{\partial t}-H_{0}\right] \vartheta_{k_{b}}^{(-)}(\boldsymbol{r}, t)=\omega \alpha_{0} \cdot\left(\boldsymbol{p}-\boldsymbol{k}_{b}\right) \cos (\omega t) \vartheta_{k_{b}}^{(-)}(\boldsymbol{r}, t),
$$

The advantage of this equation with respect to the original Equation (13) is to allow for a perturbative treatment of the right-hand side under three distinct conditions.

1. In the case of weak fields, such that $\alpha_{0} \ll 1$. This case has already been discussed above, and we have seen that an appropriate dressed wave function is then given by Equation (14).

2. In the limit of low frequencies.

3. For sufficiently high electron energies. Indeed, in this case the wave function describing an electron in the field of the residual ion is well approximated by the plane wave $\exp \left(i k_{b} \cdot r\right)$, and the operator $\left(\boldsymbol{p}-\boldsymbol{k}_{b}\right)$ gives zero when acting on this plane wave.

If, as a first approximation, we completely neglect the right-hand side of Equation (17), we obtain, by using Equation (16), the zeroth-order result

$$
\phi_{k_{b}}(\boldsymbol{r}, t) \simeq \exp \left(-i E_{k_{b}} t\right) \exp \left(-i \boldsymbol{k}_{b} \cdot \boldsymbol{\alpha}_{0} \sin (\omega t)\right) \psi_{c, k_{b}}^{(-)}(\boldsymbol{r}) .
$$

It amounts to approximate the wave function of the electron embedded in the field of the residual ion and the laser by the wave function describing the electron in the field of the ion alone, modulated in time by the same phase factor $\exp \left(-i k_{b} \cdot \alpha_{0} \sin (\omega t)\right)$ as the one multiplying the plane wave in the Volkov solution Equation (8). The approximate solution Equation (18) presents the advantage of containing the field to all orders; however, it completely decouples the electron-laser and electron-proton interactions.

Further progress may be achieved by solving Equation (17) using first-order time-dependent perturbation theory. By working in the momentum gauge, it is found using Equations (16) and (17) that

$$
\begin{aligned}
\phi_{k_{b}}(\boldsymbol{r}, t) & =e^{-i E_{k_{b}}{ }^{t}} e^{-i \boldsymbol{k}_{b} \cdot \boldsymbol{\alpha}_{0} \sin (\omega t)}\left[\psi_{c, k_{b}}^{(-)}(\boldsymbol{r})\right. \\
& -\frac{1}{2 \omega} \sum_{n}\left(\frac{e^{i \omega t}}{E_{n}-E_{k_{b}}+\omega}+\frac{e^{-i \omega t}}{E_{n}-E_{k_{b}}-\omega}\right) \\
& \left.\times\left\langle\psi_{n}\left|\mathcal{E}_{0} \cdot\left(\boldsymbol{p}-\boldsymbol{k}_{b}\right)\right| \psi_{c, k_{b}}^{(-)}\right\rangle \psi_{n}(\boldsymbol{r})\right] .
\end{aligned}
$$

On the other hand, if one first performs the gauge transformation

$$
\phi_{k_{b}}(\boldsymbol{r}, t)=\exp (-i \boldsymbol{a} . \boldsymbol{r}) \tilde{\phi}_{k_{b}}(\boldsymbol{r}, t)
$$

and then seeks a perturbative solution for the new wave function $\tilde{\phi}_{k_{b}}$, one obtains the expression

$$
\begin{aligned}
\phi_{k_{b}}(\boldsymbol{r}, t) & =e^{-i E_{k_{b}} t} e^{-i a \cdot r} e^{-i k_{b} \cdot \boldsymbol{\alpha}_{0} \sin (\omega t)}\left[\psi_{c, k_{b}}^{(-)}(\boldsymbol{r})\left(1+i \boldsymbol{k}_{b} \cdot \boldsymbol{\alpha}_{0} \sin (\omega t)\right)\right. \\
& \left.+\frac{i}{2} \sum_{n}\left(\frac{e^{i \omega t}}{E_{n}-E_{k_{b}}+\omega}-\frac{e^{-i \omega t}}{E_{n}-E_{k_{b}}-\omega}\right) M_{n k_{b}} \psi_{n}(\boldsymbol{r})\right]
\end{aligned}
$$

The results given by Equations (19) and (21) are readily seen to be equivalent up to first order in the perturbation $\omega \boldsymbol{\alpha}_{0} \cdot\left(\boldsymbol{p}-\boldsymbol{k}_{b}\right) \cos (\omega t)$. However, the expression (21) does not exhibit the spurious 
$\omega^{-1}$ divergence which is present in Equation (19). The result Equation (21) directly generalizes the perturbative expression (14). It differs from the expression obtained by Banerji and Mittleman [24], who made the additional approximation of taking the soft-photon limit in Equation (19). This last step is not justified because the dominant contribution to the sum over $\mathrm{n}$ arises from intermediate states with energies $E_{n} \simeq E_{k_{b}} \pm \omega$ or $E_{n}=E_{k_{b}}$.

It is worth noting that our result Equation (21) contains the laser field to all orders via the factor $\exp \left(-i \boldsymbol{k}_{b} \cdot \boldsymbol{\alpha}_{0} \sin (\omega t)\right)$, but in an approximate way which essentially describes the 'quasifree' behavior of the electron. On the other hand, the explicit role of the atomic states dressing the wave function is included to first order in $\mathcal{E}_{0}$.

\subsection{The Semi-Perturbative Method}

For a laser pulse which is not too intense, so that its peak electric-field strength is much smaller than the electric fields due to the Coulomb interactions experienced by the atomic electrons, the interaction of the atom with the laser field can be treated as a perturbation. The semi-perturbative theory of Byron and Joachain [77] is suitable for analyzing fast electron-atom collisions in the presence of relatively strong laser fields, and allows us to calculate how the "dressing" of the atomic states by the laser field can influence the collision cross sections. We shall now discuss the semi-perturbative theory of Byron and Joachain [77]. In fact, in semi-perturbative approaches, some of the interactions are treated in a non-perturbative way, and the remaining ones are treated by using perturbation theory.

The theoretical analysis of electron-atom processes implies that three types of interactions must be taken into account. The first one, which is also present in the absence of the laser field, is the interaction between the projectile electron and the target atom. The second one is the interaction between the laser field and the unbound electron(s). The third one is the interaction between the laser field and the target atom, which is responsible for the "dressing" of the atomic target states. In order to deal with this problem, Byron and Joachain have proposed a semi-perturbative theory. In this approach, the interaction of the laser field with the unbound electron(s) is treated exactly. On the other hand, the projectile electron-target atom interaction is treated perturbatively by using the Born series [78,79]. Finally, the interaction of the laser field with the target atom, responsible for target-dressing effects, can be treated perturbatively by using first-order time-dependent perturbation theory provided that (i) the electric-field strength remains small with respect to the atomic unit of electric field strength and (ii) the laser frequency is not close to resonance with an atomic transition frequency between the initial or final state of the atom and an atomic intermediate state. This approach allows to take into account target-dressing effects for laser fields having moderate intensities. The semi-perturbative theory discussed above has been applied to a variety of laser-assisted electron-atom processes: elastic [77], inelastic [80] collisions and $(e, 2 e)$ collisions $[29,30]$ in the presence of the laser field.

The semi-perturbative treatment of laser-assisted $(e, 2 e)$ reactions, described above for the case of an atomic hydrogen target, has been extended by Joachain et al. [29], Khalil et al. [31], Makhoute et al. [32,44,46], Bouzidi et al. [81] and Ajana et al. [43,45] to laser-assisted (e, 2e) reactions in helium, for which the possibilities of performing experiments are more favorable. These studies also showed that the choice of the laser parameters can strongly influence the dynamics of such processes, and that target-dressing effects can be important. The first experimental kinematically complete measurements of laser-assisted (e, 2e) reactions in helium, performed by Höhr et al. [20], confirmed the existence of distinct differences in TDCS between laser-on and laser-off conditions.

\section{Laser-Assisted (e, 2e) Collisions in Atomic Hydrogen in the Coplanar Asymmetric Geometry}

The experimental study of laser-assisted $(e, 2 e)$ collisions requires three coincident beams: an electron beam, an atomic beam and a laser beam. The incident electron beam is produced by an electron source in which the electrons are accelerated in an electric field that determines their energy. This electron beam must be well collimated and nearly monoenergetic, with a small spread (in direction and magnitude) of the incident electron momenta about the momentum $\boldsymbol{p}_{i}=\hbar \boldsymbol{k}_{i}$. The corresponding 
incident energy of the electrons is then $E_{k_{i}}=\hbar^{2} k_{i}^{2} /(2 m)$, with a small energy spread (or resolution) $\Delta E \ll E_{k_{i}}$. This energy spread is due partly to stray electrostatic fields penetrating the focusing lenses, and partly to space charges generated by the beam. The atomic target is a gaseous beam which must be of small size with uniform high density. The laser beam is produced by a laser whose pulse parameters (photon energy, intensity, polarization and duration) must be chosen carefully. Recently, Höhr et al. [20] have studied the electron impact single ionization of helium in the presence of a Nd:YAG laser pulse of wavelength $1064 \mathrm{~nm}$ easeand intensity $4 \times 10^{12} \mathrm{~W} \mathrm{~cm}^{-2}$. A $1 \mathrm{keV}$ pulse electron beam overlapped the laser beam at the position of a helium target beam. Höhr et al., observed distinct differences in the doubly and triply differential cross sections compared to the corresponding field-free cross sections. A detailed comparison between the experimental data and the theoretical calculations must take into account the actual experimental conditions, such as beam focusing and pulsed and possibly multi-mode operation of the laser. The spatial and temporal inhomogeneities of laser beams and their influence on laser-assisted electron-atom measurements have been investigated theoretically by Bivona et al. [82] and Francken and Joachain [83].

\subsection{Theory}

We assume the laser field to be classical, monochromatic, spatially homogeneous over atomic dimensions, and linearly polarized. Working in the Coulomb gauge, we have for the electric field $\mathcal{E}(t)=\mathcal{E}_{0} \sin (\omega t+\varphi)$ the corresponding vector potential $A(t)=A_{0} \cos (\omega t+\varphi)$ with $A_{0}=c \mathcal{E}_{0} / \omega$. Remembering that in the Ehrhardt asymmetric coplanar geometry, a fast electron of momentum $k_{i}$ is incident on the helium target, and a fast scattered electron of momentum $\boldsymbol{k}_{a}$ is detected in coincidence with a slow ejected electron of momentum $\boldsymbol{k}_{b}$, the three momenta $\boldsymbol{k}_{i}, \boldsymbol{k}_{a}$, and $\boldsymbol{k}_{b}$ being in the same plane. In addition, the scattering angle $\theta_{a}$ of the fast electron is fixed and small, while the angle $\theta_{b}$ of the slow electron is varied.

The central quantity to be evaluated is therefore the direct first Born S-matrix element [29]

$$
\mathbf{S}_{i o n}^{B_{1}}=-i \int_{-\infty}^{+\infty} d t\left\langle\chi_{k_{a}}\left(\boldsymbol{r}_{0}, t\right) \phi_{k_{b}}\left(\boldsymbol{r}_{1}, t\right)\left|V_{d}\left(\boldsymbol{r}_{0}, \boldsymbol{r}_{1}\right)\right| \chi_{k_{i}}\left(\boldsymbol{r}_{0}, t\right) \phi_{0}\left(\boldsymbol{r}_{1}, t\right)\right\rangle,
$$

with

$$
V_{d}\left(r_{0}, r_{1}\right)=-\frac{1}{r_{0}}+\frac{1}{r_{01}} .
$$

In this equation $r_{0}$ denotes the coordinate of the incident (and scattered) electron, $r_{1}$ is the coordinate of the target electron, and $r_{01}=\left|r_{0}-r_{1}\right|$. The wave functions $\chi_{k_{i}}$ and $\chi_{k_{a}}$ are Volkov wave functions describing, respectively, the motion of the incident and scattered electrons in the presence of the laser field. They are given by Equation (8).

The wave functions $\phi_{0}\left(\boldsymbol{r}_{1}, t\right)$ and $\phi_{k_{b}}\left(\boldsymbol{r}_{1}, t\right)$ appearing in Equation (22) are "dressed" states of the hydrogen atom embedded in the laser field. The first one corresponds to the initial bound state, and the second one to the final continuum state consisting of an ejected electron of momentum $\mathbf{k}_{b}$ moving under the combined influence of the proton and the laser field. For electric field strengths $\mathcal{E}_{0}$ small with respect to the atomic unit of field strength $\left(\mathcal{E}_{0} \ll \frac{e}{a_{0}^{2}} \simeq 5 \times 10^{11} \mathrm{~V} \cdot \mathrm{m}^{-1}\right)$, which is the case considered in this work, we can use the first-order time-dependant perturbation theory to obtain explicit expressions of the dressed atomic bound states. The dressed ground-state wave function of the hydrogen atom is given by Equation (12), while the dressed continuum wavefunction we have used is given by Equation (21).

Substituting the expressions (8), (12) and (21) into the first Born S-matrix element (22) and performing the time integration by using the Fourier expansion of the factors $e^{-i k_{b} \cdot \alpha_{0} \sin (\omega t)}$, we obtain

$$
S_{i o n}^{B_{1}}=i(2 \pi)^{-1} \sum_{\ell=-\infty}^{\ell=+\infty} \delta\left(E_{k_{a}}+E_{k_{b}}-E_{k_{i}}-E_{0}-\ell \omega\right) f_{i o n}^{B_{1}, \ell}
$$


here $f_{i o n}^{B_{1}, \ell}$ is the first Born amplitude for the laser-assisted (e, 2e) scattering amplitude with the transfer of $\ell$ photons. This quantity is given by

$$
f_{i o n}^{B_{1}, \ell}=f_{1}+f_{2}+f_{3}
$$

with

$$
\begin{gathered}
f_{1}=-2 \Delta^{-2} J_{\ell}(\lambda)\left\langle\psi_{c, k_{b}}^{(-)}|\exp (i \Delta . \mathbf{r})| \psi_{0}\right\rangle, \\
f_{2}=i \Delta^{-2} \sum_{n}\left\langle\psi_{c, k_{b}}^{(-)}|\exp (i \Delta . \mathbf{r})| \psi_{n}\right\rangle M_{n 0}\left(\frac{J_{\ell-l}(\lambda)}{E_{n}-E_{0}-\omega}-\frac{J_{\ell+l}(\lambda)}{E_{n}-E_{0}+\omega}\right),
\end{gathered}
$$

and

$$
\begin{gathered}
f_{3}=i \Delta^{-2} \sum_{n}\left\langle\psi_{n}|\exp (i \Delta . \mathbf{r})| \psi_{0}\right\rangle M_{n k_{b}}^{*}\left(\frac{J_{\ell-l}(\lambda)}{E_{n}-E_{k_{b}}+\omega}-\frac{J_{\ell+l}(\lambda)}{E_{n}-E_{k_{b}}-\omega}\right) \\
-2 \Delta^{-2} \boldsymbol{k}_{b} \cdot \boldsymbol{\alpha}_{0} J_{\ell}^{\prime}(\lambda)\left\langle\psi_{c, k_{b}}^{(-)}|\exp (i \Delta . \mathbf{r})| \psi_{0}\right\rangle .
\end{gathered}
$$

In these equations, $J_{\ell}$ is a Bessel function of order $\ell, J_{\ell}^{\prime}$ is its first derivative, and we have introduced the quantity $\lambda=\left(\Delta-\boldsymbol{k}_{b}\right) \cdot \boldsymbol{\alpha}_{0}$, where $\Delta=\boldsymbol{k}_{i}-\boldsymbol{k}_{a}$ is the momentum transfer of the collision.

The case of the absorption $(\ell=1)$ or emission $(\ell=-1)$ of one laser photon by the electron-atom system is of particular interest. Indeed, in addition to the expressions (26a)-(26c) which are valid to all orders in the laser-projectile interaction and to first order in the laser-atom and electron-atom interactions, one can then also obtain the lowest-order perturbative version of these amplitudes by using a second-order treatment in the combined electron-atom and laser-atom system (electron + atom) interactions. The corresponding amplitudes are given for the case $\ell=1$ by

$$
\begin{gathered}
f_{1}=-\Delta^{-2} \omega^{-2} \Delta \cdot \mathcal{E}_{0}\left\langle\psi_{c, k_{b}}^{(-)}|\exp (i \Delta . \mathbf{r})| \psi_{0}\right\rangle, \\
f_{2}=-i \Delta^{-2}\left\langle\psi_{c, k_{b}}^{(-)}\left|\exp (i \Delta . \mathbf{r}) G_{c}\left(E_{0}+\omega\right) \mathcal{E}_{0} . \boldsymbol{r}\right| \psi_{0}\right\rangle,
\end{gathered}
$$

and

$$
f_{3}=-i \Delta^{-2}\left\langle\psi_{c, k_{b}}^{(-)}\left|\mathcal{E}_{0} \cdot \mathbf{r} G_{c}\left(E_{k_{b}}-\omega\right) \exp (i \Delta . \mathbf{r})\right| \psi_{0}\right\rangle .
$$

where $G_{c}$ denotes the Coulomb Green's function. For the case $\ell=-1$ (emission of one photon) the corresponding formulas are obtained by changing $\omega$ into $-\omega$. We remark that these limiting forms can be recovered directly from the corresponding amplitudes of Equation (26a)-(26c) by retaining only terms up to first order (in the field strength) in the power series expansions of the Bessel functions.

The first Born triple differential cross section corresponding to the $(\mathrm{e}, 2 \mathrm{e})$ reaction accompanied by the transfer of $\ell$ photons is then given by

$$
\frac{d^{3} \sigma_{i o n}^{B_{1}, \ell}}{d \Omega_{a} d \Omega_{b} d E}=\frac{k_{a} k_{b}}{k_{i}}\left|f_{i o n}^{B_{1}, \ell}\right|^{2}
$$

We remark that the amplitude $f_{1}$ corresponds to a first Born treatment in which the target dressing effects are neglected. In this approximation, the first Born TDCS reduces to

$$
\begin{aligned}
\left(\frac{d^{3} \sigma^{B_{1}, \ell}}{d \Omega_{a} d \Omega_{b} d E}\right)_{\text {no dressing }} & =\frac{k_{a} k_{b}}{k_{i}}\left|f_{1}\right|^{2} . \\
& =\frac{k_{a} k_{b}}{k_{i}} J_{\ell}^{2}(\lambda)\left|f_{\text {ion }}^{B_{1}}\right|^{2} .
\end{aligned}
$$

with

$$
f_{i o n}^{B_{1}}=-\frac{2}{\Delta^{2}}\left\langle\psi_{c, k_{b}}^{(-)}|\exp (i \Delta . r)| \psi_{0}\right\rangle .
$$


is the field-free first Born ionization amplitude.

A similar analysis of the laser-assisted electron-impact ionization can be made for the second-order term of the Born series. Thus, the second Born S-matrix element accompanied by the transfer of $\ell$ photons can be given by

$$
\begin{aligned}
S_{i o n}^{B_{2}}= & -i \int_{-\infty}^{+\infty} d t \int_{-\infty}^{+\infty} d t^{\prime}\left\langle\chi_{k_{a}}\left(\boldsymbol{r}_{0}, t\right) \phi_{k_{b}}(\boldsymbol{r}, t)\right| V_{d}\left(\boldsymbol{r}_{0}, \boldsymbol{r}\right) \\
& \times G_{0}^{(+)}\left(\boldsymbol{r}_{0}, \boldsymbol{r}, t, \boldsymbol{r}_{0}^{\prime}, \boldsymbol{r}^{\prime}, t^{\prime}\right) V_{d}\left(\boldsymbol{r}_{0}^{\prime}, \boldsymbol{r}^{\prime}\right)\left|\chi_{k_{i}}\left(\boldsymbol{r}_{0}^{\prime}, t^{\prime}\right) \phi_{0}\left(\boldsymbol{r}^{\prime}, t^{\prime}\right)\right\rangle,
\end{aligned}
$$

where $G_{0}^{(+)}$is the causal propagator defined by

$$
G_{0}^{(+)}\left(r_{0}, r, t, r_{0}^{\prime}, r^{\prime}, t^{\prime}\right)=-i \Theta\left(t-t^{\prime}\right) \sum_{n} \int d \boldsymbol{q} \chi_{q}\left(r_{0}, t\right) \chi_{q}\left(r_{0}^{\prime}, t^{\prime}\right) \phi_{n}(r, t) \phi_{n}\left(r^{\prime}, t^{\prime}\right),
$$

It should be noted that this term is second order in the electron-atom interaction potential $V_{d}$, and contains atomic wave functions corrected to first-order correction in $\mathcal{E}_{0}$ for the target dressed states. If one retains a global first-order correction in $\mathcal{E}_{0}$ for the target states, one finds that $S_{i o n}^{B_{2}}$ is the sum of two terms which are respectively of zeroth and first-order in $\mathcal{E}_{0}$. We shall neglect the second-order contribution to the S-matrix element for laser-assisted collisions calculated in first order in $\mathcal{E}_{0}$, and concentrate our discussion on the computation of the dominant term $S_{i o n}^{B_{2}, 0}$, which describes the collision of a Volkov electron with the undressed atom.

Making explicit the time dependence of the matrix elements and using the integral representation of $\Theta$

$$
\Theta(t) e^{-i z t}=\frac{1}{2 \pi i} \lim _{\varepsilon \rightarrow 0^{+}} \int_{-\infty}^{+\infty} d \xi \frac{\exp (-i \xi t)}{z-\xi-i \varepsilon}
$$

one obtains

$$
\begin{aligned}
S_{i o n}^{B_{2}, 0} & =\frac{1}{(2 \pi)^{4}} \sum_{n} \int d \boldsymbol{q} \int_{-\infty}^{+\infty} d t \int_{-\infty}^{+\infty} d t^{\prime} \\
& \times \frac{1}{2 \pi i} \int_{-\infty}^{+\infty} d \xi \frac{\exp \left(-i \xi\left(t-t^{\prime}\right)\right)}{E_{q}+E_{n}-\xi-i \varepsilon} \exp \left(i \boldsymbol{\alpha}_{0} \cdot \boldsymbol{p}_{b} \sin (\omega t)\right) \\
& \times \exp \left[i\left\{\left(E_{k_{a}}+E_{k_{b}}\right) t-\boldsymbol{\alpha}_{0} \cdot \Delta_{f} \sin (\omega t)\right\}\right] \\
& \times \exp \left[-i\left\{\left(E_{k_{i}}+E_{0}\right) t^{\prime}+\boldsymbol{\alpha}_{0} \cdot \Delta_{i} \sin \left(\omega t^{\prime}\right)\right\}\right] \\
& \times \frac{\left\langle\psi_{c, p_{b}}^{(-)}\left|\widetilde{V}_{d}\left(\Delta_{f}, \boldsymbol{r}\right)\right| \psi_{n}\right\rangle\left\langle\psi_{n}\left|\widetilde{V}_{d}\left(\Delta_{i}, \boldsymbol{r}\right)\right| \psi_{0}\right\rangle}{\Delta_{f}^{2} \Delta_{i}^{2}}
\end{aligned}
$$

with $\Delta_{i}=\boldsymbol{k}_{i}-\boldsymbol{q}, \Delta_{f}=\boldsymbol{q}-\boldsymbol{k}_{a}$, and $\widetilde{V}_{d}(\Delta, \boldsymbol{r})=e^{i \Delta \mathrm{r}}-1$. The expression (31) is obtained from the quantity Equation (34) by shifting the pole of the integrand, respectively, below and above the real $\omega$-axis by a small positive quantity $\varepsilon \rightarrow 0^{+}$. The sum over $n$ runs over the complete set of hydrogenic states, and one has to integrate over the virtual projectile states $\mid \chi_{q}\left(\mathbf{r}_{0}, t\right)>$ with wave vectors $\boldsymbol{q}$. After using the generating function expansion

$$
\exp [i z \sin (\omega t)]=\sum_{n=-\infty}^{+\infty} J_{n}(z) \exp (i n \omega t),
$$


for each of the sinusoidal exponentials, the $t^{\prime}$ integral reduces to a $\delta$ function. This makes it trivial to perform the $\xi$ integration for $\xi=E_{k_{i}}+E_{0}-E_{k_{b}}+\ell^{\prime \prime} \omega$ with considering the exchange of $\ell=\ell^{\prime}+\ell^{\prime \prime}$ photons, the corresponding second Born S-matrix component then reads

$$
\begin{aligned}
S_{i o n}^{B_{2}, 0} & =\frac{i}{(2 \pi)^{3}} \sum_{\ell=-\infty}^{\ell=+\infty} \delta\left(E_{k_{a}}+E_{k_{b}}-E_{k_{i}}-E_{0}-\ell \omega\right) \\
& \times \sum_{\ell^{\prime}=-\infty}^{\ell^{\prime}=+\infty} \int d \boldsymbol{q} \frac{J_{\ell-\ell^{\prime}}\left(\boldsymbol{\alpha}_{0} . \Delta_{i}\right) J_{\ell^{\prime}}\left(\boldsymbol{\alpha}_{0} \cdot\left(\Delta_{f}-\boldsymbol{k}_{b}\right)\right)}{\Delta_{i}^{2} \Delta_{f}^{2}} \\
& \times \sum_{n} \frac{\left\langle\psi_{c, p_{b}}^{(-)}\left|\widetilde{V}_{d}\left(\Delta_{f}, \boldsymbol{r}\right)\right| \psi_{n}\right\rangle\left\langle\psi_{n}\left|\widetilde{V}_{d}\left(\Delta_{i}, \boldsymbol{r}\right)\right| \psi_{0}\right\rangle}{E_{q}+E_{k_{b}}-E_{k_{i}}+\omega_{n 0}-\left(\ell-\ell^{\prime}\right) \omega-i \varepsilon} .
\end{aligned}
$$

Using the soft-photon approximation [84] which assumes that the quantity $\ell^{\prime} \omega$ entering the denominator of the second-order matrix element in Equation (36) is much smaller than any energy difference $\ell^{\prime} \omega \ll\left|E_{k_{i}}+E_{0}-E_{q}-E_{k_{b}}+\ell \omega-E_{n}\right|$, and performing the infinite summation over $\ell^{\prime}$ by using the addition theorem for Bessel function

$$
J_{n}(u+v)=\sum_{m} J_{m}(u) J_{n-m}(v),
$$

Thus, the lowest-order component $S_{i o n}^{B_{2}, 0}$ evaluated at the shifted momenta $\Delta_{i}$ and $\Delta_{f}$ can be expressed in terms of a second-Born amplitude as

$$
S_{i o n}^{B_{2}, 0}=-(2 \pi)^{-1} i \sum_{\ell=-\infty}^{\ell=+\infty} \delta\left(E_{k_{a}}-E_{k_{b}}-E_{k_{i}}-E_{0}-\ell \omega\right) f_{i o n}^{B_{2}, \ell, 0}(\Delta),
$$

where

$$
f_{\text {ion }}^{B_{2}, \ell, 0}(\Delta)=J_{\ell}(\lambda) f_{\text {ion }}^{B_{2}, 0}(\Delta)
$$

with

$$
f_{i o n}^{B_{2}, 0}(\Delta)=-\frac{1}{\pi^{2}} \int_{0}^{+\infty} q^{2} d q d \tilde{\zeta}_{q}^{\prime} \frac{\left\langle\psi_{c, p_{b}}^{(-)}\left|\widetilde{V}_{d}\left(\Delta_{f}, \boldsymbol{r}\right) G_{c}(\Omega) \widetilde{V}_{d}\left(\Delta_{i}, \boldsymbol{r}\right)\right| \psi_{0}\right\rangle}{\Delta_{i}^{2} \Delta_{f}^{2}} .
$$

is the field-free second-Born ionization amplitude evaluated at the shifted momenta $\Delta_{i}$ and $\Delta_{f}$.

$$
G_{c}(\Omega)=\sum_{n} \frac{\left|\psi_{n}\right\rangle\left\langle\psi_{n}\right|}{\Omega-E_{n}}
$$

is the Coulomb Green's function with argument $\Omega=E_{k_{i}}-E_{k_{b}}-E_{q}+E_{0}+\ell \omega$, where $E_{0}=-0.5$ a.u. is the ground state energy of atomic hydrogen, and $E_{q}$ is the virtual projectile energy. We have the definition $\lambda=\boldsymbol{\alpha}_{0} .\left(\Delta-\boldsymbol{k}_{b}\right)$ with $\Delta=\Delta_{i}+\Delta_{f}$.

The electron-atom amplitude with the transfer of $\ell$ photons may be written in the second Born approximation as

$$
f_{\text {ion }}^{\ell}(\Delta)=f_{\text {ion }}^{B_{1}, \ell}(\Delta)+f_{\text {ion }}^{B_{2}, \ell, 0}(\Delta)
$$

We note that the integral in Equation (40) over the virtual projectile states $\chi_{q}\left(\mathbf{r}_{0}, t\right)$ with wave vector $\boldsymbol{q}$ is prohibitively difficult, which is actually zero at some values of incident electron energies. We shall overcome this difficult by using the exact upper boundary of the integral Equation (40) over the virtual projectile energies (see Appendix B), which is obtained by the requirement

$$
E_{q} \leq \inf \left(E_{k_{i}}, E_{k_{a}}\right) .
$$


The first and second Born amplitudes corresponding to the first and second-order contributions to the S-matrix element, for the laser-assisted electron-impact ionization, have been computed exactly without further approximation with the help of a Sturmian approach described in Appendix A.

The contribution of laser-assisted (e, 2e) collisions to the S-matrix of exchange scattering leads to some conceptual difficulties but would not significantly alter the results of the present discussion. We have considered in the present work a first Born exchange amplitude $g_{i o n}^{\ell}$ with the transfer of $\ell$ photons [79,85]

$$
g_{\text {ion }}^{\ell}(\Delta) \simeq J_{\ell}(\lambda) g_{\text {ion }}^{\text {Och }},
$$

where

$$
g_{\text {ion }}^{\text {Och }}=\frac{\Delta^{2}}{k_{i}^{2}} f_{i o n}^{B_{1}} .
$$

Finally, the second Born triple differential cross section corresponding to the ionization process, with the transfer of $\ell$ photons, is given by

$$
\frac{d^{3} \sigma_{i o n}^{B_{2}, \ell}}{d \Omega_{a} d \Omega_{b} d E}=\frac{k_{a} k_{b}}{k_{i}}\left(\frac{1}{4}\left|f_{\text {ion }}^{\ell}+g_{\text {ion }}^{\ell}\right|^{2}+\frac{3}{4}\left|f_{\text {ion }}^{\ell}-g_{\text {ion }}^{\ell}\right|^{2}\right)
$$

\subsection{Results and Discussion}

Let us turn to a discussion of the results obtained in the case of $(\mathrm{e}, 2 \mathrm{e})$ collisions in several geometrical configurations. In the present investigation, our results are interpreted by estimating the first and second Born triple differential cross sections, where the scattering angle is kept fixed at $5^{\circ}$. We present and analyze our findings for the TDCS of the laser-assisted $(\mathrm{e}, 2 \mathrm{e})$ reaction in the coplanar asymmetric geometry. Without loss of generality, we assume the origin of the coordinate system to be the target nucleus and the $z$-axis to be along the incident momentum. The $x$-axis is in the plane defined by the incident momentum and the polarization vector of the external field. The scattering angle of the scattered electron and the emission angle of the ejected electron are denoted respectively by $\theta_{a}$ and $\theta_{b}$. The former is measured in anticlockwise direction, and the latter clockwise.

In Figure 3, we give the triple differential cross sections corresponding to the ionization of the atomic hydrogen from the ground state by electron-impact, in the presence of a laser field, as a function of the ejected electron angle $\theta_{b}$. The incident electron energy is $E_{k_{i}}=40 \mathrm{eV}$, the ejected electron energy is $E_{k_{b}}=5 \mathrm{eV}$, the scattering angle is $\theta_{a}=5^{\circ}$, and the laser photon energy is $\omega=1.17 \mathrm{eV}$ which corresponds to first harmonic of Nd-YAG laser. We are working in a geometry in which the polarization vector $\hat{\varepsilon}$ of the field (which is along $\mathcal{E}_{0}$ for the case of linear polarization considered here) is parallel to the incident momentum $\mathbf{k}_{i}$. We present the results of our complete computation of the TDCS in the second Born approximation (SBA), and compared them with the first Born approximation (FBA) ones, and with those obtained when ignoring the dressing of the target. One remarks that the values obtained from the simplified treatment ignoring the dressing of the target correctly reproduce the shape of the angular distribution although the magnitudes of the cross sections are underestimated. With no net exchange of photons, the results show a distinct two peaks structure. The binary peak is attributed to the electron-electron interaction, while the recoil peak is governed by the attraction between the electron and the nucleus. We also remark that when the laser field is applied, but with no photon exchange, the results are governed by binary collisions. The comparison between FBA and SBA results indicates that the binary peak is strongly enhanced in SBA while the recoil peak is suppressed slightly. With the net exchange of one photon $(\ell= \pm 1)$, the recoil collision becomes important and the dressing of the atomic target can also significantly affect the TDCS corresponding to the ionizing process. This is shown in Figure 3, where both the shape and the magnitude of our FBA and SBA cross sections exhibit important departures with respect to the results obtained by ignoring dressing effects for a laser frequency $\omega=1.17 \mathrm{eV}$ and an electric field strength $\mathcal{E}_{0}=10^{7} \mathrm{~V} / \mathrm{cm}$. 

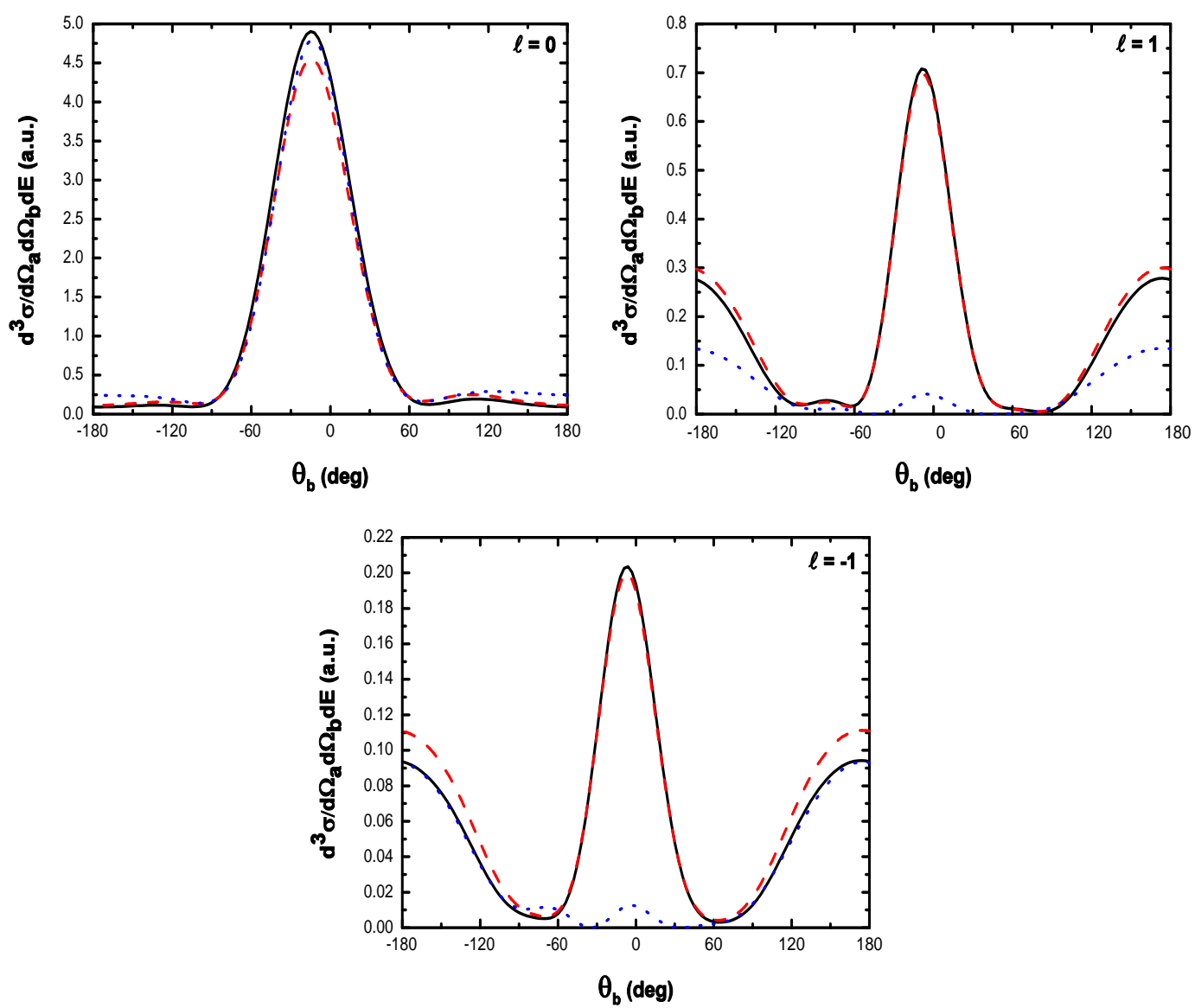

Figure 3. Triple differential cross sections corresponding to the laser-assisted electron-impact ionization process of atomic hydrogen as a function of the ejected angle $\theta_{b}$. The incident electron energy is $E_{k_{i}}=40 \mathrm{eV}$, the ejected electron energy is $E_{k_{b}}=5 \mathrm{eV}$, and the scattering angle is $\theta_{a}=5^{\circ}$. The laser frequency is $\omega=1.17 \mathrm{eV}$ and the electric field strength is est $\mathcal{E}_{0}=1 \times 10^{7} \mathrm{~V} / \mathrm{cm}$. The laser polarization vector of the field is set parallel to the wave vector of the incident electron $\left(\hat{\varepsilon} / / k_{i}\right)$. —: second Born approximation results. --- : first Born approximation results. $\cdots \cdots$ : results obtained by ignoring the dressing of the target.

In Figures 4-6, we give the triple differential cross sections as a function of the ejected angle $\theta_{b}$ and for different incident energies $E_{i}=30,50$ and $100 \mathrm{eV}$ with and without exchange of photons $(\ell=0,1,-1)$. One remarks that, with the increase of $|\ell|$, the recoil collision becomes prominent. As is already noted before by several authors $[29,32,76]$, the dressing effects are seen to be important with the exchange of one photon $(\ell= \pm 1)$. This is due to the presence in the atomic term in first Born approximation of $s-p$ transition amplitudes which behave like $\Delta^{-1}$ for small transfer momentum $\Delta$. We notice that, in Figure 4, when the incident electron energy decreases, the recoil peaks in the first and second Born approximations are depressed, and the difference between the values of the second Born and first Born TDCS becomes larger. In fact, the second order correction becomes significantly important in the vicinity of the maximum of the binary peaks and almost invisible elsewhere. Furthermore, the absolute magnitude of the first and second Born triple differential cross sections increases with the incident energy.

In Figures 5 and 6, the dependance of the cross sections on the ejected angle is shown for $\ell= \pm 1$. For the absorption of one photon, the angular distribution is strongly modified as the binary and recoil peaks are now split into big and smaller lobes with different magnitudes. The overall magnitude of the cross section increases steadily with the incident electron energy. Another interesting point is the fact that the binary peaks remains unchanged for the first and second Born approximation, while the 
recoil peaks marks a small change in the order of their cross sections. This indicates that, for the absorption of one photon $(\ell=1)$, and at relatively low incident energies, the electron-atom interaction in the second Born approximation does not play a dominant role in the physics of the process. For the emission of one photon $(\ell=-1)$ the laser-assisted triple differential cross sections corresponding to the $(\mathrm{e}, 2 \mathrm{e})$ reaction in atomic hydrogen show different behavior for the cross sections to the case of absorption. In fact, the magnitude of the cross sections is significantly smaller, meaning that the system absorbs net energy from the radiation background. Furthermore, a major suppression of the magnitude of the recoil peaks in the SBA with respect to that predicted by the first Born approximation is observed. Note that our second-order Born results are in excellent agreement with those obtained in the first-order Born approximation at high impact energies. We also observe the occurrence of a shift in the position of the peaks. In fact, when decreasing the incident electron energy, the maximum of the binary and recoil peaks moves to $\theta_{b}=0$ and $\theta_{b}=180-\theta_{b}$ respectively. This is due to the momentum transfer $\Delta$ who changes with the incoming electron energy (according to the energy conservation equation) leading to the observed shift of the pics to the right or left to the momentum transfer.
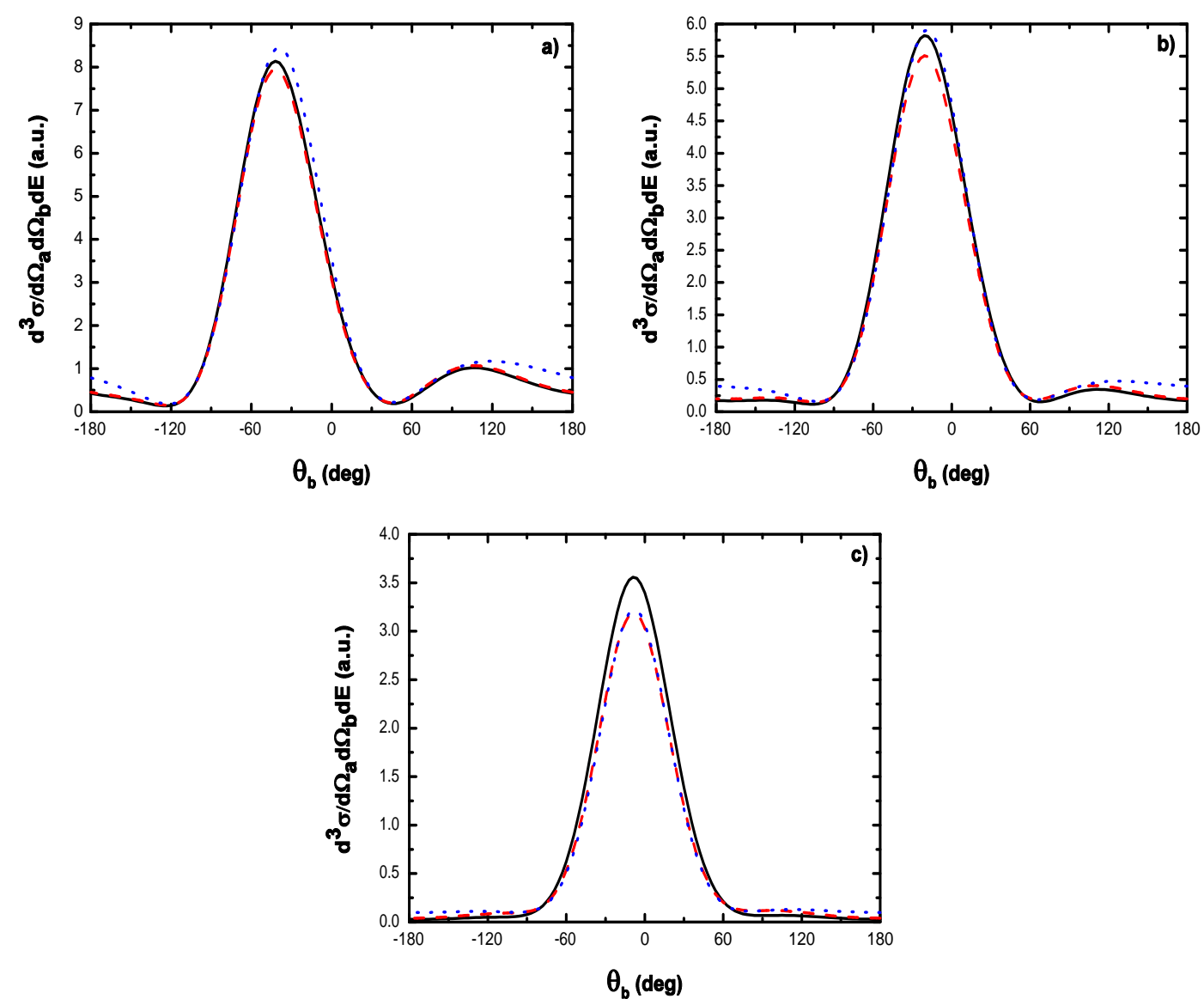

Figure 4. Triple differential cross sections corresponding to the laser-assisted electron-impact ionization process of atomic hydrogen as a function of the ejected angle $\theta_{b}$, for no net exchange of photons $(\ell=0)$ at different impact energies. The incident electron energy is (a) $E_{k_{i}}=100 \mathrm{eV},(\mathbf{b}) E_{k_{i}}=50 \mathrm{eV}$, et (c) $E_{k_{i}}=30 \mathrm{eV}$. The ejected electron energy is est $E_{k_{b}}=5 \mathrm{eV}$, and the scattering angle is $\theta_{a}=5^{\circ}$. The laser frequency is $\omega=1.17 \mathrm{eV}$ and the electric field strength is est $\mathcal{E}_{0}=1 \times 10^{7} \mathrm{~V} / \mathrm{cm}$. The laser polarization vector of the field is set parallel to the wave vector of the incident electron $\left(\hat{\varepsilon} / / k_{i}\right)$. — second Born approximation results. --- : first Born approximation results. $\cdots \cdots$ : results obtained by ignoring the dressing of the target. 

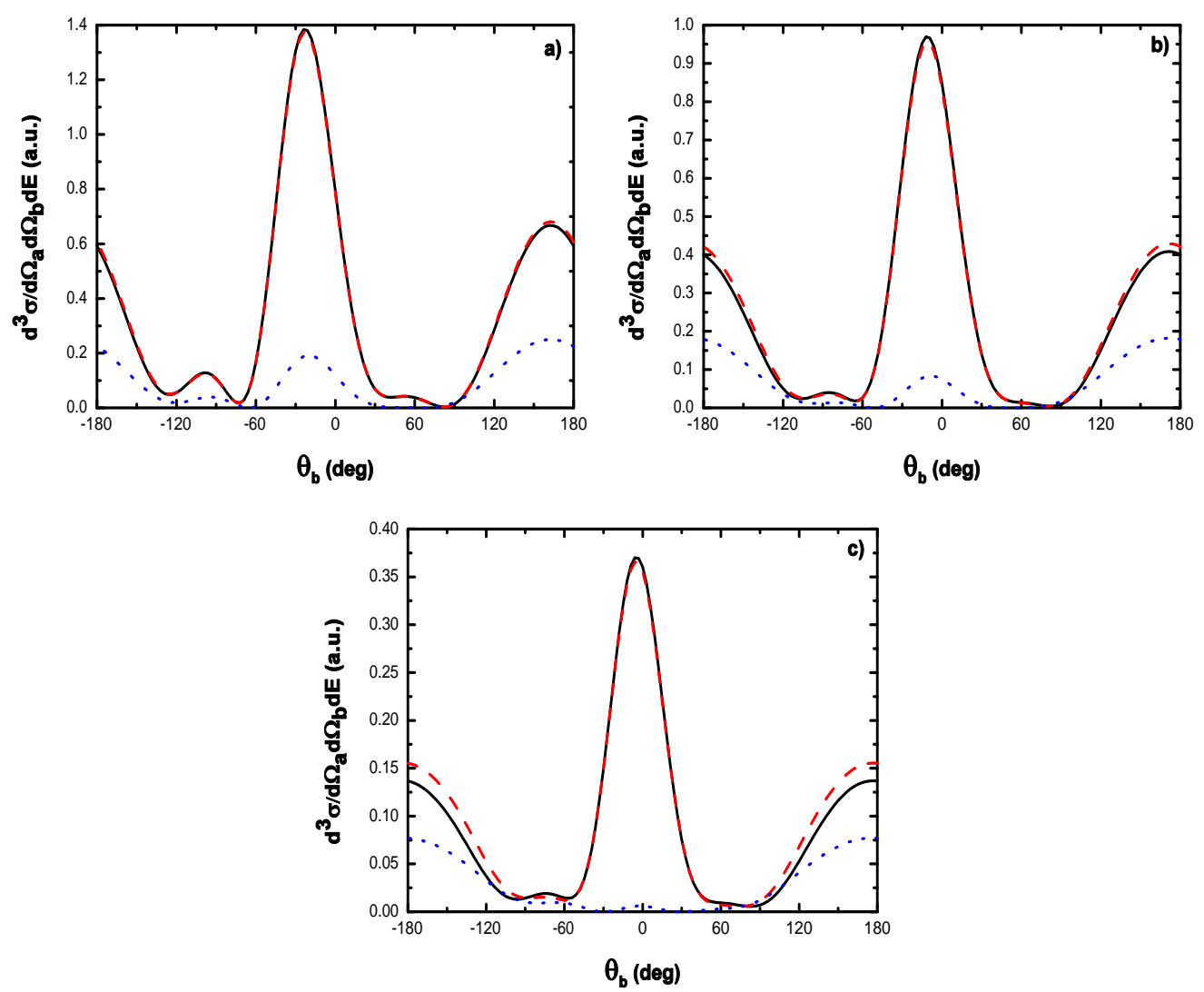

Figure 5. The same as in Figure 4, but with the absorption of one photon $(\ell=1)$.
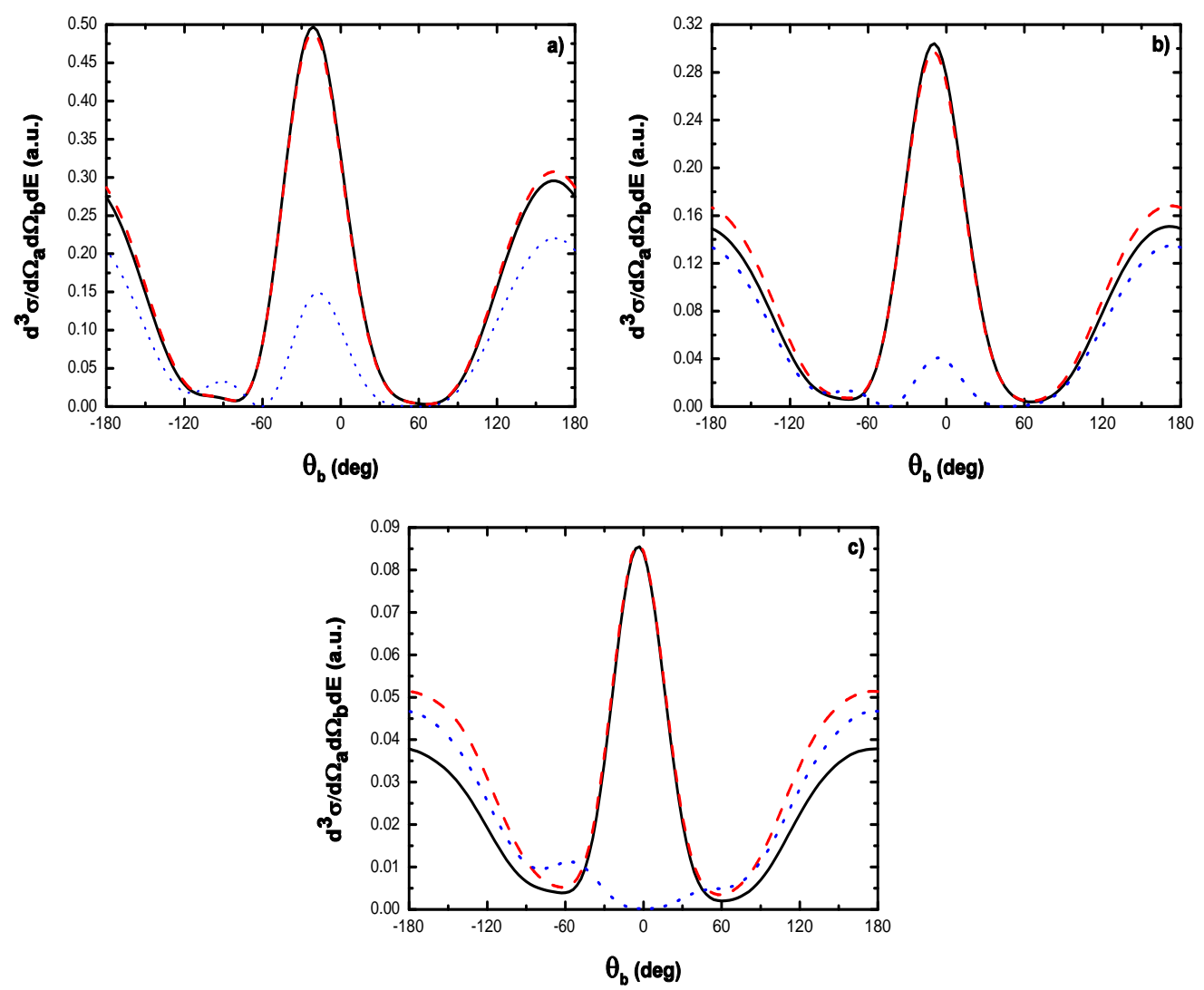

Figure 6. The same as in Figure 4, but with the emission of one photon $(\ell=-1)$. 
The last set of figures address the role of the laser polarization orientation. In Figures 7-10, we vary the polarization orientation and everything else being kept fixed. It can be observed that the geometry in which $\hat{\varepsilon}$ is kept parallel to the momentum transfer of the projectile $\Delta$ maximizes the cross section (see Figures 7 and 9). This can be understood as resulting from the fact that this geometry maximizes the argument of the Bessel functions entering the expression of the transition amplitudes. In a more physical perspective, it corresponds to the fact that the coupling between the projectile and the field is maximum for laser-assisted TDCS which are located at angles for which $\mathbf{k}_{b}$ is parallel to $\Delta$, as the coupling between the ejected electron and the field is also maximized. We also note that, in this case, the symmetry of the field-free TDCS with respect to $\Delta$ is restored. On the other hand, in the presence of the laser, the symmetry with respect to $\Delta$ is broken and one recovers the splitting of the recoil and binary peaks. The magnitudes of the cross sections, though close from each other, being notably smaller than in the preceding case. The similarity with the angular distributions in Figures 5 and 7 and in Figures 6 and 9 comes form the fact that, for the kinematics of the collision chosen here, the incident momentum $\mathbf{k}_{i}$ is almost perpendicular to $\Delta$ for every value $\mathbf{k}_{b}$. We note also that, at the laser intensity considered here, dressing effects are seen once again to produce a dramatic enhancement of the cross sections, and that the approximate treatment neglecting the dressing of the target is not adequate for the geometries considered here.
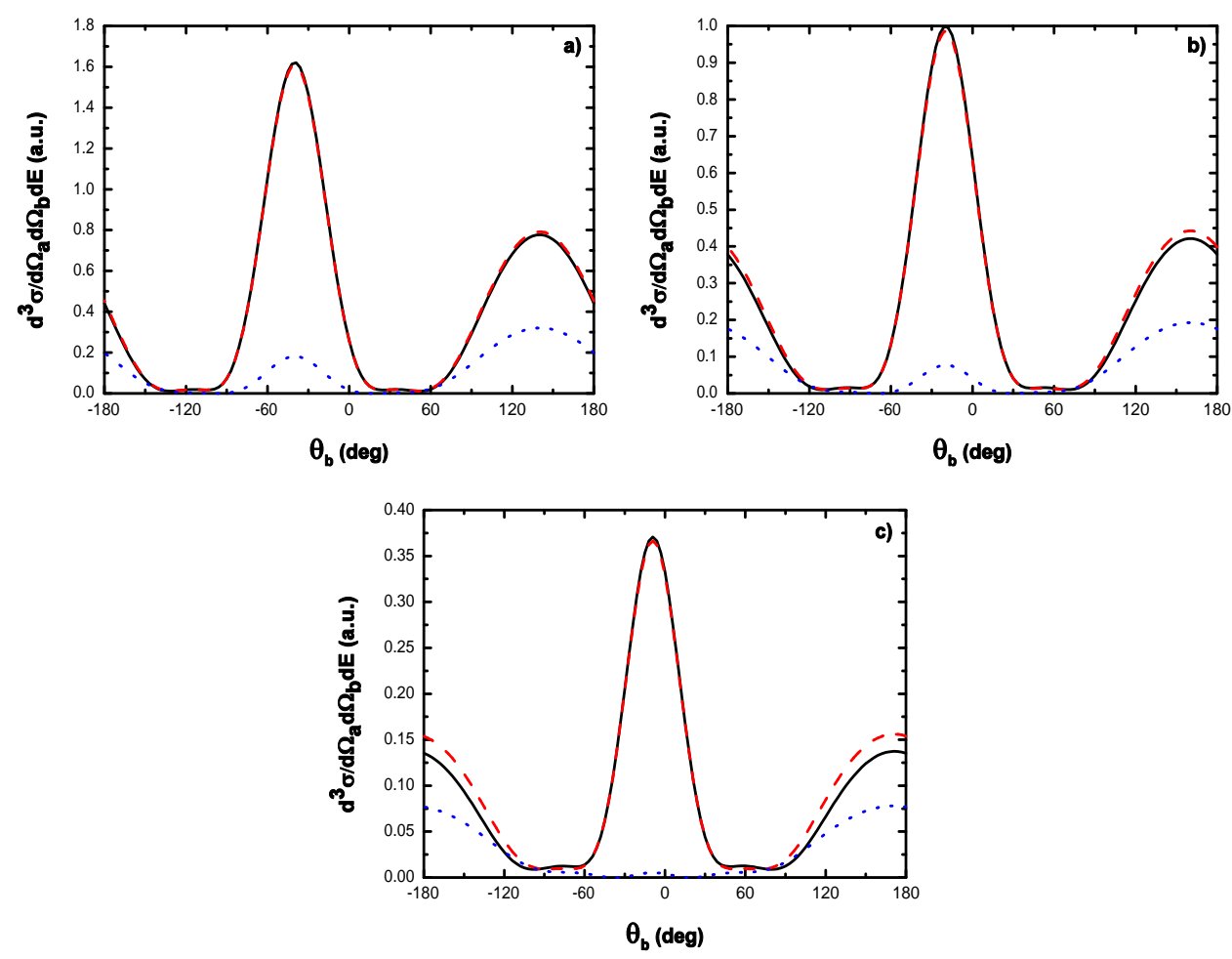

Figure 7. The same as in Figure 5, but the laser polarization vector is parallel to the momentum transfer $(\hat{\varepsilon} / / \Delta)$.

The Bessel function occurring in different amplitudes causes the oscillating structure in the results for a fixed laser field strength. When the magnitude of the total momentum transfer $\Delta$ (the recoil ion momentum) is large, the Bessel functions arguments varies in a wide range when the electron angle is scanned, leading thus to the observed oscillations in TDCS. The curves for $\ell$ and $-\ell$ present similar features since $J_{-\ell}(\lambda)=(-1)^{\ell} J_{\ell}(\lambda)$. Nevertheless, the magnitudes of the cross sections for $\ell$ and $-\ell$ are different. The origin of this difference lies in the terms in different amplitudes other than $J_{\ell}(\lambda)$. For a different direction of the electric field, i.e., different direction of $\boldsymbol{\alpha}_{0}$, the scalar product $\Delta . \boldsymbol{\alpha}_{0}$ may become sizeable, and hence the difference seen between $\hat{\varepsilon} / / \Delta$ and $\hat{\varepsilon} \perp \Delta$. It is generally believed that the recoil peak involves significant interaction with the residual ion. 

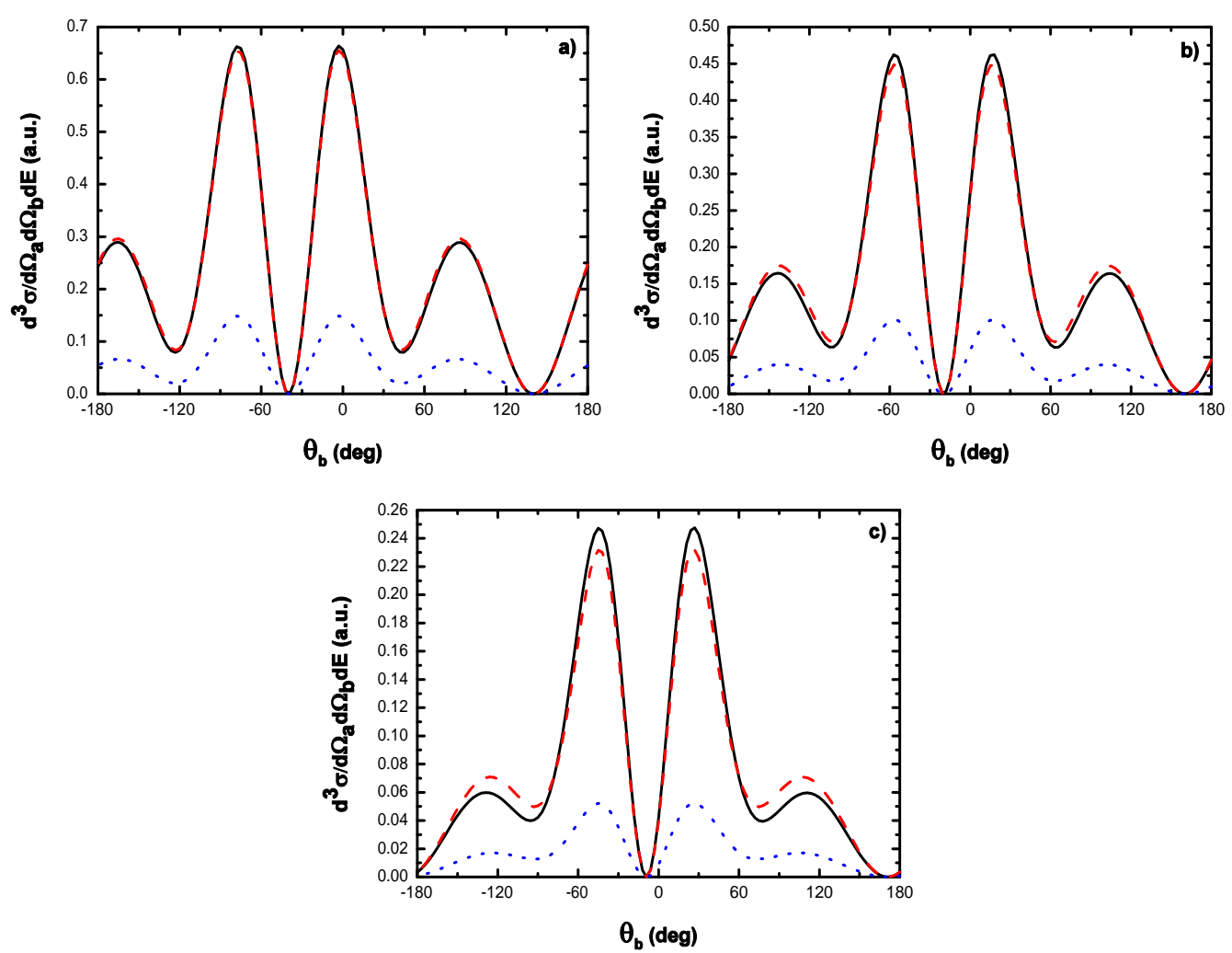

Figure 8. The same as in Figure 5, but the laser polarization vector is perpendicular to the momentum transfer $(\hat{\varepsilon} \perp \Delta)$.
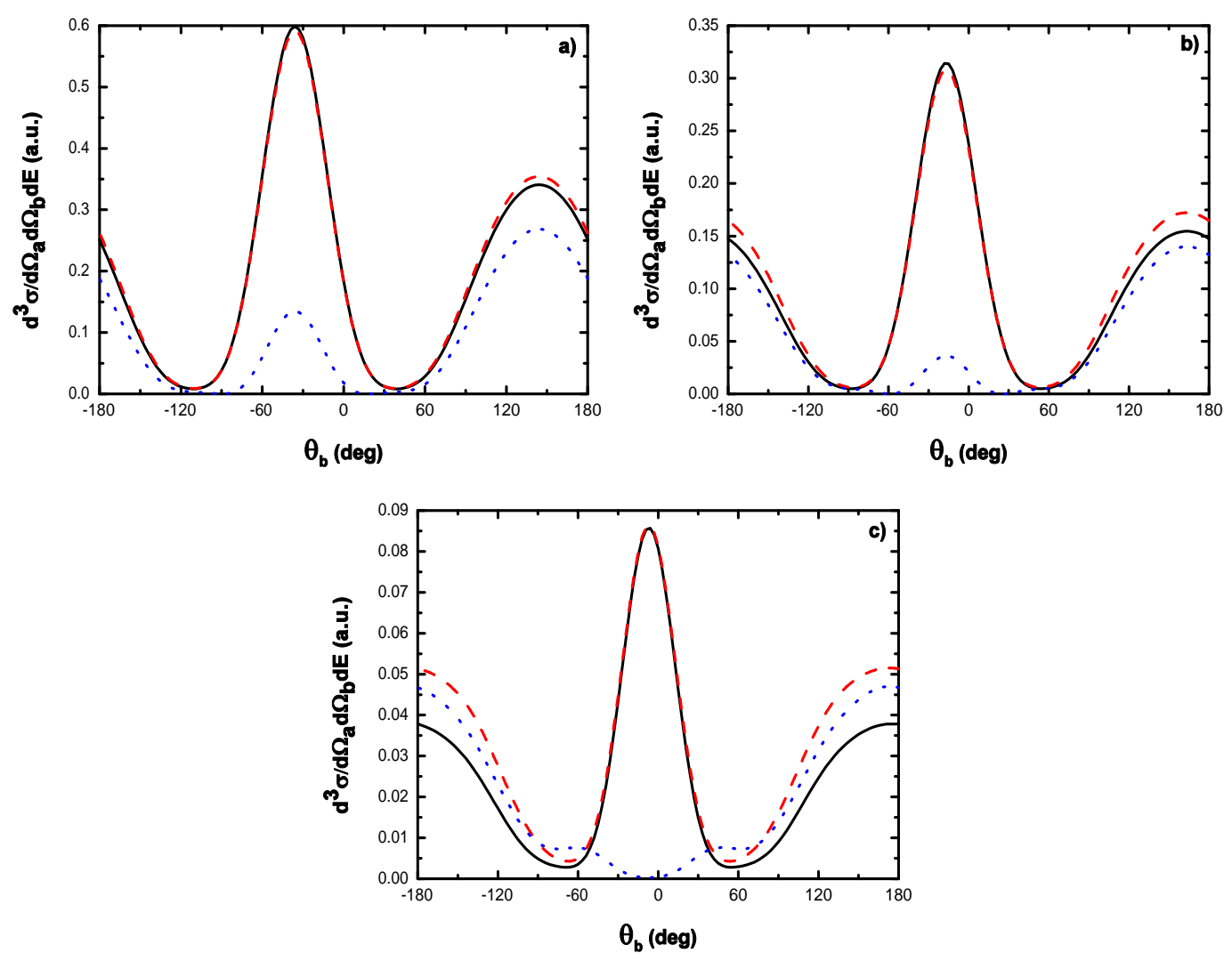

Figure 9. The same as in Figure 6, but the laser polarization vector is parallel to the momentum transfer $(\hat{\varepsilon} / / \Delta)$. 
It is seen in the set of Figures 7-10 that the target dressing has a relatively large effect on the cross sections. When the laser polarization is parallel to the momentum transfer (see Figures 7 and 9), the difference between the FBA and SBA triple differential cross sections persists in the low energies of the incident electron region in the vicinity of the recoil peaks. Note that, for the case of the absorption of one photon $(\ell=1)$ the second-order correction is small. When the polarization vector of the field is perpendicular to the momentum transfer (see Figures 8 and 10), a significant difference is noticed between the triple differential cross sections predicted by the second and first-order Born approximations at energies $\leq 50 \mathrm{eV}$ especially for $(\ell=-1)$. In fact, the margins between the SBA and FBA results are large at the maxima of the binary and recoil peaks. With impact energy decreasing, the binary collision is enhanced and the recoil collision is suppressed. To sum up, for a particular choice of the scattering geometry $(\hat{\varepsilon} \perp \Delta)$, the absolute magnitudes of the binary and recoil peaks are given extremely well by our method, while the first Born approximation is seen to be unsatisfactory. These results clearly demonstrate the importance of second-order effects in understanding the dynamics of the ionization processes at low energies. Note that the effect of the second term of the Born series decreases with the increase of the incident energy.
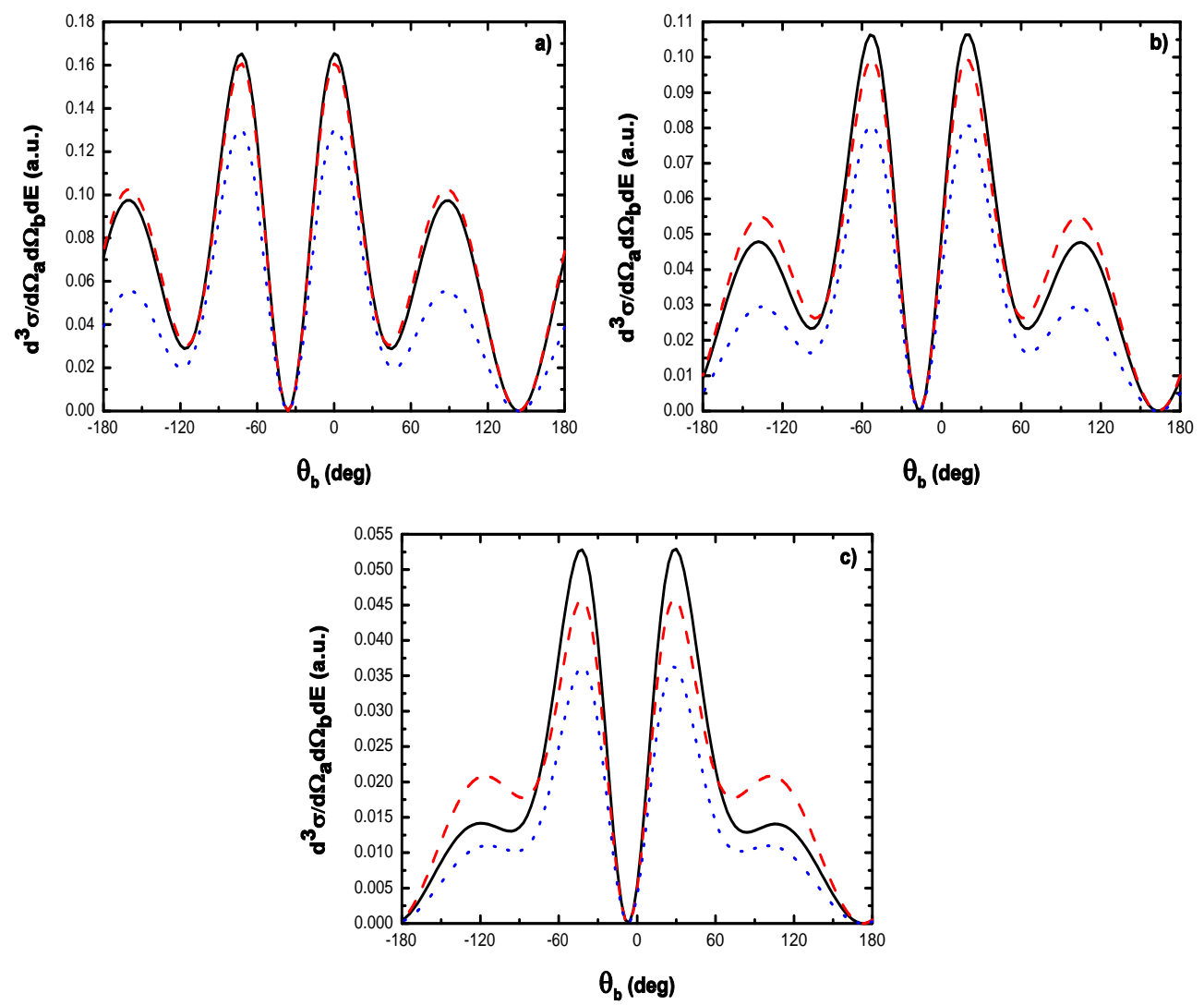

Figure 10. The same as in Figure 6, but the laser polarization vector is perpendicular to the momentum transfer $(\hat{\varepsilon} \perp \Delta)$.

In Figure 11, we give the triple differential cross sections corresponding to the laser-assisted electron-impact ionization of a hydrogen target as a function of the incident electron energies, where the angle of the ejected electron is kept fixed. The polarization vector of the field $\hat{\varepsilon}$ (which is along $\mathcal{E}_{0}$ for the case of linear polarization considered here) is set parallel to the impact momentum $\mathbf{k}_{i}$. The complete results obtained by using the ionization amplitude Equations (25) and (42) for the first and second Born approximations are compared with those obtained by neglecting the dressing of the target which coincide with the electronic amplitude $f_{1}$ and with field-free results. It is interesting to note that the results are notably sensitive to the second Born approximation at low incident electron energies. As the incident energy increases, the ratio of the second Born triple differential cross section to the first one becomes 
smaller, and at high incoming energies such proportionality factor is completely absent, where the second Born approximation does not offer a significant improvement over the first Born treatment.
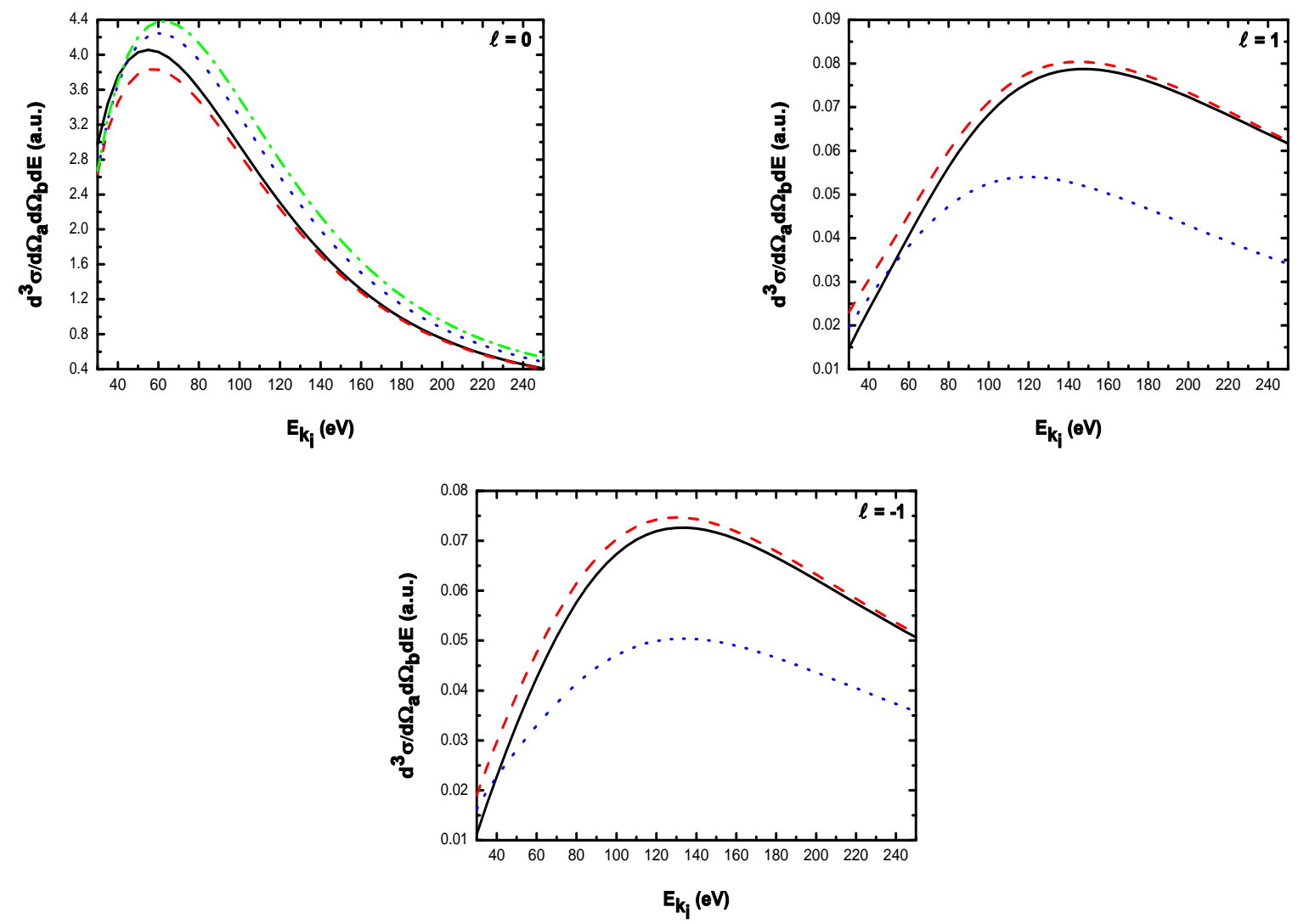

Figure 11. Triple differential cross sections corresponding to the laser-assisted electron-impact ionization process of atomic hydrogen as a function of the incident electron energy $E_{k_{i}}$. The laser frequency is $1.17 \mathrm{eV}$, the electric field strength is $\mathcal{E}_{0}=1 \times 10^{7} \mathrm{~V} / \mathrm{cm}$. The ejected electron energy is $E_{k_{b}}=5 \mathrm{eV}$, the scattering angle is $\theta_{a}=5^{\circ}$, and the emission angle of the ejected electron is $\theta_{b}=-16^{\circ}$. The laser polarization of the field is set parallel to the incident momentum $\left(\hat{\varepsilon} / / k_{i}\right)$. approximation results. --- : first Born approximation results. $\cdots \cdot$ : results obtained by neglecting the dressing of the target. $-\cdot-\cdot-$ : field-free results.

According to the domain of validity of the treatment used for taking into account the laser-atom interaction, the Nd-YAG laser frequency is taken to be $\hbar \omega=0.043$ a.u. For no net transfer of photons $(\ell=0)$, our results of TDCS are nearly of the same order of magnitude as field-free TDCS, while for $|\ell|=1$ the field-free results are much larger (not presented here in our figures). This results from the fact that the laser itself does not contribute to the ionizing process. In fact, the laser redistributes the ejected electrons in new channels associated to indices $\ell \neq 0$ in the energy conservation relation Equation (2), which are accessible in the dressed continuum of the atomic target. One observes significant departures of the results obtained by the simplified treatment neglecting dressing effects with respect to those obtained by the first and second born approximations. This difference in magnitude is traced to the role played by the explicit introduction of the atomic dressing states. This directly reflects the role of the dressing of the projectile target system by the external laser field. This is one of the interesting typical signatures of the dressing of the electron-target system in the TDCS which clearly shows the effects of the internal structure of the atomic target. Such a distorted atom also acts on the projectile by a long-range dipole potential $\left(\sim 1 / r^{2}\right)$, which requires a non-perturbative treatment of laser-atom interactions. The long-range dipole potential affects mainly the distant collisions, which contribute when the energies of the primary electron are weak. Another interesting point is the fact that the overall magnitude of the cross sections corresponding to the difference between 
SBA and FBA decreases with the increase of the incident electron energies. Moreover, our second and first Born TDCS present an absolute maximum corresponding to the zero of the momentum transfer (i.e., $1 / \Delta^{2}$ ) contained in various TDCS expressions. The same remark appears in the results obtained by neglecting the dressing of the target and laser-off.

Figures 12 and 13 shows triple differential cross sections versus the field amplitude. Apparently, the $(\mathrm{e}, 2 \mathrm{e})$ reaction process can be controlled by the field strength. Two special geometries of laser polarization are considered: $\hat{\varepsilon}_{0} / / \boldsymbol{k}_{i}$ (the laser polarization vector parallel to the incident momentum) and $\hat{\varepsilon}_{0} \perp \boldsymbol{k}_{i}$ (the laser polarization vector perpendicular to the incident momentum). For no net exchange of photons, the margins between the results of the first and second Born approximations reaches maxima at several values of field strengths in the case of $\hat{\varepsilon}_{0} / / \boldsymbol{k}_{i}$, while for $\hat{\varepsilon}_{0} \perp \boldsymbol{k}_{i}$ the margins occurs at weak field strength and at $\mathcal{E}_{0}=7 \times 10^{7} \mathrm{~V} / \mathrm{cm}$. Note that, for both geometries, dressing effects become very important with the increase of the field strength, this is because the stronger is the laser the more the atomic states are distorted. The second order correction is seen to be significant in the vicinity of the maxima of the peaks and decreases with the increase of the laser field amplitude. We also observe a small influence of the laser field at low field strength with the net exchange of photons. This is due to the chosen geometry that coincides approximatively with the region of the binary peak. This situation change in the recoil peak region when even a weak field strength leads to sizeable changes in the cross section.
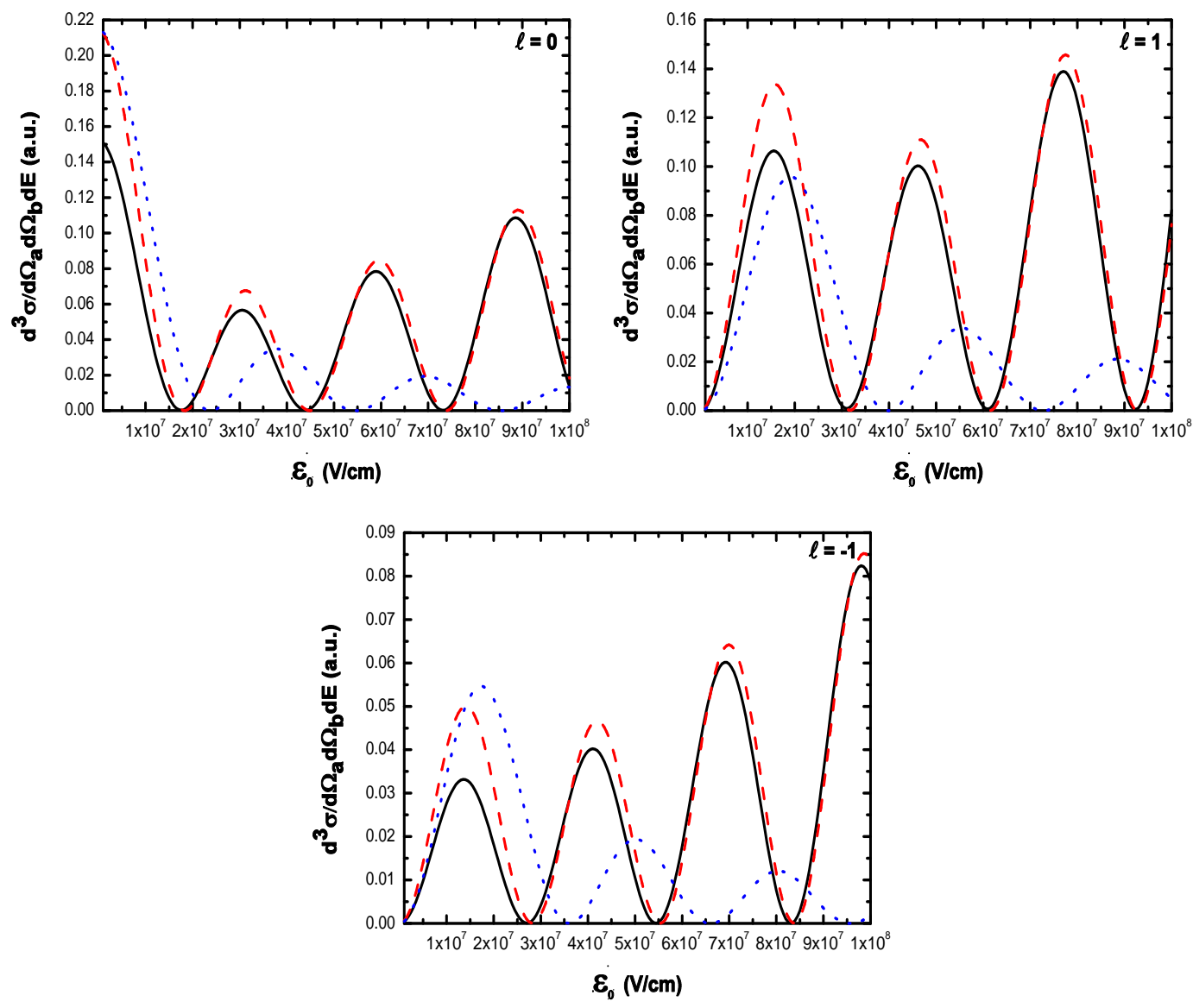

Figure 12. Triple differential cross sections corresponding to the laser-assisted electron-impact ionization process of atomic hydrogen as a function of the amplitude vector $\mathcal{E}_{0}$. the incident electron energy is $E_{k_{i}}=30 \mathrm{eV}$ and the ejected electron energy is $E_{k_{b}}=5 \mathrm{eV}$. The scattering angle is $\theta_{a}=5^{\circ}$, the emission angle of the ejected electron is $\theta_{b}=130^{\circ}$ and the laser frequency is $\omega=1.17 \mathrm{eV}$. The laser polarization of the field is set parallel to the incident momentum $\left(\hat{\varepsilon} / / \boldsymbol{k}_{\boldsymbol{i}}\right)$. — : second Born approximation results. --- : first Born approximation results. $\cdots \cdot$ : results obtained by neglecting the dressing of the target. 
In Figures 12 and 13, the corresponding dispersion curves in terms of $\mathcal{E}_{0}$ are characterized by the occurrence of sharp maxima separated by deep minima. The number of lobes increases with the laser intensity. This behavior can be traced back to the fact that the argument of the Bessel functions $J_{\ell}(\lambda)$, entering the expressions of the amplitudes Equations (25) and (42), grows with $\mathcal{E}_{0}$. By comparing Figures 12 and 13, one observes that changing the polarization orientation significantly affects the triple differential cross sections. Indeed, the amplitudes $f_{i o n}^{B_{2}, \ell, 0}$ and $f_{\text {ion }}^{B_{1}, \ell}$ depend on the laser polarization direction in a quite intricate way since $\hat{\varepsilon}_{0}$ enters their expressions though the second-order matrix elements and also via the scalar products $\boldsymbol{\alpha}_{0} . \Delta$ and $\boldsymbol{\alpha}_{0} . \boldsymbol{k}_{b}$. Dressing effects i.e., the contributions of the amplitudes $f_{2}$ and $f_{3}$ significantly affect the TDCS corresponding to the ionizing process. This observation is applied well to the SBA where the contribution of the correction term is seen to be important and improves triple differential cross sections calculations.
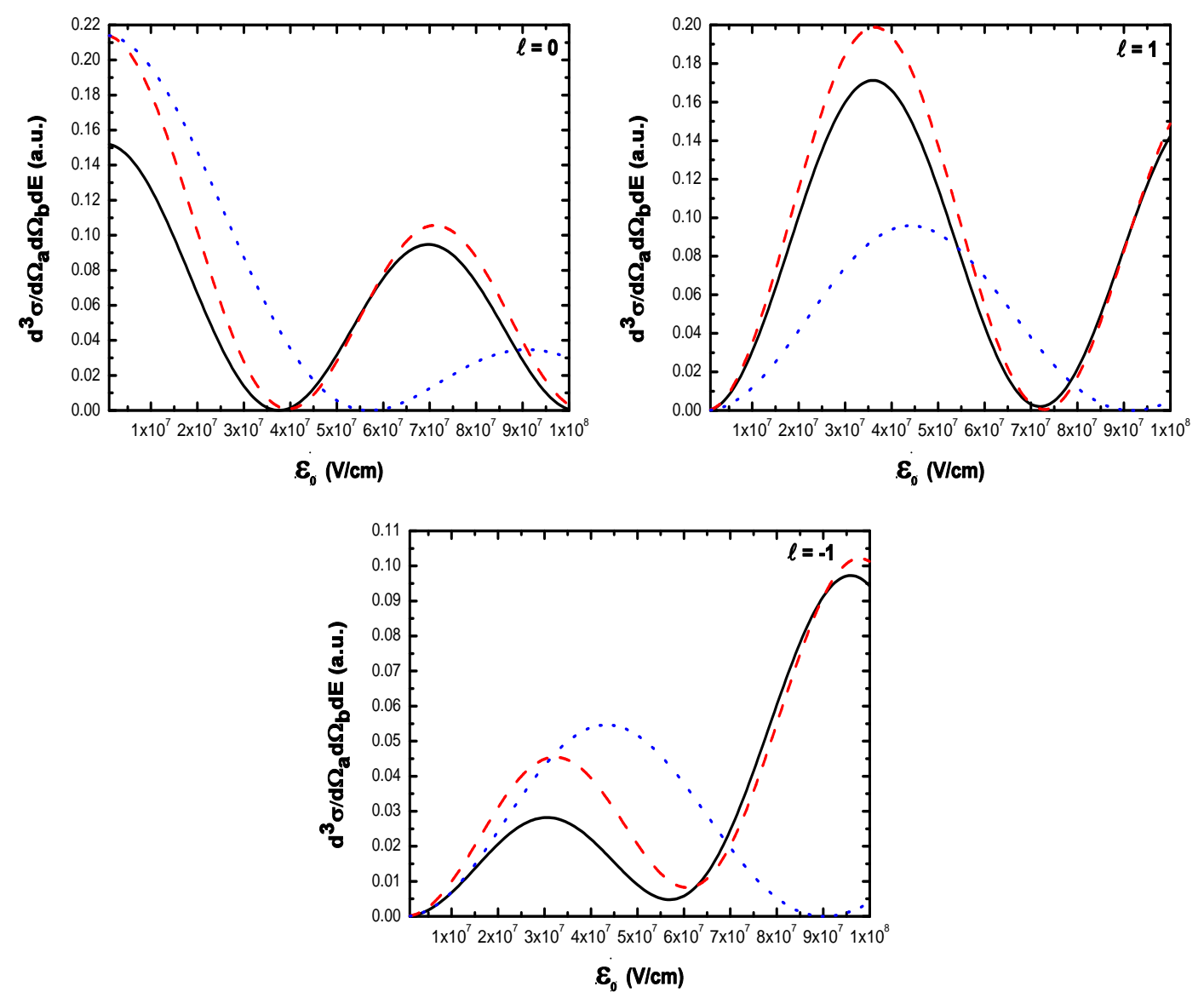

Figure 13. All the parameters are the same as Figure 12, while the laser polarization is perpendicular to the incident momentum $\left(\hat{\varepsilon} \perp k_{i}\right)$.

In Figures 14-16, we give the triple differential cross sections corresponding to the ionization of the atomic hydrogen from the ground state by electron-impact, in the presence of a laser field, as a function of the ejected electron angle $\theta_{b}$. The incident electron energy is $E_{k_{i}}=30 \mathrm{eV}$, the ejected electron energy is $E_{k_{b}}=5 \mathrm{eV}$, and the scattering angle is $\theta_{a}=5^{\circ}$. We are working in a geometry in which the polarization vector $\hat{\varepsilon}_{0}$ of the field is parallel to the incident momentum $k_{i}$. We present the results of our complete computation of the TDCS in the second Born approximation, and compare them with the first Born approximation ones, and with those obtained by neglecting the dressing effects by the laser field. One remarks that dressing effects have a relatively large effect on the cross sections with the exchange of one photon $(\ell= \pm 1)$. The frequency regime have also controlling effects on the collision process. In Figure 4, we observe that the binary peak remains unchanged for the first and second term 
of the Born series, while the recoil peaks is suppressed. Furthermore, the binary peak is dominant and the magnitude of the cross sections is considerably smaller when $(\ell=-1)$ compared to the case of the absorption of a photon $(\ell=1)$.
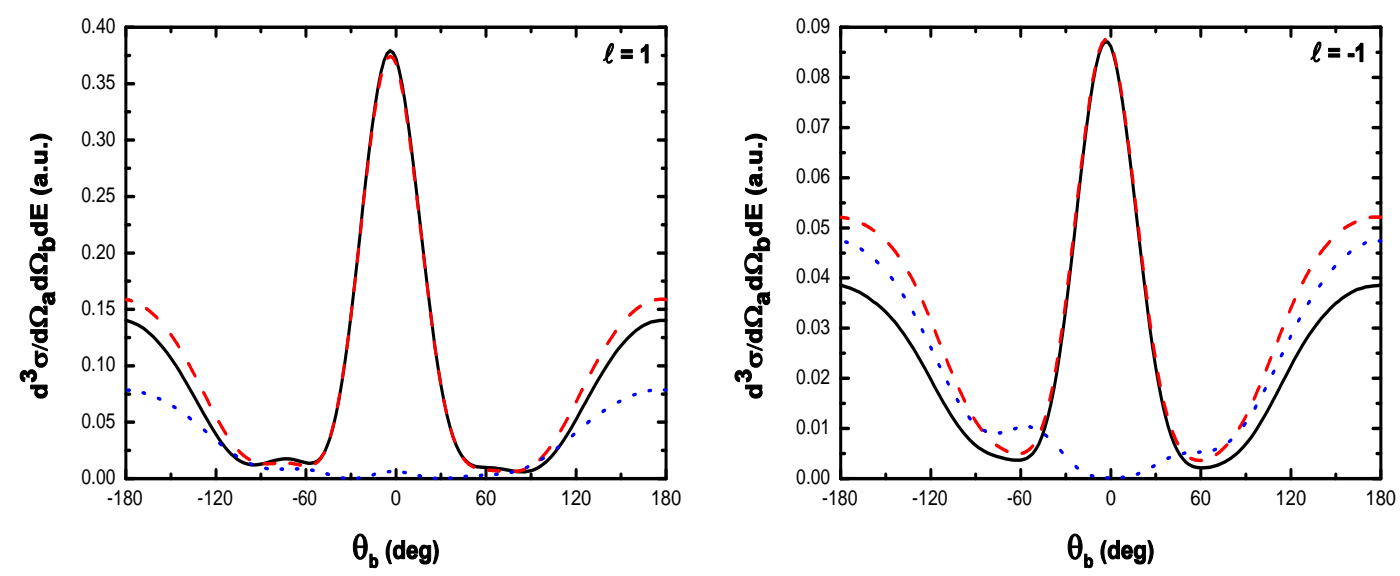

Figure 14. Triple differential cross sections corresponding to the laser-assisted electron-impact ionization process of atomic hydrogen as a function of the ejected angle $\theta_{b}$. The laser frequency $\omega=1.17 \mathrm{eV}$ and the electric field strength is $\mathcal{E}_{0}=10^{7} \mathrm{~V} / \mathrm{cm}$. The incident electron energy is $E_{k_{i}}=30 \mathrm{eV}$, the ejected electron energy is $E_{k_{b}}=5 \mathrm{eV}$, and the scattering angle is $\theta_{a}=5^{\circ}$. The laser polarization of the field is set parallel to the incident momentum $\left(\hat{\varepsilon} / / k_{i}\right) . ـ$ : second Born approximation results. --- : first Born approximation results. $\cdots \cdot$ : results obtained by neglecting the dressing of the target.
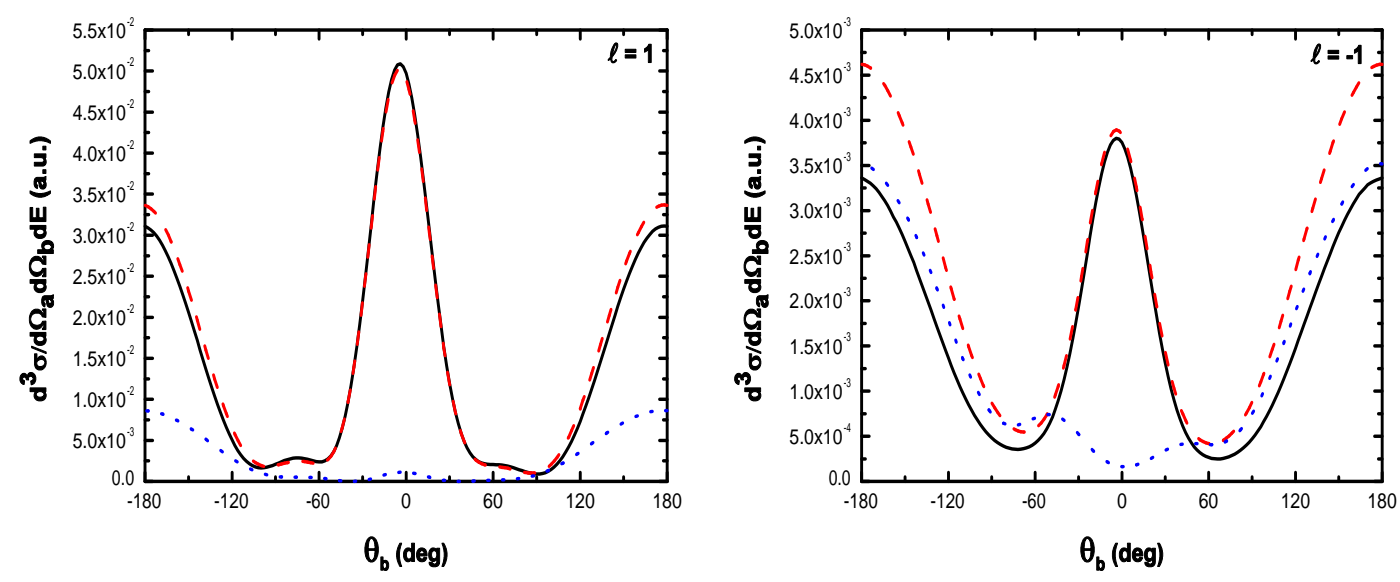

Figure 15. Same as Figure 14, but with the laser frequency $\omega=2.34 \mathrm{eV}$.
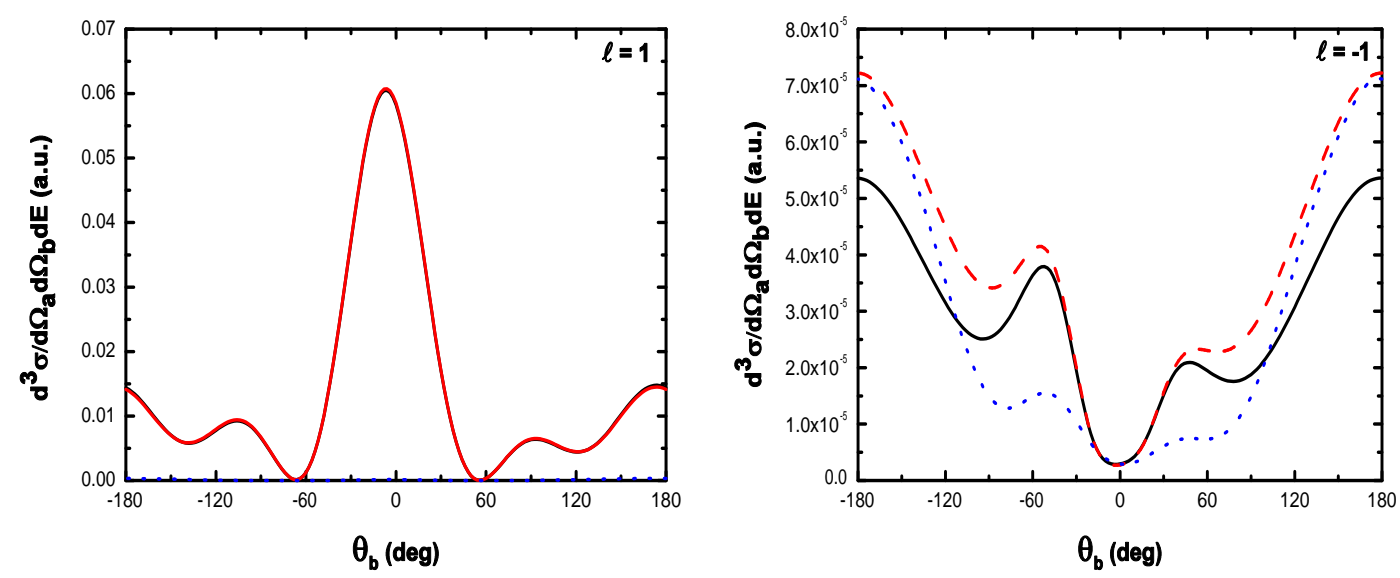

Figure 16. Same as Figure 14, but with the laser frequency $\omega=6.42 \mathrm{eV}$. 
Let us now consider the case of higher frequency lasers. The preceding discussion remains qualitatively valid as long as the condition $\omega<E_{k_{b}}$ is satisfied. This is well illustrated by our results presented in Figures 4 and 5 which display the angular distribution for the frequencies $\omega=1.17 \mathrm{eV}$ and $\omega=2.34 \mathrm{eV}$. By comparing these results, one observes that the shapes of the angular distributions are mostly the same, the main difference lying in the overall magnitude of the TDCS. Indeed, if everything else being kept fixed, the frequency is increased by a factor of 2, the electric field coupling parameter $\alpha_{0}$ is four time smaller, which correspondingly affects the magnitude of the cross sections. The high frequency regime where $\omega>E_{k_{b}}$ is satisfied leads to strong modifications of the angular distribution of the ejected electron. This is confirmed by the results given in Figure 16 for $\omega=6.42 \mathrm{eV}$ and $E_{k_{b}}=5 \mathrm{eV}$. One observes a typical splitting of the binary and recoil peaks. For the absorption of one photon $\ell=1$, the margins between SBA and FBA results are negligible, but for the case of emission $\ell=-1$, the modification is large. In fact, the margins between the SBA and FBA results are large except in the vicinity of the binary peak where the two curves coincide. As seen in Figure 16, at a given field strength, the overall magnitude of the TDCS for $\ell=-1$ is smaller by three orders of magnitude than in the case of $\ell=1$.

The results displayed in this paper show that the photon absorption processes dominate the photon emission ones, meaning that the system absorbs net energy from the laser field background. The curves for $\ell$ and $-\ell$ present similar features since $J_{-\ell}(\lambda)=(-1)^{\ell} J_{\ell}(\lambda)$. Nevertheless, the magnitudes of the cross sections for $\ell$ and $-\ell$ are different. The origin of this difference lies in terms in different expressions of $(\mathrm{e}, 2 \mathrm{e})$ scattering amplitudes other than $J_{\ell}(\lambda)$. The cross sections in Figures 12 and 13 for both geometries are different. In both cases, the oscillation structure in the results is determined by the Bessel function entering the expressions of the ionization amplitudes. When the argument $\lambda$ and the order $\ell$ are approximately equal, the value of the function $J_{\ell}(\lambda)$ diminishes rapidly. Physically, in the extreme case where $\Delta-\boldsymbol{k}_{b}$ is very small, e.g., when the nucleus is a spectator during the collision, $\lambda$ is as well very small and the laser field plays a minor role. This is because the energy absorbed by the electrons from the radiation field needs to be converted into a linear momentum via a re-scattering from the massive residual ion. Small $\left|\Delta-k_{b}\right|$ means that the re-scattering did not take place and hence the weak influence of the laser field on the outcome of the collision process. For large $\left|\Delta-k_{b}\right|$, the scattering processes take place near the nucleus and hence the probability for the electrons to experience a violative transition is generally much higher, except for $\lambda=0$ (the electric field is $\perp$ to $\Delta-\boldsymbol{k}_{b}$ ).

In Figures 17 and 18, we discuss the influence of the laser polarization orientation on the angular distribution of the ejected electron. We compare the TDCSs computed within the second Born approximation for two laser polarization directions where the polarization vector of the field is taken to be either parallel to the incident momentum $\hat{\varepsilon} / / k_{i}$ or parallel to the momentum transfer $\hat{\varepsilon} / / \Delta$. As shown in Figure 17 corresponding to $\ell=0$, the laser assisted TDCS for $\hat{\varepsilon} / / \boldsymbol{k}_{i}$ deviates form that for $\hat{\varepsilon} / / \Delta$ so long as the incident electron energy is larger than $80 \mathrm{eV}$. At $E_{k_{i}}=40 \mathrm{eV}$, the angular distribution of the ejected electron is the same for both laser polarization orientations. This can be understood as resulting from the fact that, at low incident energy, these orientations give substantially the same argument of Bessel functions entering the expression of the transition amplitudes Equations (25) and (42). The absolute magnitude of the TDCSs for the ionization of hydrogen by electron impact in the presence of a laser field increases with the increase of the incident electron energy for both laser polarization orientations $\hat{\varepsilon} / / k_{i}$ and $\hat{\varepsilon} / / \Delta$. Furthermore, the angular distribution is symmetrical with respect to the maxima of the binary and recoil peaks. With decreasing $E_{k_{i}}$, the maximum corresponding to the binary peak moves towards $\theta_{b} \simeq 0$, while the recoil peak disappears. The finding results have not been provided by a first Born treatment of laser assisted (e, 2e) collisions. 

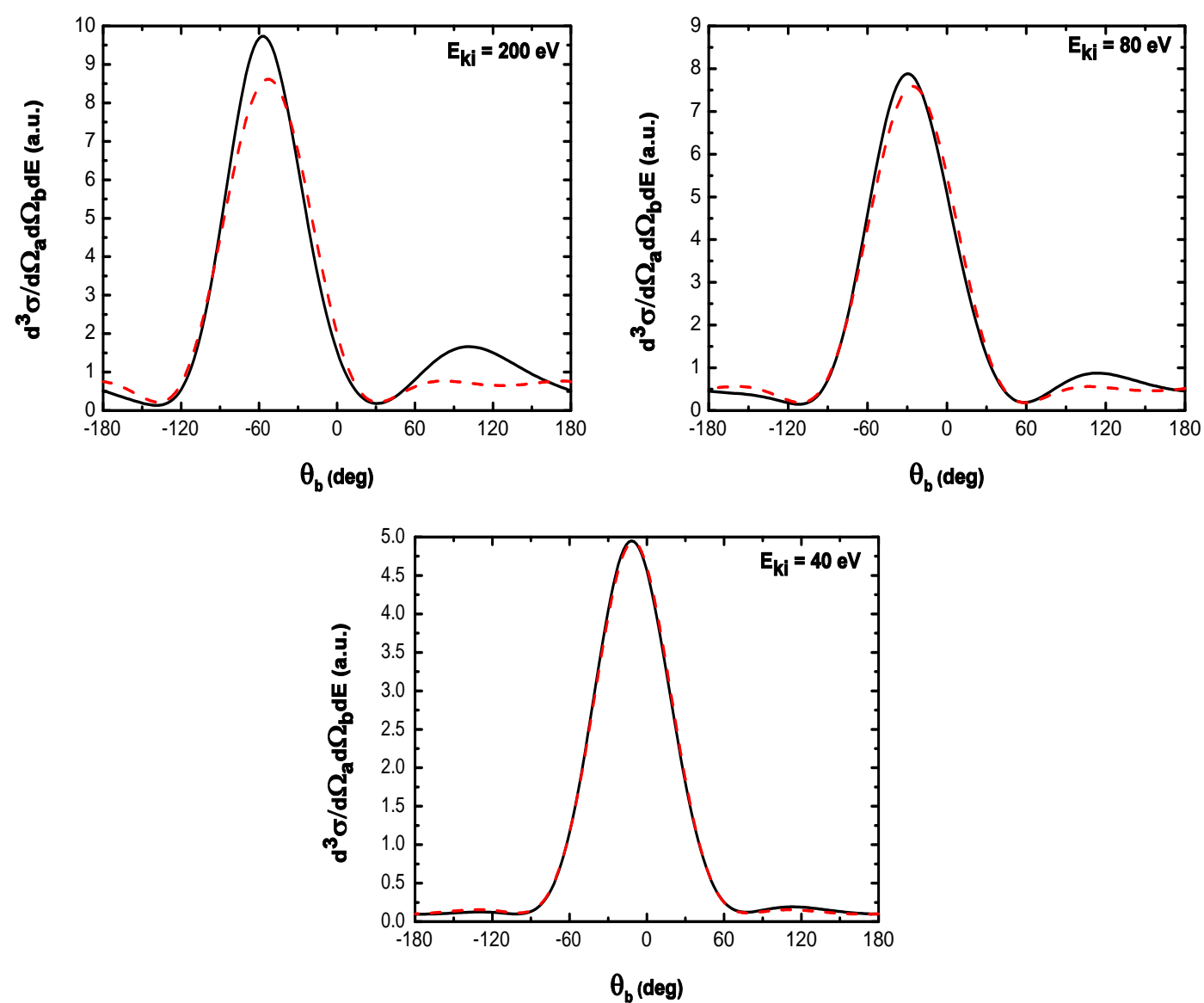

Figure 17. Triple differential cross sections corresponding to the laser-assisted electron-impact ionization process of atomic hydrogen as a function of the ejected angle $\theta_{b}$ with no net exchange of photons $(\ell=0)$ and for different values of incident electron energy $E_{k_{i}}=200,80$ and $40 \mathrm{eV}$. The laser frequency is $\omega=1.17 \mathrm{eV}$ and the electric field strength is $\mathcal{E}_{0}=10^{7} \mathrm{~V} / \mathrm{cm}$. The ejected electron energy is $E_{k_{b}}=5 \mathrm{eV}$, and the scattering angle is $\theta_{a}=4^{\circ}$. Solid lines: second Born approximation results where the laser polarization is parallel to the momentum transfer $(\hat{\varepsilon} / / \Delta)$. Dashed lines: second Born approximation results where the laser polarization is parallel to the incident momentum $\left(\hat{\varepsilon} / / \boldsymbol{k}_{i}\right)$.

We present in Figure 18 our results of ionization cross sections in the case $\ell=1$, corresponding to the net absorption of one laser photon. The angular distribution for $\hat{\varepsilon} / / \boldsymbol{k}_{i}$ differs markedly from that of $\hat{\varepsilon} / / \Delta$ when the incident energy is $\geq 100 \mathrm{eV}$ i.e., when the first Born approximation is sufficient to describe the projectile-target interaction and the higher order terms could be negligible compared to the first Born term. Indeed, the Born series will converge if the incident particle is sufficiently high so that it can not interact many times with the target and for it the interaction potential is weak enough. At high incoming energies, the choice of the orientation $\hat{\varepsilon} / / \Delta$ maximizes the cross sections, this results from the fact that this geometry maximizes the argument of the Bessel functions entering the expressions of the ionization amplitudes, as the coupling between the projectile and the laser field is maximum in this laser polarization direction. In Figure 18, the angular distribution is strongly modified as the binary and recoil peaks are split into smaller lobes with different magnitudes. The splitting of the peaks or, more precisely, the occurrence of increasing numbers of zeros in the TDCS can be traced to the behavior of the Bessel functions which appear in the expressions (25) and (42). Moreover, the magnitude of the cross section for net exchange of one photon decreases with the incident energy. The binary peak remains intense relative to the recoil peak for both laser orientations which reflects the fact that the electron-electron interaction is important and remains dominant regardless of the incident energy. The second Born approximation gives same magnitude of the cross sections and similar behavior 
of the angular distribution in the low energy regime for net exchange of one photon for both laser polarization orientations. The very small gap between the curves may be removed by taking into account second order exchange effects and higher order terms of the Born series.
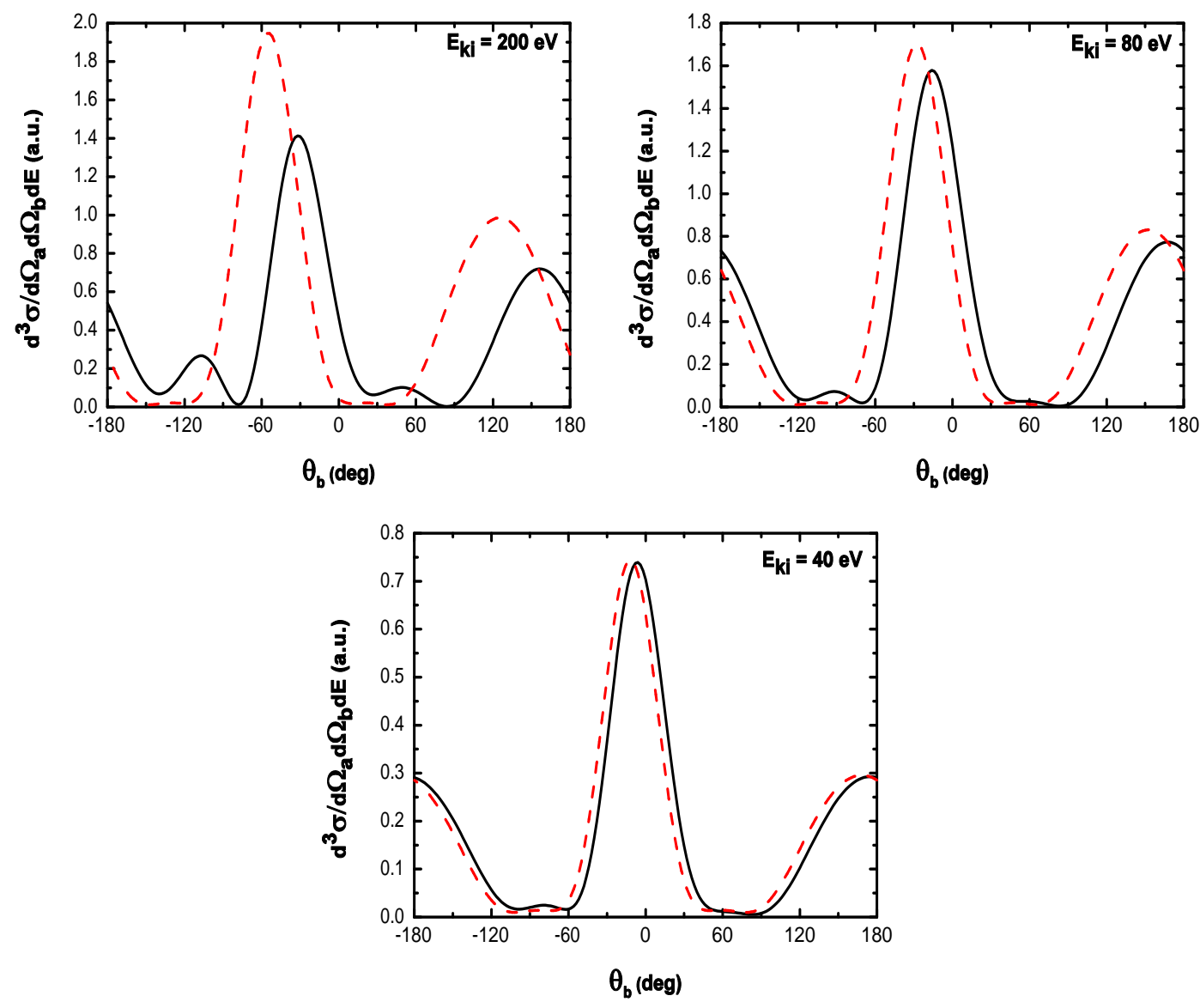

Figure 18. All the parameters are the same as Figure 17, but with the absorption of one photon $(\ell=1)$.

\section{Laser-Assisted (e, 2e) Collisions in Atomic Hydrogen in the Coplanar Symmetric Geometry}

\subsection{Theory}

In order to avoid any ambiguity in the notation of the wave vectors in the symmetric and asymmetric cases, we have chosen to change the notations for the momentums and the corresponding kinetic energies.

We shall analyze reaction Equation (2) in the coplanar symmetric geometry. In this kinematical arrangement, an electron of momentum $k_{i}$ is incident on the target, and a scattered electron of momentum $\boldsymbol{k}_{s}$ is detected in coincidence with an ejected electron of momentum $\boldsymbol{k}_{e}$, the three wave vectors $\boldsymbol{k}_{i}, \boldsymbol{k}_{s}$ and $\boldsymbol{k}_{e}$ being in the same plane. The two outgoing electrons produced on collision with the target are detected at $\theta_{s}=\theta_{e}$, at a fixed polar angle $\theta=\theta_{s}=\theta_{e}$, where $\theta_{s}$ and $\theta_{e}$ denote the scattering angle of the scattered and the ejected electrons respectively. The two outgoing electrons are indistinguishable so it is essential to include the exchange term in the cross section that is given directly from the Ochkur amplitude calculated in the absence of the laser field [85].

The magnitude of the momentum transfer is given by

$$
K=\left(k_{i}^{2}+k_{s}^{2}-2 k_{i} k_{s} \cos \theta\right)^{1 / 2},
$$

and the magnitude of the recoil momentum of the residual ion is

$$
Q=\left|2 k_{s} \cos (\theta)-k_{i}\right| \text {. }
$$


The S-matrix element for the electron-impact laser-assisted ionization of atomic hydrogen from its ground state, in the direct channel and in the FBA is then given by

$$
S_{i o n}^{B_{1}}=-i \int_{-\infty}^{+\infty} d t\left\langle\chi_{k_{s}}\left(\boldsymbol{r}_{0}, t\right) \chi_{k_{e}}\left(\boldsymbol{r}_{1}, t\right)\left|V_{d}\left(\boldsymbol{r}_{0}, \boldsymbol{r}_{1}\right)\right| \chi_{k_{i}}\left(\boldsymbol{r}_{0}, t\right) \phi_{0}\left(\boldsymbol{r}_{1}, t\right)\right\rangle
$$

with

$$
V_{d}\left(\boldsymbol{r}_{0}, \boldsymbol{r}_{1}\right)=\frac{1}{r_{01}}-\frac{1}{r_{0}}
$$

is the direct interaction potential, with $r_{01}=\left|r_{0}-r_{1}\right|$.

In these somewhat idealized conditions, the incoming and outgoing electrons are fast and their states are described in terms of Volkov functions given by Equation (8), which are solutions to the Schrödinger equation for the electron motion in a plane electromagnetic wave. On the other hand, the dressed wave function of the atomic target in the laser field corresponding to the initial bound state is given in the first-order time-dependent perturbation theory by Equation (12).

It is interesting to examine the low frequency "soft photon" limit $\left(\hbar \omega<<E_{k_{i}}\right)$ [84] of the results presented below. In this limit, the dressed ground-state wave function given by Equation (12) becomes

$$
\phi_{0}\left(\boldsymbol{r}_{1}, t\right)=e^{-i E_{0} t} e^{-i \boldsymbol{a} \cdot \boldsymbol{r}_{1}}\left[\psi_{0}\left(\boldsymbol{r}_{1}\right)-\sin (\omega t) \tilde{\psi}_{0}\left(\boldsymbol{r}_{1}\right)\right],
$$

where we have introduced the quantity

$$
\tilde{\psi}_{0}\left(\boldsymbol{r}_{1}\right)=\sum_{n} \omega_{n 0}^{-1} M_{n 0} \psi_{n}\left(\boldsymbol{r}_{1}\right) .
$$

For the case of an atomic hydrogen target, a closed form expression can be found for $\tilde{\psi}_{0}$. Denoting the hydrogen atom hamiltonian by $H=\frac{p_{1}^{2}}{2}-\frac{1}{r_{1}}$, we have

$$
\left(H-\omega_{0} \tilde{\psi}_{0}\left(\boldsymbol{r}_{1}\right)\right)=e \mathcal{E} \cdot \boldsymbol{r}_{1} \psi_{n}\left(\boldsymbol{r}_{1}\right)
$$

with $\psi_{n}\left(\boldsymbol{r}_{1}\right)=\sqrt{\pi} \exp \left(-r_{1}\right)$. The solution of this equation is readily shown to be

$$
\left(H-\omega_{0} \tilde{\psi}_{0}\left(\boldsymbol{r}_{1}\right)\right)=\sqrt{\pi} e \mathcal{E} \cdot \boldsymbol{r}_{1}\left(1+r_{1} / 2\right) \exp \left(-r_{1}\right) .
$$

This expression can be extended to the domain of higher frequencies $\left(\omega>E_{k_{e}}\right)$ by using the closure approximation [32]. We obtain

$$
\phi_{0}\left(\boldsymbol{r}_{1}, t\right) \simeq e^{-i E_{0} t} e^{-i a \cdot r_{1}}\left[\psi_{0}\left(r_{1}\right)+i \frac{\bar{\omega}_{0}}{2}\left(\frac{e^{i \omega t}}{\bar{\omega}_{0}+\omega}-\frac{e^{-i \omega t}}{\bar{\omega}_{0}-\omega}\right) \tilde{\psi}_{0}\left(\boldsymbol{r}_{1}\right)\right]
$$

with $\bar{\omega}_{0}=\frac{4}{9}$ a.u. is the average excitation energy, which gives the correct values $\alpha_{p}=4.5$ for the dipole polarisability in the closure approximation.

It is known that the S-matrix element is gauge-invariant. However, this properly can be violated if one uses approximation treatments, such as time-dependant perturbation theory when accounting for the laser field effect on electron states. In our model, we employ the length gauge, which gives more accurate perturbation results for laser-modified target states than the velocity gauge.

In the present regime, using Equations (8) and (55), we evaluate the expression

$$
\begin{aligned}
S_{\text {ion }}^{B_{1}} & =-i \int_{-\infty}^{+\infty} d t J_{\ell}(\lambda)\left\langle(2 \pi)^{-3 / 2} e^{i \boldsymbol{k}_{s} \cdot r_{0}} e^{i \boldsymbol{k}_{e} \cdot r_{1}}\left|\frac{1}{r_{01}}-\frac{1}{r_{0}}\right|(2 \pi)^{-3 / 2} e^{i k_{i} \cdot r_{0}} e^{i \boldsymbol{a}(t) \cdot \boldsymbol{r}_{1}}\right. \\
& \left.\times\left[\psi_{0}\left(r_{1}\right)+i \frac{\bar{\omega}_{0}}{2}\left(\frac{e^{i \omega t}}{\bar{\omega}_{0}+\omega}-\frac{e^{-i \omega t}}{\bar{\omega}_{0}-\omega}\right) \tilde{\psi}_{0}\left(\boldsymbol{r}_{1}\right)\right]\right\rangle
\end{aligned}
$$


where we have used the generating function of Bessel functions defined by

$$
\exp (i \lambda \sin \theta)=\sum_{\ell=-\infty}^{\ell=+\infty} J_{\ell}(\lambda) \exp (i \ell \theta)
$$

using a similar relationship to the gauge factor

$$
e^{a(t) \cdot r_{1}}=\sum_{N=-\infty}^{N=+\infty}(-i)^{N} e^{-i N \omega t} J_{N}(z)
$$

with $z=\mathcal{E}_{0} \cdot r_{1} / \omega$, by performing the integration on the time variable in Equation (56) and redefining judiciously summing indices we obtain

$$
S_{i o n}^{B_{1}}=-(2 \pi)^{-1} i \sum_{\ell=-\infty}^{\ell=+\infty} \delta\left(E_{\boldsymbol{k}_{s}}+E_{\boldsymbol{k}_{e}}-E_{\boldsymbol{k}_{i}}-E_{0}-\ell \omega\right) f_{\text {ion }}^{B_{1} \ell}\left(\boldsymbol{k}_{s}, \boldsymbol{k}_{e}\right)
$$

where $f_{i o n}^{B_{1}, \ell}\left(\boldsymbol{k}_{s}, \boldsymbol{k}_{e}\right)$ is the first-Born approximation to $(e, 2 e)$ scattering amplitude with the transfer of $\ell$ photons. This quantity is given by

$$
f_{\text {ion }}^{B_{1}, \ell}\left(\boldsymbol{k}_{s}, \boldsymbol{k}_{e}\right)=f_{1}+f_{2}
$$

with

$$
f_{1}=-(2 \pi)^{-5 / 2} \sum_{N=-\infty}^{N=+\infty} J_{L-N}(\lambda)\left\langle e^{i \boldsymbol{k}_{s} \cdot r_{0}} e^{i k_{e} \cdot r_{1}}\left|J_{N}(z)\left(\frac{1}{r_{01}}-\frac{1}{r_{0}}\right)\right| e^{i k_{i} \cdot r_{0}} \psi_{0}\left(\boldsymbol{r}_{1}\right)\right\rangle
$$

and

$$
\begin{aligned}
f_{2}= & -(2 \pi)^{-5 / 2} \sum_{N=-\infty}^{N=+\infty} i \frac{\bar{\omega}_{0}}{2}\left(\frac{J_{L-N-1}(\lambda)}{\bar{\omega}_{0}-\omega}-\frac{J_{L-N+1}(\lambda)}{\bar{\omega}_{0}+\omega}\right) \\
& \times\left\langle e^{i \boldsymbol{k}_{s} \cdot r_{0}} e^{i \boldsymbol{k}_{e} \cdot r_{1}}\left|J_{N}(z)\left(\frac{1}{r_{01}}-\frac{1}{r_{0}}\right)\right| e^{i k_{i} \cdot r_{0}} \widetilde{\psi}_{0}\left(\boldsymbol{r}_{1}\right)\right\rangle .
\end{aligned}
$$

Here $\lambda=\left(\Delta-\boldsymbol{k}_{e}\right) \cdot \alpha_{0}$ and $\Delta=\boldsymbol{k}_{i}-\boldsymbol{k}_{s}$ is the momentum transfer of the collision. In each of these two expressions, the integral over the variable $r_{0}$ is easily accomplished using the Bethe formula [58]. The integral over the variable $\boldsymbol{r}_{1}$ is more problematic because of the presence of the Bessel function. However, by using the integral representation

$$
J_{N}(z)=\frac{(i)^{-N}}{\pi} \int_{0}^{\pi} e^{i z \cos (\alpha)} \cos (N \alpha) d \alpha,
$$

and posing $\zeta=\cos (\alpha) \mathcal{E}_{0} \omega^{-1}$, we may write the expressions (61) and (62) to the following forms

$$
f_{1}=-2 \frac{K^{-2}}{\pi} \sum_{N=-\infty}^{N=+\infty}(-1)^{N} J_{N}(z) \int_{0}^{\pi} d \alpha \cos (N \alpha)\left[\tilde{M}\left(\boldsymbol{K}+\zeta, \boldsymbol{k}_{e}\right)-\tilde{M}\left(\zeta, \boldsymbol{k}_{e}\right)\right],
$$

and

$$
\begin{gathered}
f_{2}=-i \frac{K^{-2}}{\pi} \sum_{N=-\infty}^{N=+\infty}(-1)^{N} \bar{\omega}_{0}\left(\frac{J_{L-N-1}(\lambda)}{\bar{\omega}_{0}-\omega}-\frac{J_{L-N+1}(\lambda)}{\bar{\omega}_{0}+\omega}\right) \\
\times \quad \int_{0}^{\pi} d \alpha \cos (N \alpha)\left[\tilde{N}\left(\boldsymbol{K}+\zeta, \boldsymbol{k}_{e}, \mathcal{E}_{0}\right)-\tilde{N}\left(\zeta, \boldsymbol{k}_{e}, \mathcal{E}_{0}\right)\right] .
\end{gathered}
$$


with

$$
\tilde{M}\left(\boldsymbol{Q}, \boldsymbol{k}_{e}\right)=(2 \pi)^{-3 / 2}<e^{i \boldsymbol{k}_{e} \cdot \boldsymbol{r}_{1}}\left|e^{i \boldsymbol{Q} \cdot \boldsymbol{r}}\right| \psi_{0}\left(\boldsymbol{r}_{1}\right)>,
$$

and

$$
\tilde{N}\left(\boldsymbol{Q}, \boldsymbol{k}_{e}, \mathcal{E}_{0}\right)=(2 \pi)^{-3 / 2}<e^{i \boldsymbol{k}_{e} \cdot \boldsymbol{r}_{1}}\left|e^{i \boldsymbol{Q} . \mathbf{r}}\right| \tilde{\psi}_{0}\left(\boldsymbol{r}_{1}\right)>.
$$

In the coplanar symmetric geometry, the two outgoing electrons are indistinguishable, which requires the inclusion of the exchange effects between the projectile and the target electrons in the calculation of the cross sections. The contribution the exchange scattering to laser-assisted (e, 2e) collisions leads to some conceptual difficulties but would not significantly alter the results of the present discussion. We have considered in the present work only the leading term, $g_{i o n}^{B_{1}, \ell}$, of the exchange amplitude for laser-assisted ionization with the transfer of $\ell$ photons. Thus, the First-Born TDCS, with the transfer of $\ell$ photons, is given by

$$
\frac{d^{3} \sigma_{i o n}^{B_{1}, \ell}}{d \Omega_{s} d \Omega_{e} d E}=\frac{k_{s} k_{e}}{k_{i}}\left(\frac{1}{4}\left|f_{i o n}^{B_{1}, \ell}+g_{\text {ion }}^{\ell}\right|^{2}+\frac{3}{4}\left|f_{i o n}^{B_{1}, \ell}-g_{\text {ion }}^{\ell}\right|^{2}\right)
$$

where the exchange amplitude is approximated by its dominant part coming from the first Born term $g_{\text {ion }}^{\ell}$ given by [85]

$$
g_{\text {ion }}^{\ell}(\Delta) \simeq J_{\ell}(\lambda) g_{\text {ion }}^{\text {Och }} .
$$

with $g_{\text {ion }}^{\text {Och }}$ is the exchange amplitude in the Ochkur approximation calculated in the absence of the field.

\subsection{Results and Discussion}

\subsubsection{Triple Differential Cross Sections of Laser-Assisted (e, 2e) Collisions at $E_{k_{i}}=500 \mathrm{eV}$}

In this section, we present and analyze our finding results of the TDCS of the dynamics of (e, 2e) collisions in the coplanar symmetric geometry in the presence of a linearly polarized and single mode laser field. Our calculations focus on the case of atomic hydrogen. In these somewhat idealized conditions, the laser effects on the $(\mathrm{e}, 2 \mathrm{e})$ reaction depend on the following laser parameters: the laser photon energy $\omega$, the field strength $\mathcal{E}_{0}$ and the polarization direction $\hat{\varepsilon}=\mathcal{E}_{0} / \mathcal{E}_{0}$. We shall discuss the influence of these parameters by keeping the polarization vector $\hat{\varepsilon}$ of the field parallel to the momentum transfer $\Delta$. The reason we adopt this geometry is that the angular part of the scattering amplitude may be simplified and, what is more important, because for small momentum transfers, when approximately $k_{i} \| \Delta$, the coupling of the colliding system with the field has its minimal value for $\hat{\varepsilon} \| \boldsymbol{k}_{i}$, and its maximal value for $\hat{\varepsilon} \| \Delta$ [76]. In a realistic experiment, the choice of the geometry $\hat{\varepsilon} \| \Delta$ causes inconveniences because of the intensity of rotating the laser beam for each $\ell$ and scattering angle $\theta$. Moreover, in the case of small-frequency and small-momentum transfer collisions, the results referring to the geometry $\hat{\varepsilon} \| \Delta$ should be very close to those obtained for $\hat{\varepsilon} \perp \Delta$.

We want to stress the importance of laser field effects in the case of another interesting (e, 2e) kinematical arrangement, namely the coplanar symmetric energy-sharing geometry at small scattering angles. We note that the momentum transfer $\Delta$ is never small in this geometry, in contrast with the coplanar asymmetric geometry (Ehrhardt asymmetric kinematics). On the other hand, the values of the recoil momentum of the residual ion $Q=\boldsymbol{k}_{i}-\boldsymbol{k}_{s}-\boldsymbol{k}_{e}$ remain moderate for scattering angles $\theta \leq 60^{\circ}$ (with $Q \simeq 0$ when $\theta \simeq 45^{\circ}$ for $\ell=0$ and $E_{i}=500 \mathrm{eV}$ ), while at large scattering angles both $\Delta$ and $Q$ are large. In the absence of the laser field, the angular domain $\theta \leq 70^{\circ}$ may be called the $(\mathrm{e}, 2 \mathrm{e})$ spectroscopy region [86], since in this angular range the momentum density distribution of the target electrons may be obtained (see Reference [86]). In this paper, we are mainly interested to this small-angle region, i.e., $Q$ is relatively small for performing $(\mathrm{e}, 2 \mathrm{e})$ spectroscopy studies. 
In Figure 19, we give the TDCS's corresponding to the laser-assisted electron-impact ionization of atomic hydrogen as a function of $\theta=\theta_{e}=\theta_{s}$. The polarization vector of the field $\hat{\varepsilon}$ is set parallel to the momentum transfer $\Delta$. The complete results obtained by using the ionization amplitude Equation (60) in the FBA are compared with those obtained by neglecting the dressing of the target and with the field-free results. We have chosen to address the case of laser-assisted collisions with no photons exchanged between the projectile-atom system and the laser field, i.e., the energies of the outgoing electrons are the same as those obtained in the field-free case. The modifications of the cross sections directly reflect the role of the dressing of the projectile-target system by the external field. As shown in Figure 19, the laser-assisted TDCS deviates little from the field-free one, so long as the laser field strength is not larger than $\sim 10^{7} \mathrm{~V} / \mathrm{cm}\left(\sim 2 \times 10^{-3}\right.$ a.u.). The influence of changing the laser field strength, everything else being kept fixed, on the angular distribution reflects the role of the dressing of the projectile-target system by the external laser field. As shown in the Figure 19a,b, the laser-assisted TDCS deviates very little from the field-free one, so long as the laser field strength is not larger than $\sim 10^{7} \mathrm{~V} / \mathrm{cm}\left(\sim 1.32 \times 10^{11} \mathrm{~W} / \mathrm{cm}^{2}\right)$. At higher intensity however, significant changes take place, as shown in Figure $19 \mathrm{~d}$, which are obtained for a field strength $\mathcal{E}_{0}=10^{8} \mathrm{~V} / \mathrm{cm}$. The angular distribution is then strongly modified and several interesting points characteristic of this class of laser-assisted collisions can be made at the (e, 2e) spectroscopy region.

(i) For laser field strengths lower than $10^{7} \mathrm{~V} / \mathrm{cm}$, and for large values of $\Delta$, one can model the $(\mathrm{e}, 2 \mathrm{e})$ reaction as an electron-electron collision, with $(-Q)$ being the momentum of the struck electron before the collision;

(ii) The magnitude of the cross section for no net exchange of photons is significantly smaller than in the field-free case. This results from the fact that the laser itself does not contribute to the ionization process except for $\theta \simeq 44^{\circ}$ which corresponds to the case where the recoil momentum of the ionized target $\left(H^{+}\right.$in the present case) is zero, i.e., when the TDCS is maximum. For $\theta<60^{\circ}$ and $\theta \neq 44^{\circ}$ the laser field redistributes the ejected electrons in new channels associated to indices $\ell \neq 0$ in the energy conservation relation $\left[E_{k_{i}}+E_{0}+\ell \omega=E_{k_{s}}+E_{k_{e}}\right]$, which are accessible in the "dressed" continuum of the atomic target. A simplified description of this process is that of two-step processes in the course of which the atomic electron is first brought into the continuum as a result of the collision, and then exchanges photons with the laser field [87]. When the laser intensity increases, an oscillatory phenomenon is observed which is due to the interference between the terms constituting the dominant electronic amplitude $f_{1}$ given by Equation (61) and the change of sign of the argument of the Bessel function (see below);

(iii) Figure 19 indicates that, for $\ell=0$, the dressing of the target states does not play a dominant role in the physics of the process. In other words, this result shows that the term $f_{1}$ (Equation (61)) is dominant in the transition amplitude (Equation (60)). This is a signature of coplanar symmetric (e, 2e) collisions in the presence of the laser field in the $(e, 2 e)$ spectroscopy region.

We also notice significant changes in the overall magnitude of the cross section when varying the field strength intensity for $\ell=0$, where the amplitude is dominated by the Bessel function $J_{0}$. Although this trend is expected for laser-assisted processes, it is by no means easily deduced from the structure of the transition amplitudes (Equations (64) and (65)). The magnitude of the argument of the Bessel function is linked, in turn, to the electron-field coupling, the strength of which depends on the laser intensity and frequency thought the parameter $\alpha_{0}=\mathcal{E}_{0} / \omega^{2}$. In fact, the magnitude of the argument of the Bessel functions $J_{\ell}$ is directly proportional to $\mathcal{E}_{0}$, contrarily to $J_{0}$ which decreases from unity when $|\lambda|$ increases, the other functions $J_{\ell}(\lambda)$ start to increase from zero and grow as $|\lambda|$. Although this argument cannot account for the details of the variations of the TDCS with the laser intensity it provides a fair estimate of the overall changes observed. 

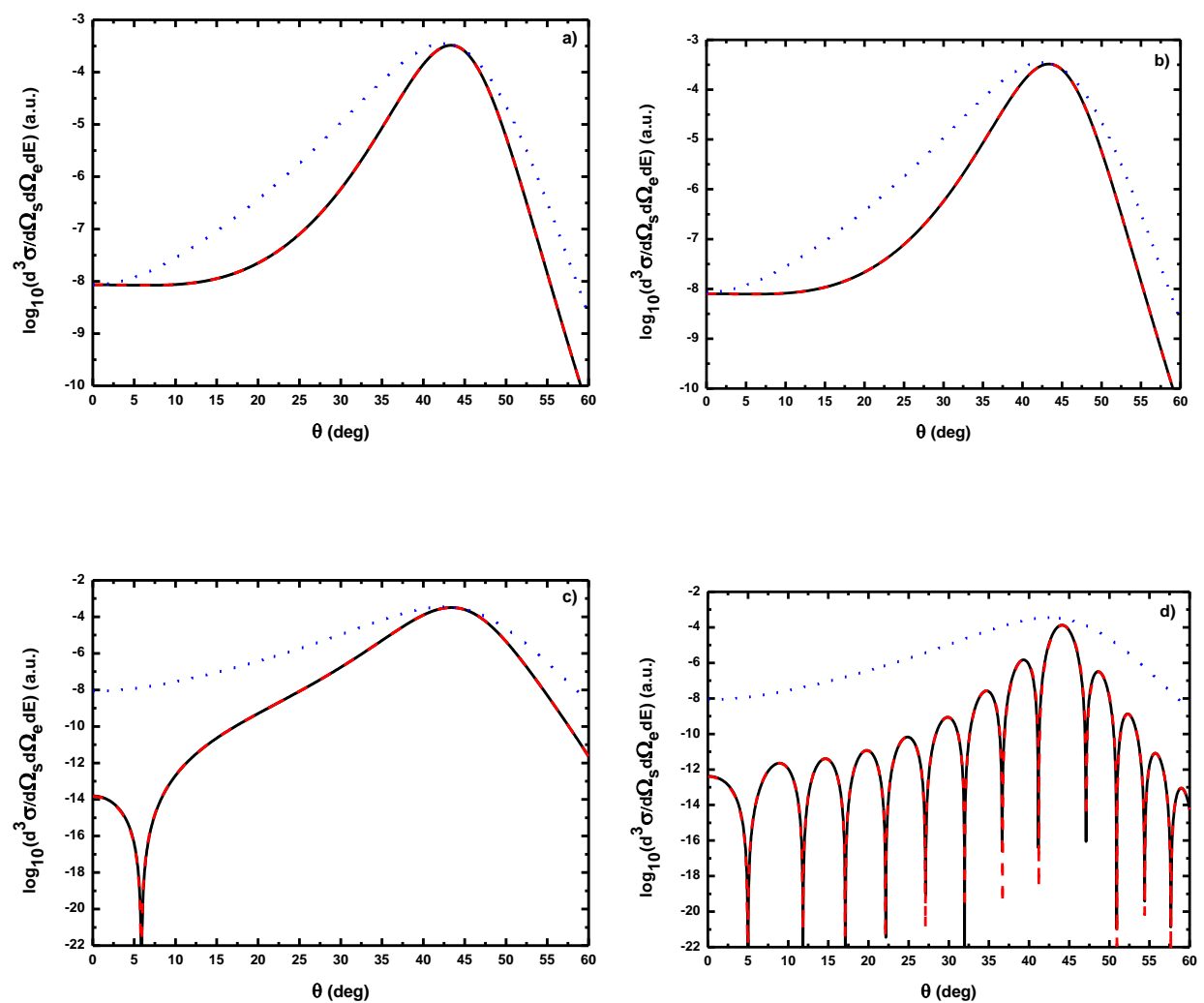

Figure 19. (Color online) Triple differential cross sections corresponding to laser-assisted electron-impact ionization of atomic hydrogen in the ground state for the case of a coplanar symmetric geometry as a function of the scattering angle $\theta=\theta_{s}=\theta_{e}$ with no net exchange of photons $(\ell=0)$. The incident electron energy is $E_{k_{i}}=500 \mathrm{eV}$, and the laser photon energy is $\omega=1.17 \mathrm{eV}$. The laser polarization vector is taken to be parallel to the momentum transfer $\Delta$. Solid lines: First Born approximation results; dashed line: results obtained by neglecting the dressing of the target by the laser field; dotted lines: field-free results. (a) The electric field strength is $\mathcal{E}_{0}=10^{5} \mathrm{~V} / \mathrm{cm}$ which corresponds to a laser beam intensity of $I=1.32 \times 10^{7} \mathrm{~W} / \mathrm{cm}^{2}$; (b) $\mathcal{E}_{0}=10^{6} \mathrm{~V} / \mathrm{cm}\left(I=1.32 \times 10^{9} \mathrm{~W} / \mathrm{cm}^{2}\right)$; (c) $\mathcal{E}_{0}=10^{7} \mathrm{~V} / \mathrm{cm}\left(I=1.32 \times 10^{11} \mathrm{~W} / \mathrm{cm}^{2}\right)$ and (d) $\mathcal{E}_{0}=10^{8} \mathrm{~V} / \mathrm{cm}\left(I=1.32 \times 10^{13} \mathrm{~W} / \mathrm{cm}^{2}\right)$.

Figure 20 shows TDCS in the case $\ell=1$ corresponding to the net absorption of one photon. The complete results obtained by using the ionization amplitude Equation (60) in the FBA are compared with those obtained by neglecting the dressing of the atomic target (the electronic amplitude $f_{1}$ ). It is interesting to note that at low frequencies, the target dressing is less important and one can model the atom by a structureless center of force. Note that the target distortion by the laser field should be fully taken into account at high laser photon energies (see below). Such a distorted atom also acts on the projectile by a long-range dipole potential $\left(1 / r^{0}\right)$, which requires a non-perturbative treatment of the laser-atom interaction at low incident electron energies. The long-range dipole potential affects mainly the distant collisions, which contribute when the energies of the primary electron are weak and the second-Born approximation is needed. Another interesting point is the fact that the difference between the overall magnitude of the cross sections in FBA and without dressing remain practically zero with the increase of the electric field strength associated to the laser field. The results, at low frequencies, show a distinct two peaks structure. To the right of Figure 20a-c is attributed to the electron-electron interaction (term on $1 / \boldsymbol{r}_{1}$ ) while the left peak is governed by the attraction between the electron and the nucleus (term on $1 / \boldsymbol{r}_{0}$ ). At higher field strengths (see Figure 20d), every other laser parameter being fixed, one explores more zeros of the angular distribution when varying the scattering angle and the TDCS increases. These rapid oscillations reflect the highly non-perturbative nature of the interaction 
of the laser field with the free electron. This oscillatory phenomenon can be explained by the changes of the Bessel function, i.e., the sign of the argument of the Bessel function changes depending on the sign of the summation over $\mathrm{N}$ coming from Equation (61). This behavior can be traced back to the fact that the argument of the Bessel functions, entering the expressions of the amplitudes Equations (61) and (62), grows with $\mathcal{E}_{0}$ and varies with the scattering angle. Accordingly, at higher field strengths, every other laser parameter being fixed, one explores more zeros of the Bessel functions when varying the scattering angle. This clearly gives rise to the observed increase in the number of minima. This can be explained physically by the presence of the laser field which breaks the symmetry of the angular distribution. The observed symmetry breaking results from the fact that the orientation of the laser polarization chosen is parallel to the incident momentum. We see that, even for intense laser fields, the dressing effects of the target are somewhat insignificant at low frequencies. Moreover, our results present an absolute minimum corresponding to the zero of the electronic term (term in brackets in Equation (61)) which is dominant in low frequencies. This minimum, located around $\theta=44^{\circ}$, corresponds to the compensation between electron-electron and electron-nucleus interactions. In fact, at $\theta=44^{\circ}$ the incident electron, after being dressed by the laser field, collides with the atom without effect, i.e., that the atom becomes transparent for such electron and then the target can be modeled by a structureless center of force. This situation corresponds to the case where the scattered and ejected electrons exit perpendicular with respect to each other.
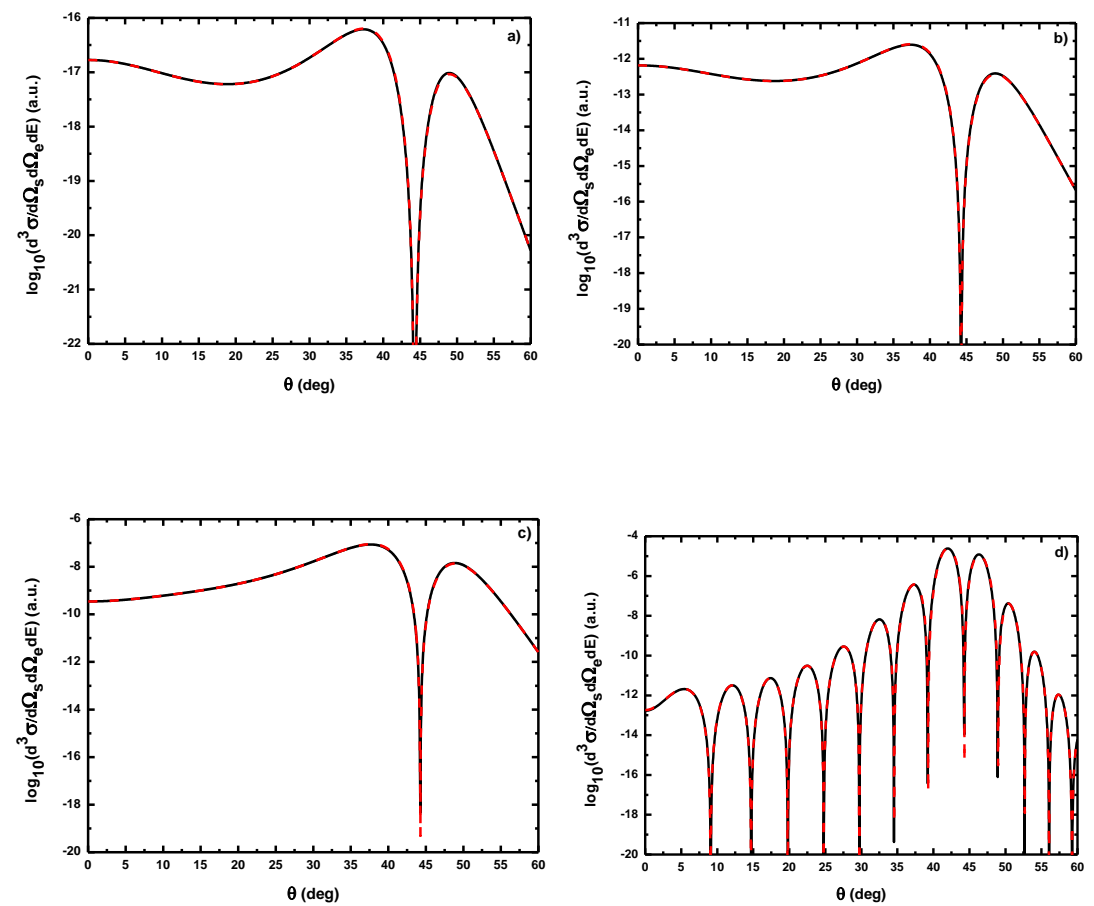

Figure 20. (Color online) All the parameters are the same as Figure 19, but with the absorption of one photon $(\ell=1)$.

The role of the laser frequency can be appreciated by comparing Figures 21 and 22 . We have chosen some frequencies which are typical of laser sources currently operated, namely the near infrared $[\omega=1.17 \mathrm{eV} \mathrm{Nd}$ :YAG (Yttrium Aluminum Garnet) laser, Figure 22a], first harmonic of Nd:YAG laser $[\omega=2.34 \mathrm{eV}$, Figure 21a,b], UV [ $\omega=4.95 \mathrm{eV} \mathrm{Kr} * \mathrm{~F}$ laser, Figure 21b], UV [ $\omega=6.42 \mathrm{eV}$ Ar*F laser, Figures 21c and 22c] and first harmonic of $\mathrm{Kr}^{*} \mathrm{~F}$ excimer laser [ $\omega=9.9 \mathrm{eV}$, Figure 21d]. The most remarkable feature of the frequency dependence of the TDCS is the fact that the magnitude of the cross section decreases when the laser frequency increases. One observes that the laser-assisted cross section does vary as an inverse power comprised between 2 and 3 of the frequency, a feature which is reminiscent of the infrared divergence of quantum electrodynamics which accounts for the divergence 
of the bremsstrahlung and free-free cross sections in the soft-photon limit [75]. The breakdown of the approximation neglecting dressing effects at higher frequencies is clearly shown in Figure 21c,d. In fact, at relatively high laser frequencies, the dressing of the target states plays a dominant role in the physics of the process, a result which implies that the contribution of the atomic amplitude $f_{2}$ becomes significant in the transition amplitude. The effects of the internal structure of the atomic target is specially significant in the interval of scattering angles $\left[32^{\circ}, 55^{\circ}\right]$. In the symmetric coplanar geometry, this demonstrates that low frequency lasers can more effectively dress continuum states than bound atomic states. It shows also that Volkov waves correctly approximate the wave function of a continuum electron in a low frequency field. Furthermore, we observe that the shape of the angular distribution remains the same. Thus, we can approximate our treatment on two areas, namely

$\theta \in\left[32^{\circ}, 55^{\circ}\right]$, the difference in shape and magnitude between FBA results and those obtained by neglecting the target dressing, at high laser frequencies, is traced to the role played by the explicit introduction of the effects of the atomic target internal structure;

$\theta \in\left[0^{\circ}, 32^{\circ}[\cup] 55^{\circ}, 70^{\circ}\right]$, the electronic term given by Equation (61) is sufficient in such process so that the atomic target can be described by unperturbed wave function.
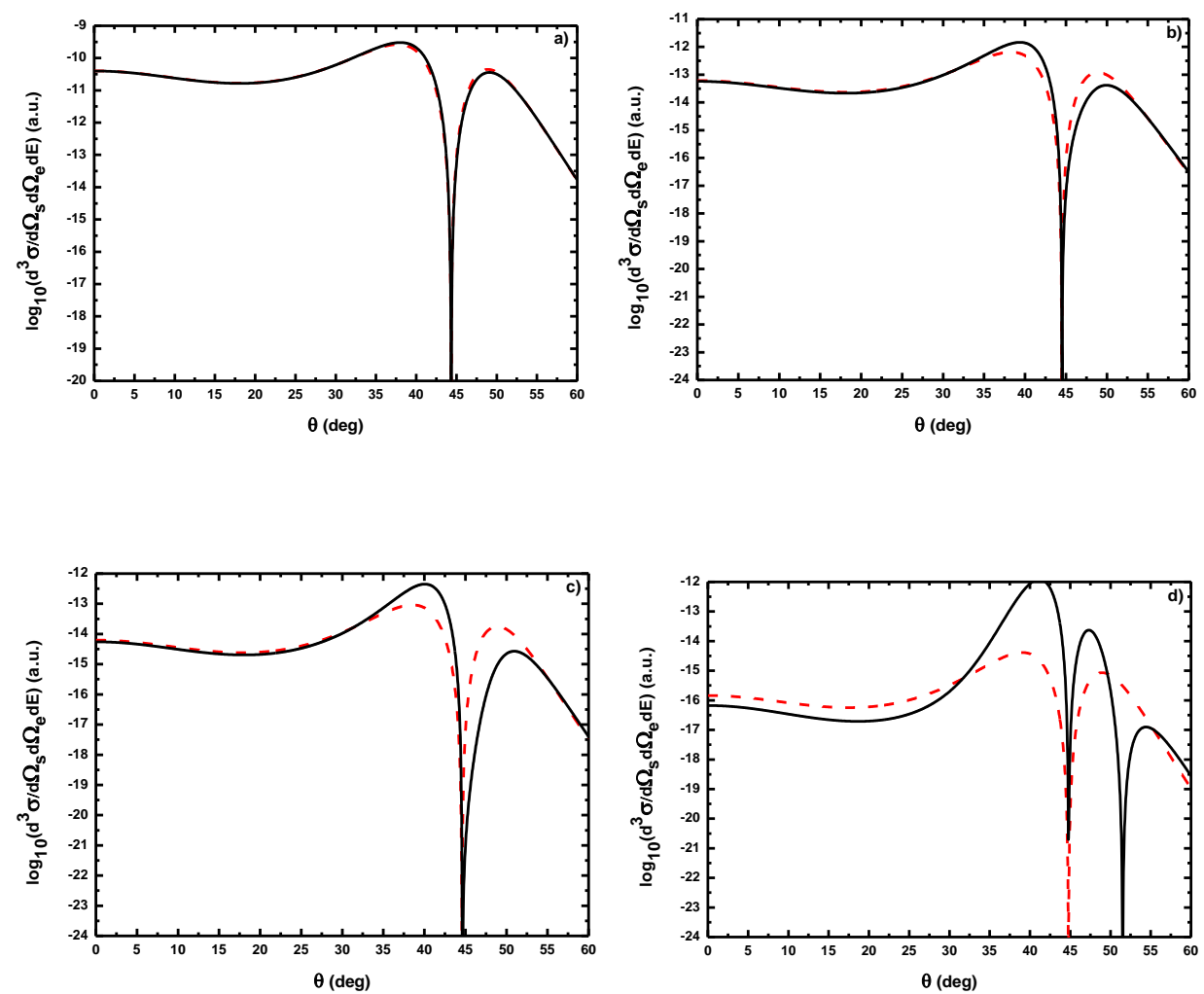

Figure 21. Triple differential cross sections corresponding to laser-assisted electron-impact ionization of atomic hydrogen in the ground state for the case of a coplanar symmetric geometry as a function of scattering angle $\theta=\theta_{s}=\theta_{e}$ for the absorption of one photon $(\ell=1)$. The incident electron energy is $E_{k_{i}}=500 \mathrm{eV}$, and the electric field strength is $\mathcal{E}_{0}=10^{7} \mathrm{~V} / \mathrm{cm}$. The laser polarization vector is taken to be parallel to the transfer momentum $\Delta$. Solid lines: First Born approximation results. Dashed line: results obtained by neglecting the dressing of the target by the laser field. (a) The laser photon energy is $\omega=2.347 \mathrm{eV}$; (b) $\omega=4.95 \mathrm{eV}$; (c) $\omega=6.42 \mathrm{eV}$ and (d) $\omega=9.9 \mathrm{eV}$.

Another consequence of increasing the laser photon energy is that the electron-electron interaction becomes more important than the electron-nucleus attraction. The peak corresponding to the electron-electron interaction increases by an amount that is the same as that corresponding to the decrease of the peak associated to the electron-proton interaction around the deep minimum at $\theta \simeq 44^{\circ}$ 
at low frequencies $(\omega<9 \mathrm{eV})$. In Figure $21 \mathrm{~d}$, the minima corresponding to the full calculation taking into account dressing effects is due to the destructive interference between the electronic Equation (61) and atomic Equation (62) amplitudes near $\theta=52^{\circ}$, i.e., the electronic and the atomic amplitudes are varying in opposite directions when the momentum transfer increases.

The set of Figures 21 and 22 also clearly shows the inadequacy of the simplified approach in which one neglects the dressing of the atomic target states by the field. Comparing again our results given by Equation (68) with those obtained with the help of Equation (61), one observes that, although the shape of the angular distribution is fairly well reproduced, the overall magnitude of the TDCS is significantly underestimated contrarily to the case $\ell=0$ discussed in Figure 19. This clearly shows that the collision dynamics, in the symmetric coplanar geometry, is strongly affected by laser photon energy even at moderate laser intensities.
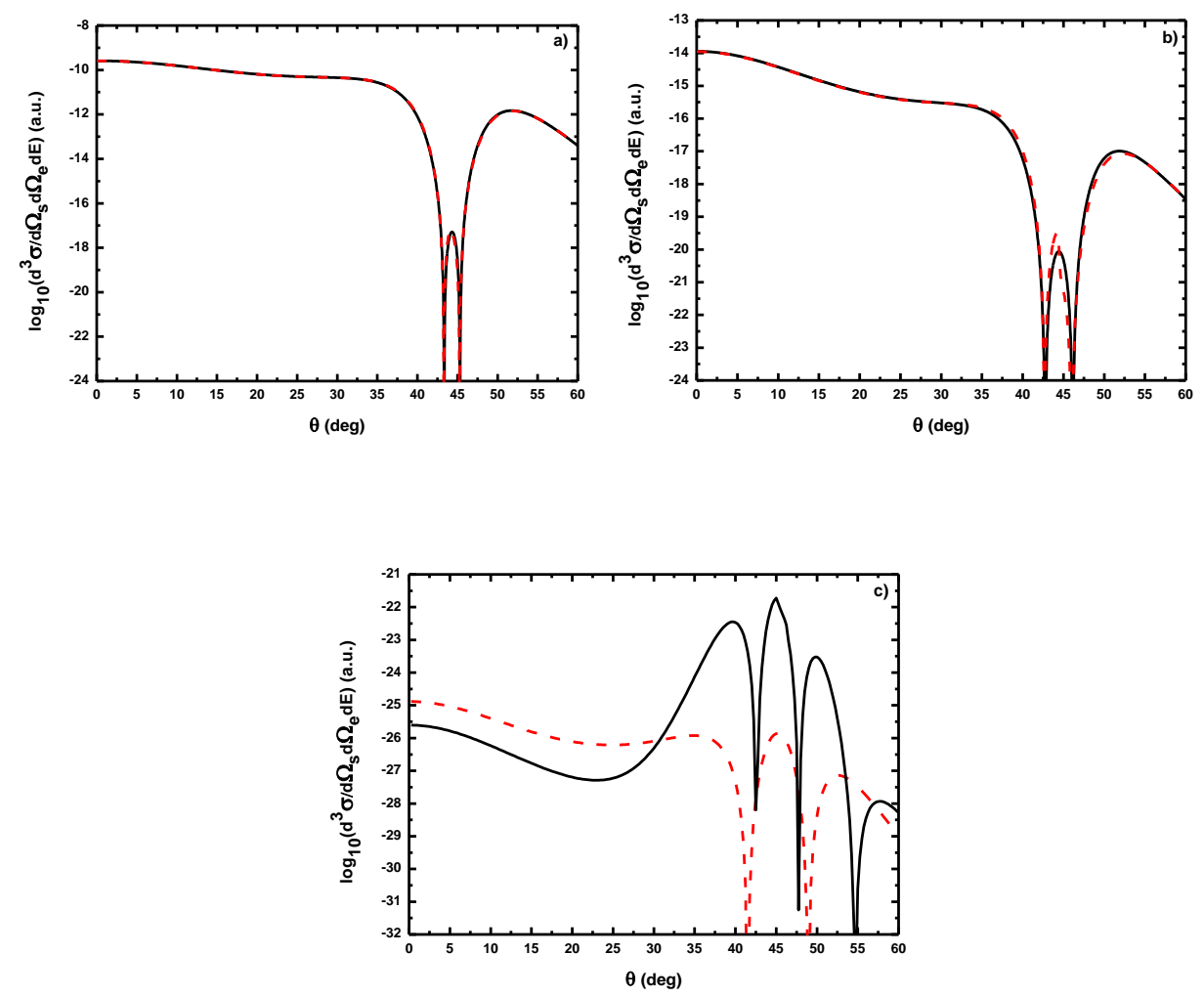

Figure 22. (Color online) Triple differential cross sections corresponding to the laser-assisted electron-impact ionization of atomic hydrogen in the ground state for the case of a coplanar symmetric geometry as a function of scattering angle $\theta=\theta_{s}=\theta_{e}$ for the absorption of two photons $(\ell=2)$. The incident electron energy is $E_{k_{i}}=500 \mathrm{eV}$, and the electric field strength is $\mathcal{E}_{0}=10^{7} \mathrm{~V} / \mathrm{cm}$. The laser polarization vector is taken to be parallel to the transfer momentum $\Delta$. Solid lines: First Born approximation results. Dashed line: results obtained by neglecting the dressing of the target by the laser field. (a) The laser photon energy is $\omega=1.17 \mathrm{eV}$; (b) $\omega=2.34 \mathrm{eV}$; and (c) $\omega=6.42 \mathrm{eV}$.

These tendencies are even amplified when turning to higher net number of exchanged photons, as shown in Figure 22, where TDCS are displayed for the case $\ell=2$. The modifications of the angular distribution are observed, the number positions of the nodes being notably changed when increasing the number of photons exchanged between the external laser field and the electron-atom system. The most remarkable fact, however, is the important changes of magnitude of TDCS when varying the number of photons exchanged. In fact, the overall magnitude of TDCS, at a given field strength becomes smaller when increasing the frequency. Again, this can be ascribed to the form of the coupling parameter which exhibits an $\omega^{-2}$ dependence. Another interesting point is the fact that, again, the simplified model used by neglecting the target dressing can not properly 
reproduce our more complete calculations at high laser frequencies. In Figure 22, the TDCS displays two distinct minima. Both minima correspond to values of the scattering angle for which the cross section is actually zero, nevertheless the origin of these zeros is different for each case. The first one amount for the full calculation appears at angles for which the TDCS vanishes, i.e., $f_{1}+f_{2}=0$. This results from a destructive interference between the electronic and atomic terms. The second kind of minimum appears at angles for which the electronic amplitude vanishes $\left(f_{1}=0\right)$. This results from a destructive interference between the electronic terms. These two kind of minima coincide when the electronic amplitude is dominant (the dressing effect is neglected: $f_{2}$ is negligible compared to $f_{1}$ at low laser photon energies). The presence of the relative maximum between two successive minima corresponding to $f_{1}$ and $f_{1}+f_{2}$ comes from the fact that the argument of the Bessel function changes its sign. It is also important to note that these amplitudes are sensitive to the number of laser photons exchanged between the external laser field and the electron-atom system near $\theta=44^{\circ}$.

\subsubsection{Triple Differential Cross Sections of Laser-Assisted (e, 2e) Collisions at $E_{k_{i}}=250 \mathrm{eV}$}

In what follows, we discuss the influence of the laser parameters (the number of exchanged photons $\ell$, the field strength $\mathcal{E}_{0}$, the photon energy $\omega$ and the polarization direction $\hat{\varepsilon}$ ) by keeping the polarization direction parallel or perpendicular to $\mathbf{k}_{i}$ and studying their dependence on the angular distribution. The incident electron energy chosen is $250 \mathrm{eV}$. It is convenient to note that, when the incident electron is fast, the magnitude $\Delta$ of the momentum transfer is never small in a symmetric geometry (see Table 1), the magnitude of the recoil momentum of the residual ion can be either small (or moderate) or it can become large. This is quite clear in Table 1, where the values of $Q$ remain small or moderate for scattering angles $\theta \leq 70^{\circ}$ while for $\theta>70^{\circ}$ the magnitude of the recoil momentum becomes large. In the absence of the external laser field, the value of $\Delta$ for the symmetric (e, 2e) reactions is relatively small and then is convenient for performing $(\mathrm{e}, 2 \mathrm{e})$ spectroscopy studies [88]. On the other hand, the case of symmetric $(e, 2 e)$ reactions for which $\Delta$ is large has been analyzed subsequently. Furthermore, when the magnitude of the recoil momentum of the residual ion is small, while the magnitude of the momentum transfer remains large one has impulse-type collisions, from which the electron momentum density distribution of the target atom was obtained. According to Table 1 and what was said above about the $(\mathrm{e}, 2 \mathrm{e})$ reaction in the absence of the laser field, we can extend the analysis to the case in the presence of the external laser field in the angular domains where $\Delta$ is large and $Q$ is small or moderate, i.e., in the $(e, 2 e)$ spectroscopy region.

Table 1. The magnitude of the momentum transfer $\Delta$, and of the recoil momentum of the ion $Q$, as a function of the scattering angle $\theta$, for a fully symmetric coplanar geometry, in the case of the laser-assisted (e, 2e) reaction, at an incident electron energy of $250 \mathrm{eV}$.

\begin{tabular}{ccccccc}
\hline $\boldsymbol{\theta}$ (deg) & $\begin{array}{c}\Delta \text { (a.u.) } \\
\boldsymbol{\ell = 0}\end{array}$ & $\begin{array}{c}\Delta \text { (a.u.) } \\
\boldsymbol{\ell = 1}\end{array}$ & $\begin{array}{c}\Delta \text { (a.u.) } \\
\boldsymbol{\ell = - 1}\end{array}$ & $\begin{array}{c}\mathbf{Q} \text { (a.u.) } \\
\boldsymbol{\ell = 0}\end{array}$ & $\begin{array}{c}\mathbf{Q} \text { (a.u.) } \\
\boldsymbol{\ell = 1}\end{array}$ & $\begin{array}{c}\boldsymbol{Q} \text { (a.u.) } \\
\boldsymbol{\ell}=-\mathbf{1}\end{array}$ \\
\hline 0 & 1.83427 & 1.82919 & 1.83936 & 2.39355 & 2.403718 & 2.38338 \\
10 & 2.03551 & 2.03116 & 2.03986 & 2.26509 & 2.27510 & 2.25507 \\
20 & 2.54084 & 2.53790 & 2.54378 & 1.88361 & 1.89316 & 1.87405 \\
30 & 3.19874 & 3.19712 & 3.20073 & 1.26071 & 1.26951 & 1.25189 \\
40 & 3.91878 & 3.91825 & 3.91933 & 0.41531 & 0.42309 & 0.40751 \\
50 & 4.65563 & 4.65599 & 4.65527 & 0.62191 & 0.62038 & 0.63345 \\
60 & 5.38461 & 5.38574 & 5.38348 & 1.83427 & 1.82919 & 1.83936 \\
70 & 6.09032 & 6.09211 & 6.08852 & 3.17009 & 3.16662 & 3.17357 \\
80 & 6.76184 & 6.76423 & 6.75946 & 4.59379 & 4.59202 & 4.59556 \\
90 & 7.39077 & 7.39368 & 7.38786 & 6.06210 & 6.06210 & 6.06210 \\
100 & 7.79023 & 7.97359 & 7.96686 & 6.53041 & 7.532172 & 7.52864 \\
130 & 9.35800 & 9.36241 & 9.35358 & 11.49729 & 11.50382 & 11.49075 \\
150 & 9.95063 & 9.95548 & 9.94579 & 13.38491 & 13.39371 & 13.37609 \\
180 & 10.28992 & 10.28992 & 10.28483 & 14.51775 & 14.51775 & 14.50750 \\
\hline
\end{tabular}


In Figure 23, we show the distribution of the multiphoton TDCS as a function of the number of exchanged photons $\ell$ for two cases where the laser polarization is either parallel or perpendicular to the incident momentum. The spectra are symmetric about $\ell=0$. The photon absorption processes $(\ell>0)$ dominate those of photon emission $(\ell<0)$; meaning that the system absorbs net energy from the radiation background. The cross sections for both geometries have the same shape, which is not the case in the coplanar asymmetric geometry. The TDCS for $\widehat{\varepsilon} / / \boldsymbol{k}_{i}$ (Figure 23b) present a cut-off (in $\ell= \pm 5$ ) which is due to the argument of the Bessel function $J_{\ell}$ in the scattering amplitude (Equation (60)), where $\Delta$ is very small (i.e., $\Delta . \alpha_{0}$ is as well very small) then the laser field plays a minor role and hence the weak influence of the laser field on the outcome of the collision process. This is because the energy absorbed by the electrons from the external laser field needs to be converted into a linear momentum via a re-scattering from the residual ion. It is to highlight that in these kinematical considerations, the overall magnitude of the TDCS is large for the laser polarization direction perpendicular to the incident momentum than for the polarization direction parallel to the wave vector of the incident electron.
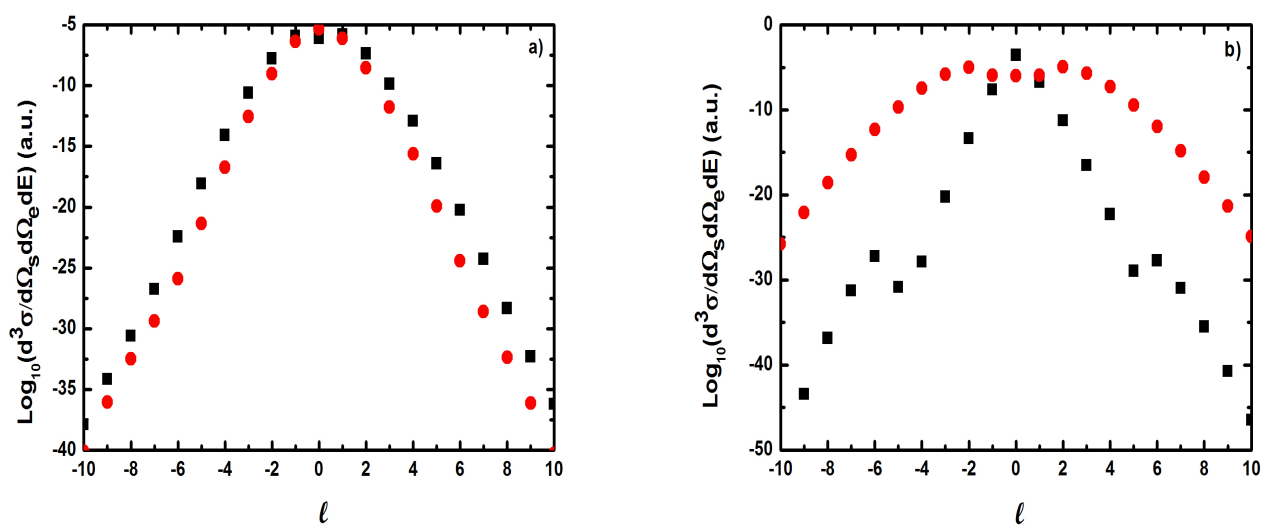

Figure 23. First Born triple differential cross sections corresponding to laser-assisted electron-impact ionization of atomic hydrogen in the ground state for the case of a coplanar symmetric geometry as a function of the number of photons exchanged $\ell$. The scattering angle is (a) $\theta=10^{\circ}$ and (b) $\theta=30^{\circ}$. The incident electron energy is $E_{k_{i}}=250 \mathrm{eV}$, the laser frequency is $\omega=1.17 \mathrm{eV}$ and the electric field strength is $\mathcal{E}_{0}=10^{7} \mathrm{~V} / \mathrm{cm}$. Squares: the laser polarization vector is taken to be parallel to the incident momentum. Circles: the laser polarization vector is taken to be perpendicular to the incident momentum.

In Figure 24, we show the variation of the TDCS for no photon transfer $\ell=0$ between the projectile-atom system and the laser field, i.e., the energies of the outgoing electrons are the same as those obtained in the field-free case. The influence of changing the laser field strength, everything else being kept fixed, on the angular distribution reflects the role of the dressing of the projectile-target system by the external beam without exchange of photons. The TDCS of laser-assisted electron-impact ionization deviates slightly from the field-free one for moderate laser intensities. The most remarkable differences are the following:

- For electric field strengths that are much smaller than the atomic unit $\left(\mathcal{E}_{0} \leq e / a_{0}^{2} \simeq 5 \times 10^{9} \mathrm{~V} / \mathrm{cm}\right)$ such that perturbation theory is applicable regarding the laser-atom interaction, the attraction between the electron and the nucleus becomes negligible and one can model the $(\mathrm{e}, 2 \mathrm{e})$ reaction as an electron-electron coupling. In other words, in the (e, 2e) spectroscopy region, the laser-assisted electron-impact ionization of atomic hydrogen in the coplanar symmetric geometry is governed by the electron-electron interaction and one can model the atom by a structureless center of force. Furthermore, for $\ell=0$, the dressing of the target states does not play a dominant role in the physics of the process. The first Born approximation to $(e, 2 e)$ scattering amplitude with the transfer of $\ell$ photons Equation (60) can be replaced by the expression (61). 
- The magnitude of the cross sections in the presence of the radiation field with no photon exchanged between the projectile-atom system and the laser field is comparable to that in the field-free case. This results from the fact that the laser itself does not contribute to the ionization process [88]. At the (e, 2e) spectroscopy region, the laser field redistributes the ejected electrons in new channels associated with indices $\ell \neq 0$ in the energy conservation relation, which are accessible in the "dressed" continuum of the atomic target. A simplified description of this process is that of two-step processes in the course of which the atomic electron is first brought into the continuum as a result of the collision, and then exchanges photons with the laser field [88].
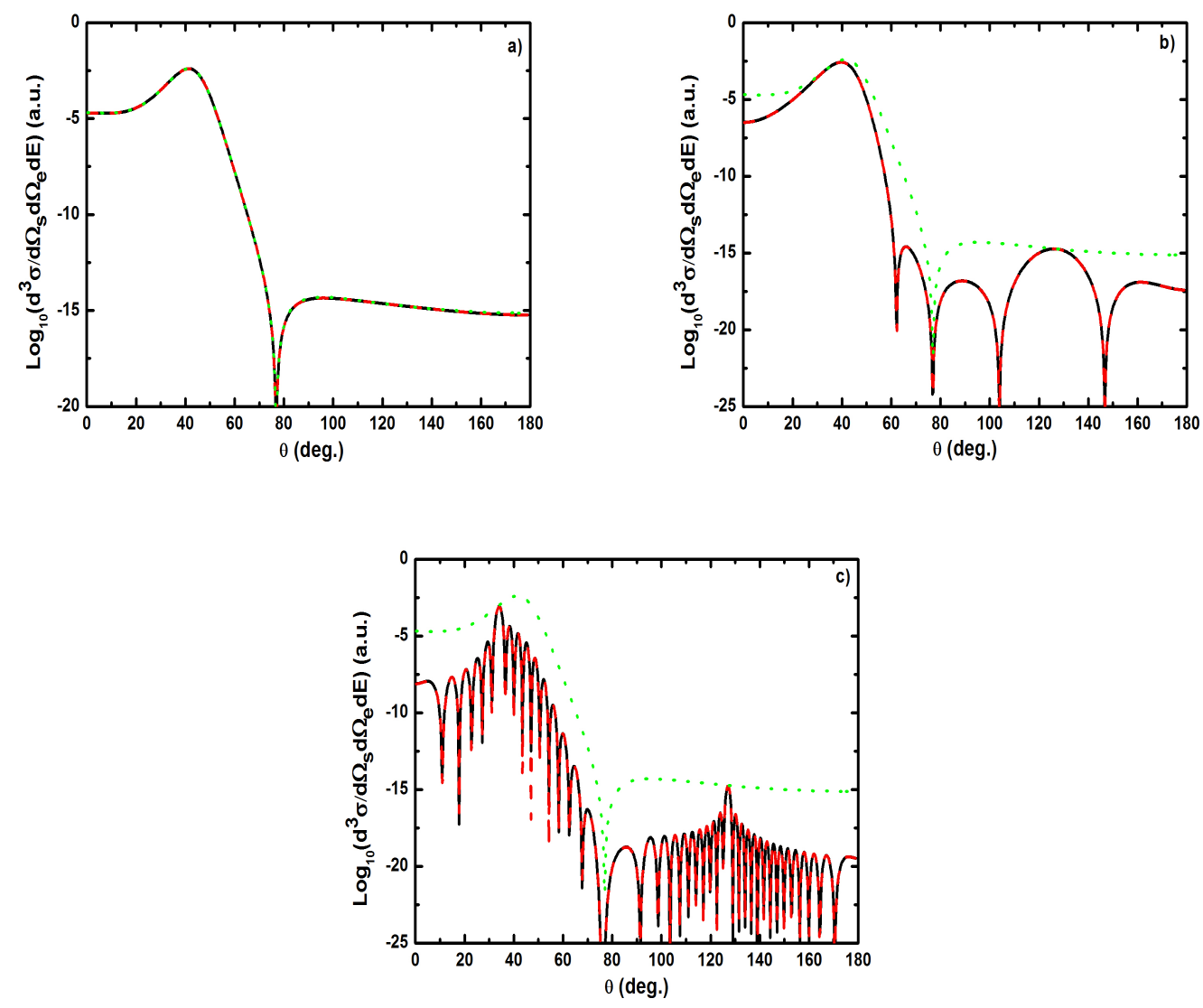

Figure 24. Triple differential cross sections corresponding to laser-assisted electron-impact ionization of atomic hydrogen in the ground state for the case of a coplanar symmetric geometry as a function of the scattering angle $\theta=\theta_{s}=\theta_{e}$ for no net exchange of photons $(\ell=0)$. The incident electron energy is $E_{k_{i}}=250 \mathrm{eV}$, the laser frequency is $\omega=1.17 \mathrm{eV}$ and the electric field strength is (a) $\mathcal{E}_{0}=10^{6} \mathrm{~V} / \mathrm{cm}$, (b) $\mathcal{E}_{0}=10^{7} \mathrm{~V} / \mathrm{cm}$ and (c) $\mathcal{E}_{0}=10^{8} \mathrm{~V} / \mathrm{cm}$. The laser polarization vector is taken to be parallel to the incident momentum $\boldsymbol{k}_{i}$. Solid lines: First Born approximation results. Dashed line: results obtained by neglecting the dressing of the target by the laser field. Dotted lines: field-free results.

We also notice significant changes in the overall magnitude of the cross section for symmetric (e, 2e) reactions in the range of large scattering angles (which corresponds to large momentum transfers). In the large angle region, the situation is more complicated for ionization processes, since in this case, the existence of the ejected electron momentum $\boldsymbol{k}_{e}$ gives much more kinematical possibilities than what is found in bound-bound processes. Indeed, we have already seen in the previous section that the recoil momentum $Q=\Delta-\boldsymbol{k}_{e}$, which can be constructed from the momentum transfer and the momentum of the ejected electron, is of great significance. As can be seen from Table 1, when the scattering angle becomes large both $\Delta$ and $Q$ become large in the symmetric energy-sharing geometry. The TDCS in the large $\Delta$ region is almost constant in the field-free case. An additional plateau structure appears after an 
abrupt cutoff. This phenomenon can be interpreted by the disappearance of the recoil peak which is governed by the attraction between the electron and the nucleus, i.e., the electron-nucleus interaction (term on $1 / \boldsymbol{r}_{01}$ ) at large angles. The physical interpretation of this phenomenon is that the plateau manifests itself as a broad energy interval of the spectrum, and the electron can absorb the energy from the laser field in the intermediate step of the ionization process and crosses the collision zone with the nucleus without interaction. We also observe oscillations in the TDCS when increasing the field strength intensity. It is clear that the Bessel function is responsible for such oscillations. This function is derived from the Volkov wave function, which reflects the effect of the laser field on the free electron. From a physical perspective, the oscillations may be linked to the oscillating component of the electron velocity, and hence of the energy of the electron during the scattering event. Another consequence of increasing the laser strength intensity for no net exchange of photons on the angular distribution of the outgoing electron is shown in Figure 24. In fact, the laser-assisted TDCS deviates very little from the field-free one, so long as the laser strength intensity is not larger than $10^{7} \mathrm{~V} / \mathrm{cm}$ (see Figure 24a,b). At higher intensities (Figure 24c), however, the angular distribution is strongly modified in (e, 2e) reaction. The magnitude of the cross section for no net exchange of photons is significantly smaller than the field-free case. For $\ell=0$ and at the relatively low laser intensity considered here, the dressing of the target states does not play a dominant role in the physics of the process.

We now turn to the discussion of the role of the external laser beam in the case of the net absorption of one laser photon $(\ell=1)$. As shown in Figure 25, the complete results obtained by using the ionization amplitude equation in the FBA are compared with those obtained by neglecting the dressing of the atomic target. We observe the emergence of small lobes with different magnitudes. Several interesting points, characteristic of the class of laser-assisted reactions, can be made at this stage:

- At low laser photon energies and low laser intensities considered here, the dressing of the target states does not play a dominant role in the physics of laser-assisted electron-impact ionization of atomic hydrogen in the coplanar symmetric energy sharing geometry. This result shows, in other words, that under the present conditions the term $f_{1}$ in Equation (61) is dominant in the (e, 2e) scattering amplitude Equation (60) and one can model the target by a structureless center of force. Note that the atom distortion by the external laser beam will be taken into account at high frequency field (see below). Such a distorted target also acts on the projectile by a long range dipole potential $\left(1 / \boldsymbol{r}^{2}\right)$, which requires a nonperturbative treatment of the laser-atom interaction at low incident electron energies.

- As shown in Table 1, when the incident electron is fast, the magnitude of the momentum transfer $K$ is never small in a symmetric geometry. On the other hand, the magnitude of the recoil momentum of the residual ion, can be either small, or it can become large. For scattering angles $\theta<70^{\circ}$, the values of $Q$ remain small ((e, 2e) spectroscopy region), while $Q$ becomes large for $\theta \geq 70^{\circ}$. In the (e, $\left.2 \mathrm{e}\right)$ spectroscopy region, the angular distribution illustrates that the electron-electron interaction which is attributed to the binary peak, is now split into two lobes with different magnitudes, while in large angles symmetric (e, 2e) reactions, the recoil peak (governed by the attraction between the electron and the nucleus) is split into smaller lobes with comparable magnitude. These lobes construct a plateau structures followed by an abrupt cutoff. We clearly observe an oscillatory behavior in the TDCS. This behavior can be traced back to the argument of the Bessel functions entering the expressions of the amplitude Equation (60) (because the dressing effect is less important in this case). For the net absorption of one photon $(\ell=1)$ the results are governed by binary collisions and the recoil collisions are suppressed significantly. We attribute them to the contribution of the intermediate states and the long-range dipole potential affects mainly the distant collisions, which contribute when the energies of the primary electron are weak and the second Born approximation is needed. Outside the (e, 2e) spectroscopy region, $Q$ becomes large and the simple first Born term of Equation (60) is no longer sufficient. Indeed, the second-order contribution of the ground intermediate state is of the same 
order of magnitude as the first Born term and therefore is essential to a complete description of the ionization amplitude.

- The triply differential cross sections displays two distinct minimums denoted $\left(m_{1}\right)$ and $\left(m_{2}\right)$. Both minima correspond to values of the scattering angles for which the cross section is actually zero, nevertheless the origin of these zeros is different for each case. The minimum denoted $m_{1}$ occurs in fact at angles $\theta_{m_{1}} \simeq 70^{\circ}$ such that the first Born amplitude for the laser-assisted (e, 2e) process involving the exchange of $\ell$ photons vanishes. At the relatively low laser intensity considered here, the dressing of the target states does not play a dominant role and under the present conditions the term $f_{1}$ is dominant and that is zero at $\theta_{m_{1}}$ scattering angle. In fact, it is the angle that separates the (e, 2e) spectroscopy region (for small scattering angles $\theta_{m_{1}}<70^{\circ}$, the values of $Q$ remain small while in the large angle symmetric (e, 2e) region $Q$ becomes large). On the other hand, the other minimums $m_{2}$ are due to the argument of the Bessel functions entering the expressions of the amplitudes Equation (60), which grows with $\mathcal{E}_{0}$ and varies with the scattering angle. At higher field strengths, every other laser parameters being fixed, one explores more zeros of the Bessel functions when varying the scattering angle. This clearly gives rise to the observed increase in the number of minima. The same phenomena occurs for the case where the laser polarization vector is perpendicular to the incident momentum (not presented in the present paper).
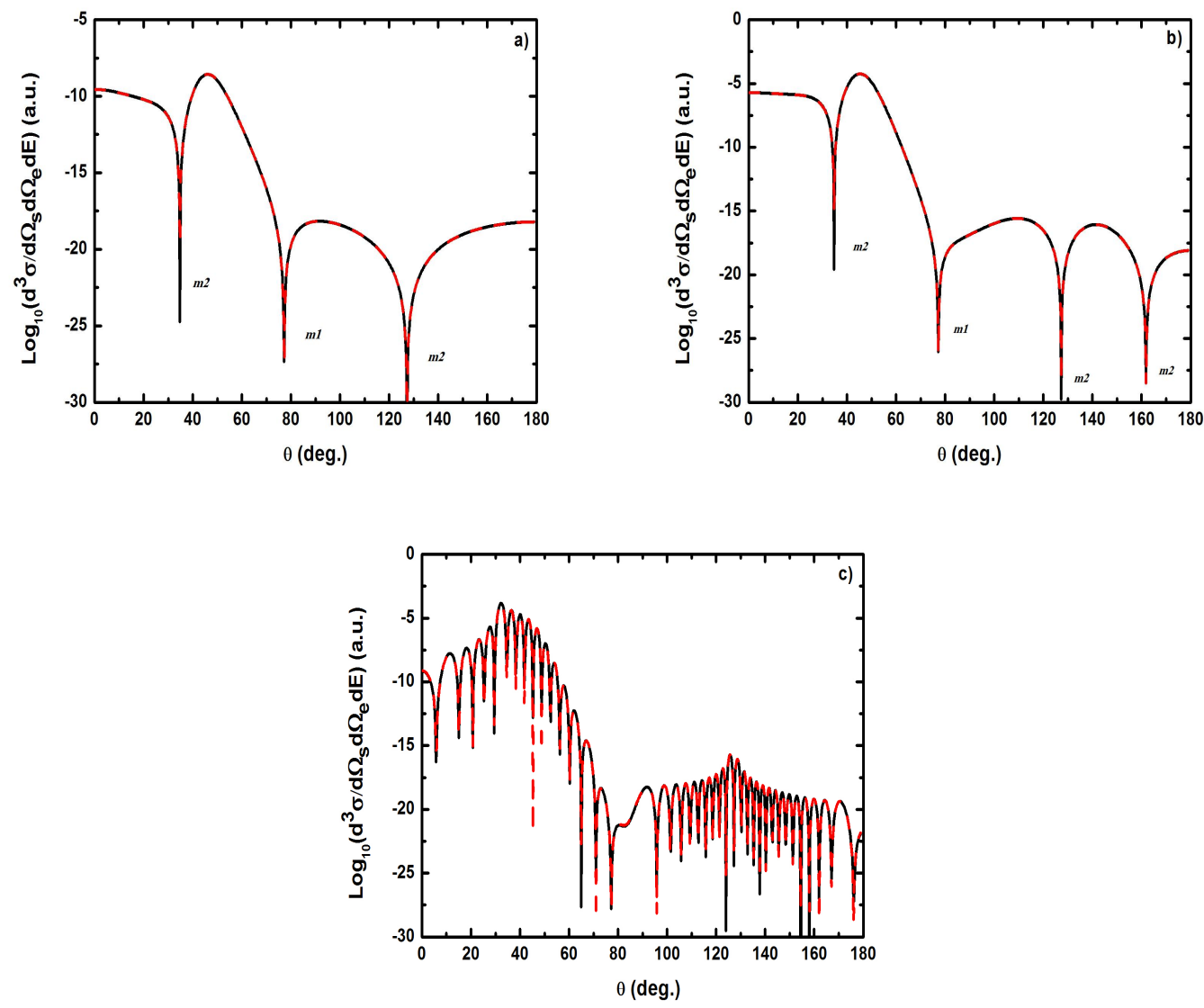

Figure 25. All the parameters are the same as Figure 24, but with the absorption of one photon $(\ell=1)$.

We are now interested in demonstrating the effects of the laser frequency on the laser-assisted electron-impact ionization of hydrogen target in the symmetric coplanar geometry. The laser polarization vector is taken to be parallel or perpendicular to the incident momentum. In Figures 26 and 27, we show the variation of the TDCS when the ionizing process is accompanied by the absorption of one photon $(\ell=1)$ for different values of the laser photon energy and the field 
strength is kept fixed at $1 \times 10^{6} \mathrm{~V} \cdot \mathrm{cm}^{-1}$. We present the results of our complete computation of TDCS in the FBA and compare them with those obtained by neglecting the target dressing effects. We have chosen 4 laser frequencies which are typical of laser sources currently operated, namely $\omega=1.17 \mathrm{eV}$ (Nd-YAG laser:yttrium aluminum garnet, Figure 25a), $4.95 \mathrm{eV}\left(\mathrm{Kr}^{*} \mathrm{~F}\right.$ laser), $6.42 \mathrm{eV}$ (Ar*F laser), and $11.7 \mathrm{eV}$ (tenth harmonic of Nd:YAG laser). It is seen that dressing effects of the target are somewhat insignificant at low frequencies, while at relatively high laser frequencies, the dressing of the of the atomic target by the laser field can significantly affect the cross sections corresponding to the ionizing process with the net exchange of one photon $(\ell=1)$, a result which implies that the contribution of the atomic amplitudes $f_{2}$ and $f_{3}$ becomes dominant. This clearly shows the inadequacy of the simplified approach in which one neglects the dressing of the atomic target states by the field at high laser frequencies. The most remarkable feature of the frequency dependence of the TDCS is the fact that the magnitude of the cross section decreases when the laser frequency increases. Another consequence of increasing the laser photon energy is that the electron-electron interaction becomes more important than the electron-nucleus attraction. At higher laser frequencies (see Figures 26c and 27c), every other laser parameter being fixed, one explores more zeros of the angular distribution. These oscillations reflect the highly non-perturbative nature of the interaction of the laser field with the free electron.
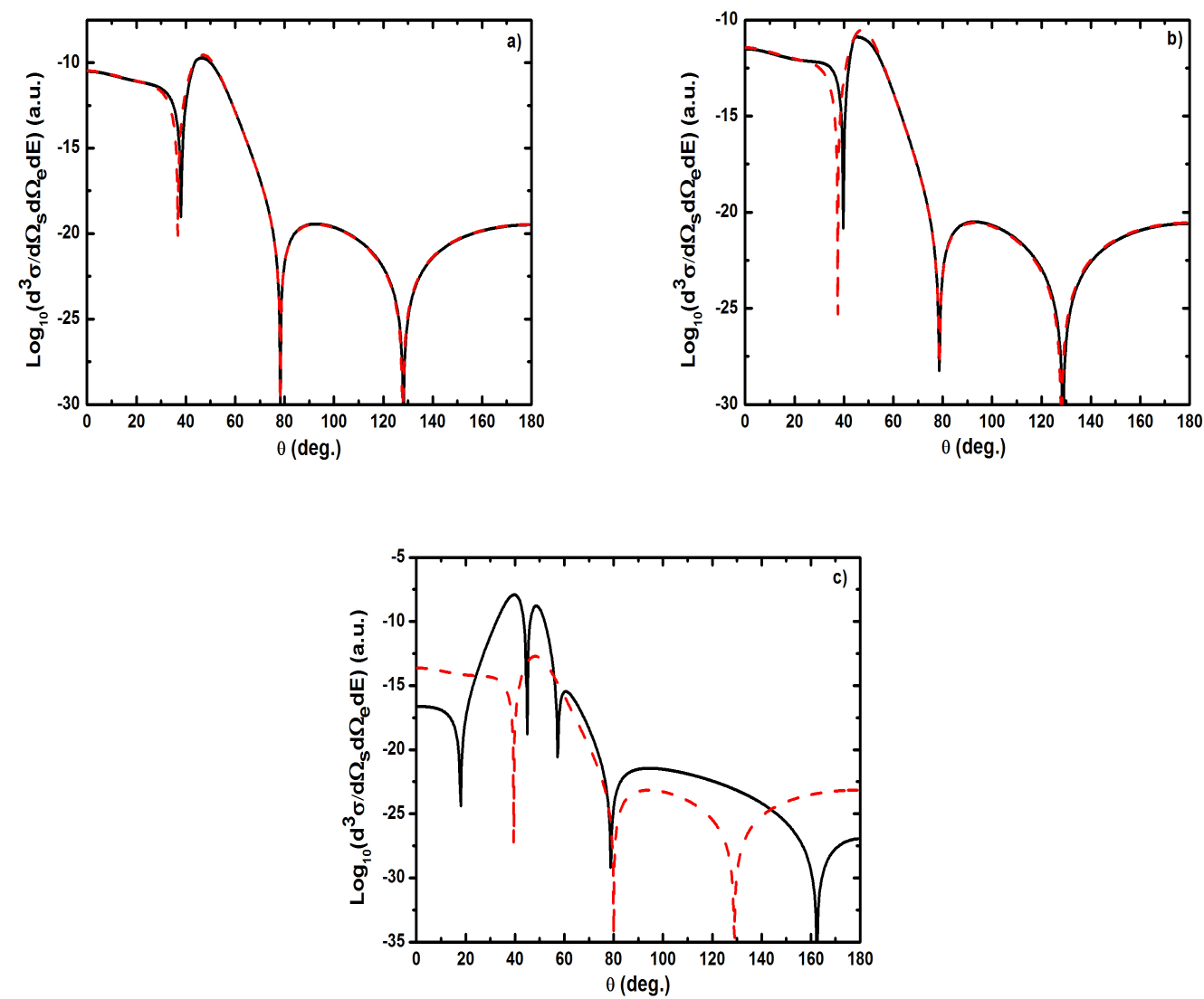

Figure 26. Triple differential cross sections corresponding to laser-assisted electron-impact ionization of atomic hydrogen in the ground state for the case of a coplanar symmetric geometry as a function of scattering angle $\theta=\theta_{s}=\theta_{e}$ for the absorption of one photon $(\ell=1)$. The incident electron energy is $E_{k_{i}}=250 \mathrm{eV}$, and the electric field strength is kept fixed at $\mathcal{E}_{0}=10^{6} \mathrm{~V} / \mathrm{cm}$. The laser photon energy is (a) $\omega=4.95 \mathrm{eV}$; (b) $\omega=6.42 \mathrm{eV}$; (c) $\omega=11.7 \mathrm{eV}$. The laser polarization vector is taken to be parallel to the incident momentum $\boldsymbol{k}_{i}$. Solid lines: First Born approximation results. Dashed line: results obtained by neglecting the dressing of the target by the laser field. 

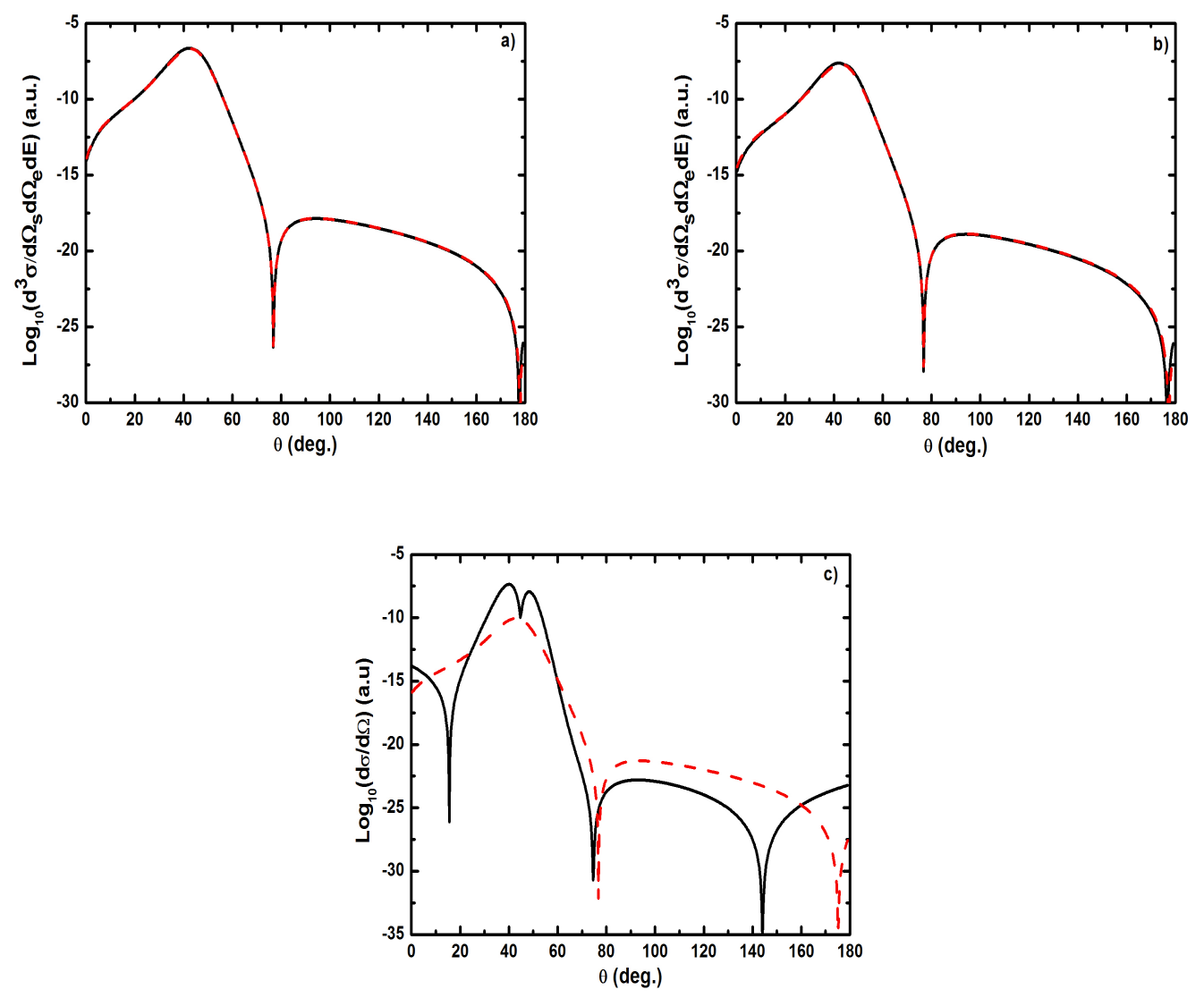

Figure 27. All the parameters are the same as Figure 26, but the laser polarization vector is taken to be perpendicular to the incident momentum $\boldsymbol{k}_{i}$.

\section{Conclusions}

In this review paper, we have presented a theoretical treatment and analysis of laser-assisted triple differential cross sections corresponding to electron-impact ionization $(\mathrm{e}, 2 \mathrm{e})$ collisions in atomic hydrogen. Two kinematical arrangements are chosen: the coplanar symmetric and the coplanar asymmetric geometries. Our treatment consistently includes the dressing of the atomic target by the laser field. In addition, the exchange effects have been considered. Particular attention has been paid to the construction of dressed laser-modified initial and final atomic wave functions. For the coplanar asymmetric geometry, dramatic changes in the triply differential cross sections can occur when varying the laser parameters: field strength, frequency, and polarization orientation. Further, it has been shown that when varying the incident electron energy, the angular distribution of the ejected electron can be strongly modified. Comparing with the standard first-order Born calculations, the inclusion of the second-order Born term in the ionizing amplitude does not lead to any change in the TDCS at large incident electron energies, whereas in the low energy range the present second-order corrections are seen to be important. The second-order corrections are significant at low incoming energies for no net exchange of photons and for the stimulated emission of photons, while they are weak when the ionizing process is accompanied by the absorption of photons. The inclusion of the second-order Born term improves TDCS calculations in the low incident energy region especially in the vicinity of the recoil peaks. The influence of the laser polarization orientation plays an obvious role in the TDCS for high energy electron collision, but is insensitive in the low energy range. In the symmetric energy sharing kinematics, the importance of dressing effects and the influence of laser parameters on the TDCS has been discussed, as well as the structure of the angular distribution of the electron-electron and electron-nucleus interaction in $(\mathrm{e}, 2 \mathrm{e})$ spectroscopy region. We have clearly demonstrated the 
effects of the laser field strength in laser-assisted (e, 2e) collisions. We have also illustrated the influence of the laser frequency by comparing the triple differential cross sections for suitable choices of laser photon energies. Outside the (e, 2e) spectroscopy region, the second-order Born contribution is of paramount importance and would be significant. Up to now, no laser-assisted (e, 2e) experiment on atomic hydrogen is yet available in the literature. The absence of any experimental data adds further importance to the theoretical study of such a process. We believe that our results should serve as an incentive to perform such laser-assisted collision experiments.

Author Contributions: Conceptuation, A.M. and I.A.; Methodology, A.M.; Software, D.K.; Validation, D.K. and I.A.; Formal analysis, A.M.; Investigation, A.M. and D.K.; Resources, A.M.; Data curation, A.M. and D.K.; Writing-original draft preparation, A.M. and I.A.; Writing—review and editing, A.M. and I.A.; Visilization, A.M.; Supervision, A.M.; Project administration, A.M., Funding acquisition, A.M.

Funding: This research received no external funding.

Conflicts of Interest: The authors declare no conflict of interest.

\section{Appendix A. Sturmian Expansion of the First-Order Perturbed Wavefunction}

We shall consider the computation of second-order perturbative radial amplitudes of the general form

$$
M=<R\left|V_{1}(r) G_{\lambda}(\Omega) V_{2}(r)\right| R^{\prime}>,
$$

where $V_{1}$ and $V_{2}$ are any radial perturbation operators, and the kets $\mid R>$ and $\mid R^{\prime}>$ represent Coulomb radial functions, which can belong to either the discrete or continuous spectrum.

The computation of the radial amplitudes $M$, can present some severe difficulties, especially when the argument $\Omega$ of the Coulomb Green's function is positive. In order to overcome this difficulty, we have decided to use a powerful technique based on the use of a Coulomb Sturmian basis. The basic idea lies in the expansion onto the radial Sturmian basis, of the perturbed wave functions $\left|\psi^{(1)}\right\rangle$.

The amplitude $M$ can be rewritten as

$$
M=<R\left|V_{1}(r)\right| \psi^{(1)}>.
$$

where $\mid \psi^{(1)}>$ is the first-order perturbed radial function, with angular momentum $\lambda$, corresponding to the state $\mid n, \ell>$ perturbed by the potential $V_{2}$

$$
\left|\psi^{(1)}>=G_{\lambda}(\Omega) V_{2}(r)\right| R^{\prime}>,
$$

which is the solution of the following inhomogeneous differential equation

$$
\left(\Omega-H_{\lambda}+\frac{1}{r}\right)\left|\psi^{(1)}>=V_{2}(r)\right| R^{\prime}>,
$$

$\left|\psi^{(1)}\right\rangle$ can be expanded onto the Sturmian basis

$$
\left|\psi^{(1)}\right\rangle=\sum_{n} c_{n}\left|S_{n}\right\rangle
$$

The vector $C$, with components $c_{n}$ is solution of the matrix equation

$$
A \cdot C=B
$$

where $A$, which represents the Hamiltonian operator $\left(\Omega-H_{\lambda}+\frac{1}{r}\right)$, turns out to be a tridiagonal matrix in the Sturmian'basis. The fact that $A$ is tridiagonal results from the orthogonality and scalar product properties of the Sturmians. One has, more specifically 


$$
\left(\begin{array}{cccccc}
\alpha_{1} & \beta_{1} & 0 & 0 & \ldots & 0 \\
\gamma_{2} & \alpha_{2} & \beta_{2} & 0 & \ldots & 0 \\
0 & \gamma_{3} & \alpha_{3} & \beta_{3} & \ldots & 0 \\
0 & 0 & \gamma_{4} & \alpha_{4} & \ldots & 0 \\
\ldots & \ldots & \ldots & \ldots & \ldots & \beta_{n-1} \\
0 & 0 & 0 & 0 & \gamma_{n} & \alpha_{n}
\end{array}\right) \times\left(\begin{array}{c}
c_{1} \\
c_{2} \\
c_{3} \\
c_{4} \\
\ldots \\
c_{n}
\end{array}\right)=\left(\begin{array}{c}
b_{1} \\
b_{2} \\
b_{3} \\
b_{4} \\
\ldots \\
b_{n}
\end{array}\right)
$$

where $\alpha_{n}, \beta_{n}, \gamma_{n}$ are defined by the expressions

$$
\begin{gathered}
\alpha_{n}=1-n x+\left(\Omega+\frac{x^{2}}{2}\right) \frac{n}{x}, \\
\beta_{n}=-\left(\Omega+\frac{x^{2}}{2}\right) \frac{1}{2 x} \sqrt{(n+\ell+1)(n-\ell)}, \\
\gamma_{n}=-\left(\Omega+\frac{x^{2}}{2}\right) \frac{1}{2 x} \sqrt{(n+\ell)(n-\ell-l)},
\end{gathered}
$$

On the other hand, the components of the vector $B$ are of the general form

$$
b_{n}=<S_{n}\left|V_{2}(r)\right| R^{\prime}>.
$$

which, in most applications can he expressed in closed form in terms of hypergeometric polynomials. The components of the vector $C$ are then straightforwardly obtained by solving the tridiagonal system, Equation (A7), and the required transition amplitude $M$ is given by the infinite sum

$$
M=\sum_{n} c_{n}<R\left|V_{1}(r)\right| S_{n}>.
$$

The radial Coulomb Sturmian functions for angular momentum $\ell$ are solutions of the radial equation

$$
-\frac{1}{2}\left[\frac{d^{2}}{d r^{2}}+\frac{2}{r} \frac{d}{d r}+2 E+\frac{\ell(\ell+1)}{r^{2}}\right] S_{n \ell E}(r)=-\alpha_{n \ell E} V(r) S_{n \ell E}(r) .
$$

where

$$
\alpha_{n \ell E}=n x ; \quad x=\sqrt{-2 E} ; \quad n=\ell+1, \ell+2, \ldots
$$

One has also

$$
S_{n \ell E}(r)=N_{n, \ell} e^{-x r} r^{\ell}{ }_{1} F_{1}(\ell+1-n, 2 \ell+2,2 x r),
$$

with

$$
N_{n, \ell}=\frac{(2 x)^{\ell+1}}{(2 \ell+1) !} \sqrt{\frac{(n+\ell) !}{(n-\ell-1) !}} .
$$

\section{Appendix B. Calculation of the Exact Upper Boundary of the Integral Over the Virtual Projectile}

The integral of Equation (40) over the virtual projectile states $\chi_{q}\left(\boldsymbol{r}_{0}, t\right)$ with wavevector $\boldsymbol{q}$ is prohibitively difficult, which is actually zero at some values of incident electron energy and accordingly for some values of scattered electron energy. Each of these possible intermediate transitions will be characterized by a resonance behavior, i.e., the denominator of the matrix element entering the exact formula Equation (38) being close to zero. Instead, we shall overcome this difficulty by determining the exact upper boundary of the integral Equation (40) over the virtual projectile, which can be derived as follows.

Starting from the definitions used

$$
\Delta_{f}=q-k_{f}
$$




$$
\Delta_{f} \cdot \boldsymbol{q}=\Delta_{f} q \cos \left(\widehat{\Delta_{f}, \boldsymbol{q}}\right)
$$

and

$$
\Delta_{f} \cdot \boldsymbol{q}=\left(\boldsymbol{q}-\boldsymbol{k}_{f}\right) \cdot \boldsymbol{q}=q^{2}-q k_{f} \cos \left({\widehat{\boldsymbol{q}}, \boldsymbol{k}_{f}}\right)
$$

we find that

$$
\Delta_{f}^{2} \cos ^{2}\left(\widehat{\Delta_{f}, \boldsymbol{q}}\right)=q^{2}+k_{f}^{2} \cos ^{2}\left(\widehat{\boldsymbol{q}, \boldsymbol{k}_{f}}\right)-2 q k_{f} \cos \left(\widehat{\boldsymbol{q}, \boldsymbol{k}_{f}}\right) .
$$

we obtain a second-order equation on $\cos (\widehat{\boldsymbol{q}, \boldsymbol{k}})$, whose acceptable solution, namely

$$
k_{f}^{2}-q^{2} \sin ^{2}\left({\widehat{\boldsymbol{q}}, \Delta_{f}}\right) \geq 0 .
$$

then

$$
q \leq k_{f}
$$

In the same way and by using the relation $\Delta_{i}=\boldsymbol{k}_{i}-\boldsymbol{q}$, we obtain the following inequality

$$
q \leq k_{i}
$$

Finally, by combining the inequalities Equations (A22) and (A23), we deduce that the upper boundary of the integral over the virtual projectile is

$$
q \leq \inf \left(k_{i}, k_{f}\right) .
$$

\section{References}

1. Brantov, A.; Rozmus, W.; Sydora, R. Enhanced inverse bremsstrahlung heating rates in a strong laser field. Phys. Plasmas 2003, 10, 3385-3396. [CrossRef]

2. Wang, F.; Weckert, E.; Ziaja, B. Inverse bremsstrahlung cross section estimated within evolving plasmas using effective ion potentials. J. Plasma Phys. 2009, 75, 289-301. [CrossRef]

3. Wallbank, B.; Holmes, J.K. Differential cross sections for laser-assisted elastic electron scattering from argon. J. Phys. B 1994, 27, 5405. [CrossRef]

4. Wallbank, B.; Holmes, J.K. Low-energy electron-helium scattering in a laser field. J. Phys. B 1996, $29,5881$. [CrossRef]

5. Wallbank, B.; Holmes, J.K. Laser-assisted elastic electron scattering from helium. Can. J. Phys. 2001, 79, 1237-1246. [CrossRef]

6. Nehari, D.; Holmes, J.; Dunseath, K.M.; Terao-Dunseath, M. Experimental and theoretical study of free-free electron-helium scattering in a $\mathrm{CO}_{2}$ laser field. J. Phys. B 2010, 43, 025203. [CrossRef]

7. DeHarak, B.A.; Ladino, L.; MacAdam, K.B.; Martin, N.L.S. High-energy electron-helium scattering in a Nd: YAG laser field. Phys. Rev. A 2011, 83, 022706. [CrossRef]

8. Musa, M.O.; MacDonald, A.; Tidswell, L.; Holmes, J.; Wallbank, B. Laser-induced free-free transitions in elastic electron scattering from $\mathrm{CO}_{2}$. J. Phys. B 2010, 43, 175201. [CrossRef]

9. Mason, N.J.; Newell, W.R. The polarisation dependence of the simultaneous electron-photon excitation cross section of the helium $2^{3} S$ state. J. Phys. B 1990, 23, L179. [CrossRef]

10. Wallbank, B.; Holmes, J.K.; Weingartshofer, A. Absorption and emission of radiation during electron excitation of the $21 \mathrm{~S}$ and $21 \mathrm{P}$ states of helium. Phys. Rev. A 1989, 40, 5461. [CrossRef]

11. Wallbank, B.; Holmes, J.K.; Weingartshofer, A. Simultaneous electron-photon excitation of He $2^{3} S$ : An experimental investigation of the effects of laser intensity and polarisation. J. Phys. B 1990, 23, 2997. [CrossRef]

12. Luan, S.; Hippler, R.; Lutz, H.O. Simultaneous electron-photon excitation of helium (h(cross) omega $=1.17 \mathrm{eV}$ ). J. Phys. B 1991, 24, 3241. [CrossRef]

13. Ehrhardt, H.; Schulz, M.; Tekaat, T.; Willmann, K. Ionization of helium: Angular correlation of the scattered and ejected electrons. Phys. Rev. Lett. 1969, 22, 89. [CrossRef]

14. Röder, J.; Ehrhardt, H.; Pan, C.; Starace, A.F.; Bray, I.; Fursa, D.V. Absolute Triply Differential (e, 2e) Cross Section Measurements for H with Comparison to Theory. Phys. Rev. Lett. 1997, 79, 1666. [CrossRef] 
15. Röder, J.; Baertschy, M.; Bray, I. Measurements of the ionization of atomic hydrogen by 17.6-eV electrons. Phys. Rev. A 2003, 67, 010702. [CrossRef]

16. Childers, J.G.; James, K.E.; Hughes, M.; Bray, I.; Baertschy, M.; Khakoo, M.A. Electron-impact ionization of atomic hydrogen at incident electron energies of 15.6, 17.6, 25, and $40 \mathrm{eV}$. Phys. Rev. A 2003, 68, 030702. [CrossRef]

17. Dürr, M.; Dimopoulou, C.; Dorn, A.; Najjari, B.; Bray, I.; Fursa, D.V.; Chen, Z.; Madison, D.H.; Bartschat, K.; Ullrich, J. Single ionization of helium by $102 \mathrm{eV}$ electron impact: three-dimensional images for electron emission. J. Phys. B 2006, 39, 4097. [CrossRef]

18. Ren, X.; Senftleben, A.; Pflüger, T.; Dorn, A.; Colgan, J.; Pindzola, M.S.; Al-Hagan, O.; Madison, D.H.; Bray, I.; Fursa, D.V.; et al. Tracing multiple scattering patterns in absolute (e, 2e) cross sections for $\mathrm{H}_{2}$ and He over a $4 \pi$ solid angle. Phys. Rev. A 2010, 82, 032712. [CrossRef]

19. Ren, X.; Bray, I.; Fursa, D.V.; Colgan, J.; Pindzola, M.S.; Pflüger, T.; Senftleben, A.; Xu, S.; Dorn, A.; Ullrich, J. Phys. Rev. A 2011, 83, 052711. [CrossRef]

20. Höhr, C.; Dorn, A.; Najjari, B.; Fischer, D.; Schröter, C.D.; Ullrich, J. Electron Impact Ionization in the Presence of a Laser Field: A Kinematically Complete Experiment. (n $\gamma, 2 \mathrm{e})$. Phys. Rev. Lett. 2005, 94, 153201.

21. Höhr, C.; Dorn, A.; Najjari, B.; Fischer, D.; Schröter, C.D.; Ullrich, J. Laser-assisted electron-impact ionization of atoms. J. Electron Spectrosc. Relat. Phenom. 2007, 161, 172-177. [CrossRef]

22. Mohan, M.; Chand, P. Electron-impact ionization of the hydrogen atom in the presence of an intense laser beam. Phys. Lett. A 1978, 65, 399-401. [CrossRef]

23. Cavaliere, P.; Ferrante, G.; Leone, C. Particle-atom ionising collisions in the presence of a laser radiation field. J. Phys. B 1980, 13, 4495. [CrossRef]

24. Banerji, J.; Mittleman, M.H. Electron-atom ionising collisions in the presence of a low-frequency laser field. J. Phys. B 1981, 14, 3717. [CrossRef]

25. Cavaliere, P.; Leone, C.; Zangara, R.; Ferrante, G. Effects of a laser field on electron-atom ionizing collisions. Phys. Rev. A 1981, 24, 910. [CrossRef]

26. Zangara, R.; Cavaliere, P.; Leone, C.; Ferrante, G. Influence of laser properties on particle-atom ionising collisions. J. Phys. B 1982, 15, 3881. [CrossRef]

27. Zarcone, M.; Moores, D.L.; McDowell, M.R.C. Laser-assisted electron impact ionisation of helium at $256.5 \mathrm{ev.}$ J. Phys. B 1983, 16, L11. [CrossRef]

28. Mandal, C.K.M.; Ghosh, A.S. Electron-hydrogen ionization in the presence of a laser field. Phys. Rev. A 1984, 30, 2759. [CrossRef]

29. Joachain, C.J.; Francken, P.; Maquet, A.; Martin, P.; Véniard, V. (e, 2e) Collisions in the Presence of a Laser Field. Phys. Rev. Lett. 1988, 61, 165. [CrossRef]

30. Martin, P.; Véniard, V.; Maquet, A.; Francken, P.; Joachain, C.J. Electron-impact ionization of atomic hydrogen in the presence of a laser field. Phys. Rev. A 1989, 39, 6178. [CrossRef]

31. Khalil, D.; Maquet, A.; Taïeb, R.; Joachain, C.J.; Makhoute, A. Laser-assisted collisions in helium. Phys. Rev. A 1997, 56, 4918. [CrossRef]

32. Makhoute, A.; Khalil, D.; Maquet, A.; Taïeb, R. Light polarization effects in laser-assisted (e, 2e) collisions in helium. J. Phys. B 1999, 32, 3255. [CrossRef]

33. Jones, S.; Madison, D.H. Slow convergence of the Born approximation for electron-atom ionization. Phys. Rev. A 2002, 66, 062711. [CrossRef]

34. Li, S.M.; Chenand, J.; Zhou, Z.F. Electron-atomic-hydrogen "elastic" scattering in the presence of a laser field. Eur. Phys. J. D 2002, 19, 157. [CrossRef]

35. Li, S.M.; Chen, J.; Zhou, Z.F. Ionization of atomic hydrogen by protons in the presence of a laser field. J. Phys. B 2002, 35, 557. [CrossRef]

36. Duchateau, G.; Cormier, E.; Gayet, R. Coulomb-Volkov approach of ionization by extreme-ultraviolet laser pulses in the subfemtosecond regime. Phys. Rev. A 2002, 66, 023412. [CrossRef]

37. Colgan, J.; Pindzola, M.S.; Childers, G.; Khakoo, M.A. Low-energy electron-impact single ionization of helium. Phys. Rev. A 2006, 73, 042710. [CrossRef]

38. Stelbovics, A.T.; Bray, I.; Fursa, D.V.; Bartschat, K. Electron-impact ionization of helium for equal-energy-sharing kinematics. Phys. Rev. A 2005, 71, 052716. [CrossRef]

39. Wang, Y.; Jiao, L.; Zhou, Y. Second-order Born effect in coplanar doubly symmetric (e, 2e) collisions for sodium. Phys. Lett. A 2012, 376, 2122. [CrossRef] 
40. Dal Cappello, C.; Haddadou, A.; Menas, F.; Roy, A.C. The second Born approximation for the single and double ionization of atoms by electrons and positrons. J. Phys. B 2011, 44, 015204. [CrossRef]

41. Ajana, I.; Makhoute, A.; Khalil, D.; Dubois, A. The second Born approximation in laser-assisted (e, 2e) collisions in hydrogen. J. Phys. B 2014, 47, 175001. [CrossRef]

42. Makhoute, A.; Ajana, I.; Khalil, D. Low-energy electron-impact laser-assisted ionization of atomic hydrogen. Phys. Rev. A 2014, 90, 053415. [CrossRef]

43. Ajana, I.; Makhoute, A.; Khalil, D.; Chaddou, S. Exchange effects and second-order Born corrections in laser-assisted collisions with helium atoms. Phys. Rev. A 2015, 91, 043411. [CrossRef]

44. Makhoute, A.; Ajana, I.; Khalil, D.; Chaddou, S. Second-order Born calculation of laser-assisted single ionization of helium by electrons. Eur. Phys. J. D 2015, 69, 160. [CrossRef]

45. Ajana, I.; Makhoute, A.; Khalil, D. Laser polarization orientations in (e, 2e) reactions in atoms. Chin. Phys. Lett. 2015, 32, 8083401. [CrossRef]

46. Makhoute, A.; Ajana, I.; Khalil, D.; Taoutioui, A.; Dubois, A. Signatures of laser photon energy in (e, 2e) reactions in helium. Eur. Phys. J. D 2016, 70, 249. [CrossRef]

47. Khalil, D.; Tlidi, M.; Makhoute, A.; Ajana, I. Laser-assisted coplanar symmetric (e, 2e) triple differential cross sections. J. Phys. B 2017, 50, 078001. [CrossRef]

48. Ajana, I.; Agueny, H.; Khalil, D.; Makhoute, A. Laser-assisted (e, 2e) collisions in the coplanar symmetric geometry. J. Phys. B 2018, 51, 225005. [CrossRef]

49. Li, S.M.; Berakder, J.; Zhang, S.T.; Chen, J. Ionization of one-electron atoms and ions upon charged-particle impact assisted by a laser field. J. Electron. Spectrosc. 2007, 161, 188. [CrossRef]

50. Ghosh Deb, S.; Roy, S.; Sinha, C. Multiphoton (e, 2e) process of hydrogen atom in strong laser field. Eur. Phys. J. D 2009, 55, 591. [CrossRef]

51. Kouzakov, K.A.; Yu, V.; Popov, M.; Takahashi, A. Laser-assisted electron momentum spectroscopy. Phys. Rev. A 2010, 82, 023410. [CrossRef]

52. Bulychev, A.A.; Kouzakov, K.A.; Popov, Y.V. The role of Volkov waves in laser-assisted electron momentum spectroscopy. Phys. Lett. A 2012, 376, 484-487. [CrossRef]

53. Nixon, K.L.; Murray, A.J. Parametrization of Electron-Impact Ionization Cross Sections from Laser-Excited and Aligned Atoms. Phys. Rev. Lett. 2014, 112, 023202. [CrossRef]

54. Yamazaki, M.; Oishi, K.; Nakazawa, H.; Zhu, C.; Takahashi, M. Molecular Orbital Imaging of the Acetone $S_{2}$ Excited State Using Time-Resolved (e, 2e) Electron Momentum Spectroscopy. Phys. Rev. Lett 2015, 114, 103005. [CrossRef] [PubMed]

55. Tang, Z.; Shan, X.; Liu, Z.; Niu, S.; Wang, E.; Chen, X. Development of an electron momentum spectrometer for time-resolved experiments employing nanosecond pulsed electron beam. Rev. Sci. Instrum. 2018, 89, 033101. [CrossRef]

56. Zheng, M.Y.; Li, S.M. Laser-assisted ionization of atomic hydrogen by electrons in the second Born approximation. Phys. Rev. A 2010, 82, 023414. [CrossRef]

57. Volkov, D.M. On a class of solutions of the Dirac equation. Z. Phys. 1935, 94, 250-260.

58. Ehrhardt, H.; Hesselbacher, K.H.; Jung, K.; Schulz, M.; Willmann, K. Collisional ionization of helium by $250 \mathrm{eV}$ electrons: Experiments with completely determined kinetics. J. Phys. B 1972, 5, 2107. [CrossRef]

59. Beaty, E.C.; Hesselbacher, K.H.; Hong, S.P.; Moore, I.H. Triple-differential three-dimensional cross sections for low-energy electron impact ionization of helium. J. Phys. B 1977, 10, 611. [CrossRef]

60. Ehrhardt, H.; Fischer, M.; Jung, K. Triple differential cross section measurements for the electron impact ionization of helium: Comparison with a second Born approximation. Z. Phys. A 1982, 304, 119-124. [CrossRef]

61. Ehrhardt, H.; Fischer, M.; Jung, K.; Byron, F.W., Jr.; Joachain, C.; Piraux, B. Triply differential cross sections for the ionization of helium by fast electrons. Phys. Rev. Lett. 1982, 48, 1807. [CrossRef]

62. Lahmam-Bennani, A.; Wellenstein, H.F.; Duguet, A.; Rouault, M. Absolute triple differential cross sections for the $3 p$ ionisation of argon by electron impact. J. Phys. B 1983, 16, 121. [CrossRef]

63. Lahmam-Bennani, A.; Wellenstein, H.F.; Duguet, A.; Dal Cappello, C. Coincidence electron impact ionisation of helium: Absolute experimental cross sections and comparison with first-order theories. J. Phys. B 1984, 17, 3159. [CrossRef]

64. Jung, K.; Müller-Fiedler, R.; Schlemmer, P.; Ehrhardt, H.; Kiar, H. Absolute triple differential cross sections of electron impact ionisation of helium at $600 \mathrm{eV}$ collision energy. J. Phys. B 1985, 18, 2955. [CrossRef] 
65. Lahmam-Bennani, A.; Duguet, A.; Dal Cappello, C. High momentum range of electron momentum distributions investigated by means of high energy (e, 2e) collisions. J. Electron Spectrosc. 1986, 40, 141-161. [CrossRef]

66. Ehrhardt, H.; Jung, K.; Knoth, G.; Schlemmer, P. Differential cross sections of direct single electron impact ionization. Z. Phys. D 1986, 1, 3-32. [CrossRef]

67. Amaldi, U., Jr.; Egidi, A.; Marconero, R.; Pizzella, G. Use of a two channeltron coincidence in a new line of research in atomic physics. Rev. Sci. Instr. 1969, 40, 1001-1004. [CrossRef]

68. Weigold, E.; Hood, S.T.; Teubner, P.J.O. Energy and angular correlations of the scattered and ejected electrons in the electron-impact ionization of argon. Phys. Rev. Lett. 1973, 30, 475. [CrossRef]

69. McCarthy, I.E.; Weigold, E. (e, 2e) spectroscopy. Phys. Rep. 1976, 27, 275. [CrossRef]

70. Lohmann, B.; Weigold, E. Direct measurement of the electron momentum probability distribution in atomic hydrogen. Phys. Lett. A 1981, 86, 139-141. [CrossRef]

71. Van Wingerden, B.; Kimman, I.T.; Van Tilburg, V.T.; de Heer, F.J. Triple and double differential cross sections for electron impact ionisation of helium and molecular hydrogen. J. Phys. B 1981, 14, 2475. [CrossRef]

72. Pochat, A.; Tweed, R.J.; Doritch, M.; Peresse, J. Double and triple differential cross sections for autoionisation of helium by electron impact. J. Phys. B 1982, 15, 2269. [CrossRef]

73. Pochat, A.; Tweed, R.J.; Peresse, J.; Joachain, C.J.; Piraux, B.; Byron, F.W., Jr. Second-order effects in large-angle coplanar symmetric (e, 2e) processes. J. Phys. B 1983, 16, L775. [CrossRef]

74. Kimman, J.T.; Pan Guang-Yan, C.; McCurdy, W.; de Heer, F.J. Triple and double differential cross sections for electron impact ionisation of helium for'energy-sharing'kinematics. J. Phys. B 1983, 16, 4203. [CrossRef]

75. Cook, J.P.D.; McCarthy, I.E.; Stelbovics, A.T.; Weigold, E. Non-coplanar symmetric (e, 2e) momentum profile measurements for helium: an accurate test of helium wavefunctions. J. Phys. B 1984, 17, 2339. [CrossRef]

76. Byron, F.W., Jr.; Francken, P.; Joachain, C.J. Laser-assisted elastic electron-atom collisions. J. Phys. B 1987, 20, 5487. [CrossRef]

77. Joachain, C.H. Quantum Collision Theory, 3rd ed.; North-Holland: Amsterdam, The Netherlands, 1983.

78. Byron, F.W.; Joachain, C.J. Electron-atom collisions in a strong laser field. J. Phys. B 1984, 17, L295. [CrossRef]

79. Byron, F.W.; Joachain, C.J. Theory of (e, 2e) Reactions. Phys. Rep. 1989, 179, 211. [CrossRef]

80. Francken, P.; Attaourti, Y.; Joachain, C.J. Laser-assisted inelastic electron-atom collisions. Phys. Rev. A 1988, 38, 1785. [CrossRef]

81. Bouzidi, M.; Makhoute, A.; Khalil, D.; Maquet, A.; Joachain, C.J. On the second Born approximation for laser-assisted electron-atom collisions. J. Phys. B 2001, 34, 737. [CrossRef]

82. Bivona, S.; Burlon, R.; Zangara, R.; Ferrante, G. Electron scattering in strong laser fields. Theoretical models versus recent experiments. J. Phys. B 1985, 18, 3149. [CrossRef]

83. Francken, P.; Joachain, C.J. Theoretical study of electron-atom collisions in intense laser fields. J. Opt. Soc. Am. B 1990, 7, 554-563. [CrossRef]

84. Kroll, N.M.; Watson, K.M. Charged-particle scattering in the presence of a strong electromagnetic wave. Phys. Rev. A 1973, 8, 804. [CrossRef]

85. Ochkur, V.I. The Born-Oppenheimer method in the theory of atomic collisions. Sov. Phys. JETP 1964, $18,503-508$.

86. Nixon, K.L.; Murray, A.J. (e, 2e) ionization studies of the stable noble gases in a coplanar symmetric geometry. Phys. Rev. A 2013, 87, 022712. [CrossRef]

87. Mason, N.J. Laser-assisted electron-atom collisions. Rep. Prog. Phys. 1993, 56, 1275. [CrossRef]

88. Weigold, E.; Noble, C.J.; Hood, S.T.; Fuss, I. Electron impact ionisation of atomic hydrogen: experimental and theoretical (e, 2e) differential cross sections. J. Phys. B 1979, 12, 291. [CrossRef]

(C) 2019 by the authors. Licensee MDPI, Basel, Switzerland. This article is an open access article distributed under the terms and conditions of the Creative Commons Attribution (CC BY) license (http:/ / creativecommons.org/licenses/by/4.0/). 